BULLETIN (New Series) OF THE

AMERICAN MATHEMATICAL SOCIETY

Volume 50, Number 1, January 2013, Pages 1-55

S 0273-0979(2012)01387-3

Article electronically published on October 12, 2012

\title{
LANGLANDS PROGRAM, TRACE FORMULAS, AND THEIR GEOMETRIZATION
}

\author{
EDWARD FRENKEL \\ Notes for the AMS Colloquium Lectures \\ at the Joint Mathematics Meetings in Boston, January 4-6, 2012
}

\begin{abstract}
The Langlands Program relates Galois representations and automorphic representations of reductive algebraic groups. The trace formula is a powerful tool in the study of this connection and the Langlands Functoriality Conjecture. After giving an introduction to the Langlands Program and its geometric version, which applies to curves over finite fields and over the complex field, I give a survey of my recent joint work with Robert Langlands and Ngô Bao Châu on a new approach to proving the Functoriality Conjecture using the trace formulas, and on the geometrization of the trace formulas. In particular, I discuss the connection of the latter to the categorification of the Langlands correspondence.
\end{abstract}

\section{Contents}

1. Introduction

2. The classical Langlands Program

2.1. The case of $G L_{n}$

2.2. Examples

2.3. Function fields

2.4. The Langlands correspondence

2.5. Langlands dual group

3. The geometric Langlands correspondence

3.1. ${ }^{L} G$-bundles with flat connection

3.2. Sheaves on $\operatorname{Bun}_{G}$

3.3. Hecke functors: examples

3.4. Hecke functors: general definition

3.5. Hecke eigensheaves

3.6. Geometric Langlands correspondence

3.7. Categorical version

4. Langlands functoriality and trace formula

4.1. The Langlands Functoriality Principle

4.2. Geometric functoriality

4.3. Non-tempered representations

4.4. Trace formula

4.5. Strategy

Received by the editors February 10, 2012, and, in revised form, June 17, 2012.

2010 Mathematics Subject Classification. Primary 11R39, 14D24, 22 E57.

Supported by DARPA under the grant HR0011-09-1-0015. 
4.6. Example 26

4.7. Definition of $\mathbf{K}_{d, \rho} \quad 27$

4.8. Eigenvalues of $\mathbf{K}_{d, \rho}$

4.9. Proof of Lemma $1 \quad 28$

4.10. Decomposition of the trace formula 29

5. Geometrization of the orbital part of the trace formula 31

5.1. Geometric Arthur parameters

5.2. The sheaf $\mathcal{K}_{d, \rho}$

5.3. The functor $\mathbb{K}_{d, \rho}$

5.4. Geometrization of the orbital side 35

5.5. The moduli stack of $G$-pairs 37

5.6. Definition of the moduli stack 37

5.7. Comparison with the Hitchin moduli stack 38

5.8. Analogue of the Hitchin map for $G$-pairs 39

5.9. Example of $G L_{2}$

5.10. General case 42

6. The geometric trace formula 43

6.1. The left-hand side of the trace formula 433

6.2. Lefschetz fixed point formula interpretation 43

6.3. Geometrization of the spectral side of the trace formula 44

6.4. Connection to the Atiyah-Bott-Lefschetz fixed point formula 46

7. Relative geometric trace formula 47

7.1. Relative trace formula 48

7.2. Geometric meaning: right-hand side 50

7.3. Geometric meaning: left-hand side 51

7.4. Interpretation from the point of view of the categorical Langlands correspondence

Acknowledgments

About the author $\quad 52$

References 53

\section{INTRODUCTION}

The Langlands Program was initiated by Robert Langlands in the late 1960s in order to connect number theory and harmonic analysis [L1]. In the last 40 years a lot of progress has been made in proving the Langlands conjectures, but much more remains to be done. We still do not know the underlying reasons for the deep and mysterious connections suggested by these conjectures. But in the meantime, these ideas have propagated to other areas of mathematics, such as geometry and representation theory of infinite-dimensional Lie algebras, and even to quantum physics, bringing a host of new ideas and insights. There is hope that expanding the scope of the Langlands Program will eventually help us get the answers to the big questions about the Langlands duality.

In this lecture I will give an overview of this subject and describe my recent joint work with Robert Langlands and Ngô Bao Châu [FLN, FN] on the Functoriality Principle and the geometrization of the trace formulas. 
The key objects in the Langlands Program are automorphic representations of a reductive algebraic group $G$ over a global field $F$, which is either a number field or the field of rational functions on a smooth projective curve $X$ over a finite field. These are the constituents in the decomposition of the space $L_{2}\left(G(F) \backslash G\left(\mathbb{A}_{F}\right)\right.$ ) under the right action of $G\left(\mathbb{A}_{F}\right)$, where $\mathbb{A}_{F}$ is the ring of adèles of $F$ (see Section 2 for details). Let $G$ and $H$ be two reductive algebraic groups over $F$, and ${ }^{L} G$ and ${ }^{L} H$ their Langlands dual groups as defined in [L1]. The Langlands Functoriality Principle [L1] states that for each admissible homomorphism

$$
{ }^{L} H \rightarrow{ }^{L} G
$$

there exists a transfer of automorphic representations, from those of $H\left(\mathbb{A}_{F}\right)$ to those of $G\left(\mathbb{A}_{F}\right)$, satisfying some natural properties. Functoriality has been established in some cases, but is still unknown in general (see [Art1 for a survey).

In [FLN], following L2, L3] (see also [L4, L5]), a strategy for proving functoriality was proposed. In the space of automorphic functions on $G(F) \backslash G\left(\mathbb{A}_{F}\right)$, we construct a family of integral operators $\mathbf{K}_{d, \rho}$, where $d$ is a positive integer and $\rho$ is a finitedimensional representation of ${ }^{L} G$, which for sufficiently large $d$ project onto the automorphic representations of $G\left(\mathbb{A}_{F}\right)$ that come by functoriality from automorphic representations of certain groups $H$ determined by $\rho$. We then apply the ArthurSelberg trace formula

$$
\operatorname{Tr} \mathbf{K}_{d, \rho}=\int K_{d, \rho}(x, x) d x,
$$

where $K_{d, \rho}(x, y)$ is the kernel of $\mathbf{K}_{d, \rho}$, a function on the square of $G(F) \backslash G\left(\mathbb{A}_{F}\right)$. The left-hand, spectral, side of (1.1) may be written as the sum over irreducible automorphic representations $\pi$ of $G\left(\mathbb{A}_{F}\right)$ :

$$
\sum_{\pi} m_{\pi} \operatorname{Tr}\left(\mathbf{K}_{d, \rho}, \pi\right)
$$

where $m_{\pi}$ is the multiplicity of $\pi$ in the space of $L_{2}$ functions on $G(F) \backslash G\left(\mathbb{A}_{F}\right)$ ) (here we ignore the continuous part of the spectrum). The right-hand, orbital, side of (1.1) may be written as a sum over the conjugacy classes $\gamma$ in $G(F)$ :

$$
\sum_{\gamma \in G(F) / \text { conj. }} a_{\gamma} O_{\gamma}\left(K_{d, \rho}\right),
$$

where $O_{\gamma}\left(K_{d, \rho}\right)$ is an "orbital integral": an integral over the conjugacy class of $\gamma$ in $G\left(\mathbb{A}_{F}\right)$ (see Section 4.4).

The idea is to analyze the orbital side of the trace formula and compare the corresponding orbital integrals of the group $G$ to those of the groups $H$. This way one hopes to connect the spectral sides of the trace formulas for $G$ and $H$ and hence prove functoriality. In [FLN, FN] we related this to the geometric and categorical forms of the Langlands correspondence and made the first steps in developing the geometric methods for analyzing these orbital integrals in the case of the function field of a curve $X$ over a finite field $\mathbb{F}_{q}$.

By a geometrization of the trace formula (1.1) we understand representing each side as the trace of the Frobenius automorphism on a vector space equipped with an action of $\operatorname{Gal}\left(\overline{\mathbb{F}}_{q} / \mathbb{F}_{q}\right.$ ) (the Galois group of the finite field $\mathbb{F}_{q}$, over which our curve $X$ is defined).

Such a reformulation is useful because, first of all, unlike mere numbers, these vector spaces may well carry additional structures that could help us understand 
the connections we are looking for. For example, in B. C. Ngô's beautiful recent proof of the fundamental lemma [N2, he used the étale cohomologies of certain moduli spaces (the fibers of the so-called Hitchin map). Ngô showed that these cohomologies carry natural actions of finite groups, and he used these actions to isolate the "right" pieces of these cohomologies and to prove their isomorphisms for different groups. The equality of the traces of the Frobenius on these vector spaces then yields the fundamental lemma.

The second reason why geometrization is useful is that we expect that, unlike the corresponding numbers, these vector spaces would also make sense for curves defined over $\mathbb{C}$, so that we would obtain a version of the trace formula for complex curves.

As the first step in the program of geometrization of trace formulas, we showed in [FN that the kernels $K_{d, \rho}$ constructed in [FLN may be obtained using the Grothendieck faisceaux-fonctions dictionary from perverse sheaves $\mathcal{K}_{d, \rho}$ on a certain algebraic stack over the square of $\operatorname{Bun}_{G}$, the moduli stack of $G$-bundles on $X$. Hence the right-hand side of the trace formula (1.1) may indeed be written as the trace of the Frobenius on the étale cohomology of the restriction of $\mathcal{K}_{d, \rho}$ to the diagonal in $\operatorname{Bun}_{G} \times \operatorname{Bun}_{G}$. This may be further rewritten as the cohomology of a sheaf defined on the moduli stack of " $G$-pairs", which is closely related to the Hitchin moduli stack of Higgs bundles on $X$ (see [FN] and Section [5.6). Thus, we obtain a geometrization of the orbital side of the trace formula (1.1).

The idea then is to use the geometry of these moduli stacks to prove the desired identities of orbital integrals for $G$ and $H$ by establishing isomorphisms between the corresponding cohomologies. As we mentioned above, a geometric approach of this kind turned out to be very successful in B. C. Ngô's recent proof of the fundamental lemma N2]. The elegant argument of [N2 takes advantage of the decomposition of the cohomology of the fibers of the Hitchin map under the action of finite groups. In our case, the decomposition of the cohomology we are looking for does not seem to be due to an action of a group. Hence we have to look for other methods. Some conjectures in this direction were formulated in $[\mathrm{FN}]$; we discuss them in Section 5.10 .

The moduli stacks and the sheaves on them that appear in this picture have natural analogues for curves over $\mathbb{C}$, and hence the geometrization allows us to include complex curves into consideration. We can then use the methods of complex algebraic geometry (some of which have no obvious analogues over a finite field) to tackle the questions of functoriality that we are interested in.

Thus, the geometrization of the right-hand (orbital) side (1.3) of (1.1) is the cohomology of a sheaf on the moduli stack of $G$-pairs. In $\mathrm{FN}$ we also looked for a geometrization of the left-hand (spectral) side of (1.1), trying to interpret the sum (1.2) as the Lefschetz trace formula for the trace of the Frobenius on the étale cohomology of an $\ell$-adic sheaf. It is not obvious how to do this, because the set of the $\pi$ 's appearing in (1.2) is not the set of points of a moduli space (or stack) in any obvious way.

However, according to the Langlands correspondence [L1], reviewed in Section 2 , the $L$-packets of irreducible (tempered) automorphic representations of $G\left(\mathbb{A}_{F}\right)$ are supposed to be parametrized by the homomorphisms

$$
\sigma: W(F) \rightarrow{ }^{L} G,
$$


where $W(F)$ is the Weil group of the function field $F$ and ${ }^{L} G$ is the Langlands dual group to $G$. Therefore, assuming the Langlands correspondence, we may rewrite the sum (1.2) as a sum over such $\sigma$. Unfortunately, if $k$, the field of definition of our curve $X$, is a finite field, there is no reasonable algebraic stack whose $k$-points are the equivalence classes of homomorphisms $\sigma$. But if $k=\mathbb{C}$, such a stack exists! If we restrict ourselves to the unramified $\sigma$, then we can use the algebraic stack $\operatorname{Loc}_{L_{G}}$ of flat ${ }^{L} G$-bundles on $X$. Hence, we can pose the following question: Define a sheaf on this stack such that its cohomology (representing the left-hand side of (1.1) in the complex case) is isomorphic to the cohomology representing the right-hand side of (1.1). This isomorphism would then be a geometrization of the trace formula (1.1).

The idea of Ngô and myself [FN] is that the answer may be obtained in the framework of a categorical form of the geometric Langlands correspondence, which we review below. It is a conjectural equivalence between derived categories of $\mathcal{O}-$ modules on the moduli stack $\operatorname{Loc}_{L_{G}}$ and $\mathcal{D}$-modules on the moduli stack Bun ${ }_{G}$ of $G$-bundles on $X$. Such an equivalence has been proved in the abelian case by G. Laumon Lau2 and M. Rothstein $[\mathbf{R}$, and in the non-abelian case it has been suggested as a conjectural guiding principle by A. Beilinson and V. Drinfeld (see, e.g., [F1, VLaf, LafL] for an exposition). This categorical version of the geometric Langlands correspondence also appears naturally in the $S$-duality picture developed by A. Kapustin and E. Witten [KW] (see F2 for an exposition).

At the level of objects, the categorical Langlands correspondence assigns to the skyscraper $\mathcal{O}$-module supported at a given flat ${ }^{L} G$-bundle $\mathcal{E}$ on $X$ a Hecke eigensheaf on $\operatorname{Bun}_{G}$ with "eigenvalue" $\mathcal{E}$. But an equivalence of categories also gives us non-trivial information about morphisms; namely, the Hom's between the objects corresponding to each other on the two sides should be isomorphic. The idea of [FN], which we review below, is that for suitable objects the isomorphism of their Hom's yields the sought-after geometric trace formula. This led us to propose in [FN] a conjectural geometrization of the trace formula (1.1) in this framework, which we review in Section 6 .

In deriving this geometric trace formula, we work with the Hom's in the categories of sheaves on the squares of $\operatorname{Bun}_{G}$ and $\operatorname{Loc}_{L_{G}}$, because this is where the kernels of our functors "live".

We also obtained in [FN an analogous statement in the categories of sheaves on the stacks Bun $G$ and $\operatorname{Loc}_{L}$ themselves. The result is a geometrization of the relative trace formula, also known as the Kuznetsov trace formula, see, e.g., [J]. This formula has some favorable features compared to the usual trace formula (for example, only tempered automorphic representations, and only one representation from each $L$-packet - the "generic" one - are expected to contribute). But there is a price: in the sum (1.2) appears a weighting factor, the reciprocal of the value of the $L$-function of $\pi$ in the adjoint representation at $s=1$. The insertion of this factor in this context has been considered previously in $[\mathrm{S}]$ and $[\mathrm{Ve}$ for the group $G L_{2}$. Ngô and I showed in [FN] (see Section 6.4) that this factor also has a natural geometric interpretation, as coming from the Atiyah-Bott-Lefschetz fixed point formula.

The geometric trace formulas proposed by Ngô and myself in [FN] are still in a preliminary form, because several important issues need to be worked out. Nevertheless, we believe that they contain interesting features and even in this rough 
form might provide a useful framework for a better geometric understanding of the trace formula as well as the geometric Langlands correspondence.

These notes are organized as follows. We start with a brief introduction to the classical Langlands correspondence in Section 2 and its geometric and categorical forms in Section 3 . In Section 4 we survey the trace formula and its applications to the functoriality of automorphic representations, following FLN. In Section 5 we describe, following [FN], the geometrization of the orbital side of the trace formula in terms of the cohomology of certain sheaves on the moduli stacks which are grouplike analogues of the Hitchin moduli stacks of Higgs bundles. The geometrization of the spectral side of the trace formula is discussed in Section 6 and the relative trace formula and its geometrization in Section 7 Here we follow closely [FN].

\section{The Classical Langlands Program}

The Langlands correspondence, in its original form, manifests a deep connection between number theory and representation theory. In particular, it relates subtle number theoretic data (such as the numbers of points of a mod $p$ reduction of an elliptic curve defined by a cubic equation with integer coefficients) to more easily discernible data related to automorphic forms (such as the coefficients in the Fourier series expansion of a modular form on the upper half-plane). In this section we give an outline this correspondence.

2.1. The case of $G L_{n}$. Let $\mathbb{Q}$ be the field of rational numbers. Denote by $\overline{\mathbb{Q}}$ its algebraic closure, the field of algebraic numbers, obtained by adjoining to $\mathbb{Q}$ the roots of all polynomial equations in one variable with coefficients in $\mathbb{Q}$. The arithmetic questions about algebraic numbers may be expressed as questions about the Galois group $\operatorname{Gal}(\overline{\mathbb{Q}} / \mathbb{Q})$ of all field automorphisms of $\mathbb{Q}$.

A marvelous insight of Robert Langlands was to conjecture [L1 that there exists a connection between $n$-dimensional representations of $\mathrm{Gal}(\overline{\mathbb{Q}} / \mathbb{Q})$ and irreducible representations of the group $G L_{n}\left(\mathbb{A}_{\mathbb{Q}}\right)$ which occur in the space of $L_{2}$ functions on the quotient $G L_{n}(\mathbb{Q}) \backslash G L_{n}\left(\mathbb{A}_{\mathbb{Q}}\right)$.

Here $\mathbb{A}_{\mathbb{Q}}$ is the restricted product of all completions of $\mathbb{Q}$ : the field $\mathbb{Q}_{p}$ p-adic numbers, where $p$ runs over all primes, and the field $\mathbb{R}$ of real numbers. Elements of $\mathbb{A}_{\mathbb{Q}}$ are infinite collections

$$
\left(\left(x_{p}\right)_{p \text { prime }}, x_{\infty}\right),
$$

where for all but finitely many primes $p$ we have $x_{p} \in \mathbb{Z}_{p} \subset \mathbb{Q}_{p}$, the ring of $p$ adic integers. (This is the meaning of the word "restricted" used above.) We have a diagonal embedding $\mathbb{Q} \hookrightarrow \mathbb{A}_{\mathbb{Q}}$, and hence an embedding of groups $G L_{n}(\mathbb{Q}) \hookrightarrow$ $G L_{n}\left(\mathbb{A}_{\mathbb{Q}}\right)$.

Under the right action of $G L_{n}\left(\mathbb{A}_{\mathbb{Q}}\right)$ on $L_{2}\left(G L_{n}(\mathbb{Q}) \backslash G L_{n}\left(\mathbb{A}_{\mathbb{Q}}\right)\right)$, we have a decomposition into irreducible representations appearing both discretely and continuously:

$$
L_{2}\left(G L_{n}(\mathbb{Q}) \backslash G L_{n}\left(\mathbb{A}_{\mathbb{Q}}\right)\right)=\bigoplus \pi \bigoplus \text { continuous spectrum } .
$$

The irreducible representations $\pi$ appearing in the discrete spectrum, as well as the suitably defined constituents of the continuous spectrum (we are not going to give the precise definition) are called automorphic representations. The theory of Eisenstein series reduces the study of automorphic representations to the study of those occurring in the discrete spectrum. 
Schematically, the Langlands correspondence for $G L_{n}$ may be formulated as a correspondence between the equivalence classes of the following data.

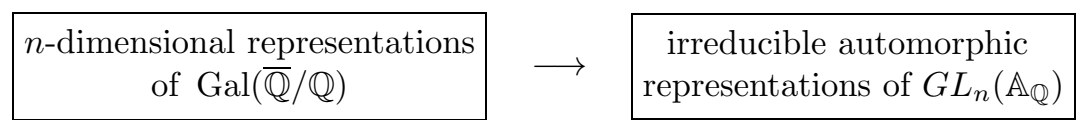

The Langlands correspondence is very useful for understanding deep questions in number theory. First of all, according to the "Tannakian philosophy", one can reconstruct a group from the category of its finite-dimensional representations, equipped with the structure of the tensor product. Describing the equivalence classes of $n$-dimensional representations of the Galois group may be viewed as a first step towards understanding its structure.

Technical point. In order to describe the tensor category of representations of $\operatorname{Gal}(\overline{\mathbb{Q}} / \mathbb{Q})$, one needs to do much more: one has to consider the categories of automorphic representations of $G L_{n}\left(\mathbb{A}_{\mathbb{Q}}\right)$ for all $n$ at once and define various functors between them corresponding to taking the direct sums and tensor products of Galois representations. This is closely related to the functoriality of automorphic representations that we discuss in Section 4 below.

Second, there are many interesting representations of Galois groups arising in "nature". Indeed, the group $\operatorname{Gal}(\overline{\mathbb{Q}} / \mathbb{Q})$ acts on the geometric invariants (such as the étale cohomologies) of an algebraic variety defined over $\mathbb{Q}$. For example, if we take an elliptic curve $E$ over $\mathbb{Q}$, then we will obtain a representation of $\operatorname{Gal}(\overline{\mathbb{Q}} / \mathbb{Q})$ on its first étale cohomology, which is a two-dimensional vector space (much like the first cohomology of an elliptic curve defined over $\mathbb{C}$ ).

Under the Langlands correspondence (2.1), some important invariants attached to Galois representations and automorphic representations have to to match. These are the so-called Frobenius eigenvalues for the former and the Hecke eigenvalues for the latter. They are attached to all but finitely many primes. We will discuss the latter in more detail in Section 3.5, and for the former, see [F1, Section 1.5].

2.2. Examples. The correspondence (2.1) is well understood for $n=1$. This is the abelian case. Indeed, one-dimensional representations of any group factor through its maximal abelian quotient. In the case of $\operatorname{Gal}(\overline{\mathbb{Q}} / \mathbb{Q})$, the maximal abelian quotient is the Galois group of the maximal abelian extension of $\mathbb{Q}$. According to the Kronecker-Weber theorem, this is the field obtained by adjoining to $\mathbb{Q}$ all roots of unity. One derives from this description that the corresponding Galois group is isomorphic to the group of connected components of

$$
G L_{1}(\mathbb{Q}) \backslash G L_{1}\left(\mathbb{A}_{\mathbb{Q}}\right)=\mathbb{Q}^{\times} \backslash \mathbb{A}_{\mathbb{Q}}^{\times}
$$

This is what (2.1) boils down to for $n=1$. The Abelian Class Field Theory gives a similar adelic description of the maximal abelian quotient of the Galois group $\operatorname{Gal}(\bar{F} / F)$, where $F$ is a general number field (see, e.g., [F1, Section 1.2, for more details).

Suppose next that $n=2$. Let $\sigma$ come from the first étale cohomology of the smooth elliptic curve $E$ defined by the equation

$$
y^{2}=x^{3}+a x+b
$$

where $a, b \in \mathbb{Z}$ are such that the discriminant is non-zero, $4 a^{3}+27 b^{2} \neq 0$. The representation $\sigma$ contains a lot of important information about the curve $E$. The 
corresponding Frobenius eigenvalues encode, for each prime $p$ not dividing the discriminant, the number of points of the reduction of $E$ modulo $p, \# E\left(\mathbb{F}_{p}\right)$. This is simply the number of solutions of the equation (2.2) $\bmod p$ plus one, corresponding to the point at infinity (our $E$ is a projective curve).

According to the Langlands correspondence, $\sigma$ should correspond to a cuspidal automorphic representation $\pi$ of $G L_{2}\left(\mathbb{A}_{\mathbb{Q}}\right)$. To make things more concrete, we assign to this automorphic representation in a standard way (see, e.g., F1, Section 1.6) a modular cusp form

$$
f(q)=\sum_{n=1}^{\infty} a_{n} q^{n}
$$

on the upper half-plane $\{\tau \in \mathbb{C} \mid \operatorname{Im} \tau>0\}$, where $q=e^{2 \pi i \tau}$. The matching of the Frobenius and Hecke eigenvalues under the Langlands correspondence now becomes the statement of the Shimura-Taniyama-Weil conjecture (now a theorem [W, TW, BCDT] ): for each $E$ as above there exist a modular cusp form $f_{E}(q)$ with $a_{1}=1$ and

$$
a_{p}=p+1-\# E\left(\mathbb{F}_{p}\right)
$$

for all primes $p$ not dividing the discriminant of $E$ (and also, $a_{m n}=a_{m} a_{n}$ for all relatively prime $m$ and $n$ ).

This is a stunning result: the modular form $f_{E}$ serves as a generating function of the numbers of points of $E \bmod p$ for almost all $p$.

It implies, according to a result of K. Ribet, Fermat's Last Theorem.

One obtains similar statements by analyzing from the point of view of the Langlands correspondence the Galois representations coming from other algebraic varieties, or more general motives. This shows the great power of the Langlands correspondence: it translates difficult questions in number theory to questions in harmonic analysis.

2.3. Function fields. The correspondence (2.1) is not a bijection. But it becomes a bijection if we replace $\mathbb{Q}$ (or a more general number field) in (2.1) by a function field.

Let $X$ be a smooth projective connected curve over a finite field $k=\mathbb{F}_{q}$. The field $\mathbb{F}_{q}(X)$ of $\left(\mathbb{F}_{q}\right.$-valued) rational functions on $X$ is called the function field of $X$.

For example, suppose that $X=\mathbb{P}^{1}$. Then $\mathbb{F}_{q}(X)$ is just the field of rational functions in one variable. Its elements are fractions $P(t) / Q(t)$, where $P(t)$ and $Q(t) \neq 0$ are polynomials over $\mathbb{F}_{q}$ without common factors, with their usual operations of addition and multiplication.

It turns out that there are many similarities between function fields and number fields. For example, let's look at the completions of the function field $\mathbb{F}_{q}\left(\mathbb{P}^{1}\right)$. Consider the field $\mathbb{F}_{q}((t))$ of formal Laurent power series in the variable $t$. An element of this completion is a series of the form $\sum_{n \geq N} a_{n} t^{n}$, where $N \in \mathbb{Z}$ and each $a_{n}$ is an element of $\mathbb{F}_{q}$. Elements of $\mathbb{F}_{q}\left(\mathbb{P}^{1}\right)$ are rational functions $P(t) / Q(t)$, and such a rational function can be expanded in an obvious way in a formal power series in $t$. This defines an embedding of fields $\mathbb{F}_{q}\left(\mathbb{P}^{1}\right) \hookrightarrow \mathbb{F}_{q}((t))$, which makes $\mathbb{F}_{q}((t))$ into a completion of $\mathbb{F}_{q}\left(\mathbb{P}^{1}\right)$, with respect to a standard norm.

Observe that the field $\mathbb{F}_{p}((t))$ looks very much like the field $\mathbb{Q}_{p}$ of $p$-adic numbers. Likewise, the field $\mathbb{F}_{q}((t))$, where $q=p^{n}$, looks like a degree $n$ extension of $\mathbb{Q}_{p}$. 
The completion $\mathbb{F}_{p}((t))$ corresponds to the maximal ideal in the ring $\mathbb{F}_{q}[t]$ generated by $A(t)=t$ (note that $\mathbb{F}_{q}[t] \subset \mathbb{F}_{q}\left(\mathbb{P}^{1}\right)$ may be thought of as the analogue of $\mathbb{Z} \subset \mathbb{Q})$. Other completions of $\mathbb{F}_{q}\left(\mathbb{P}^{1}\right)$ correspond to other maximal ideals in $\mathbb{F}_{q}[t]$, which are generated by irreducible monic polynomials $A(t)$. (Those are the analogues of the ideals $(p)$ in $\mathbb{Z}$ generated by the prime numbers $p$.) There is also a completion corresponding to the point $\infty \in \mathbb{P}^{1}$, which is isomorphic to $\mathbb{F}_{q}\left(\left(t^{-1}\right)\right)$.

If the polynomial $A(t)$ has degree $m$, then the corresponding residue field is isomorphic to $\mathbb{F}_{q^{m}}$, and the corresponding completion is isomorphic to $\mathbb{F}_{q^{m}}((\widetilde{t}))$, where $\widetilde{t}$ is the uniformizer, $\tilde{t}=A(t)$. One can think of $\widetilde{t}$ as the local coordinate near the $\mathbb{F}_{q^{m}}$-point corresponding to $A(t)$, just like $t-a$ is the local coordinate near the $\mathbb{F}_{q}$-point $a$ of $\mathbb{A}^{1}$. The difference with the number field case is that all of these completions are non-archimedian; there are no analogues of the archimedian completions $\mathbb{R}$ or $\mathbb{C}$ that we have in the case of number fields.

For a general curve $X$, completions of $\mathbb{F}_{q}(X)$ are also labeled by its closed points (forming the set denoted by $|X|$ ), and the completion corresponding to a point $x$ with residue field $\mathbb{F}_{q^{n}}$ is isomorphic to $\mathbb{F}_{q^{n}}\left(\left(t_{x}\right)\right)$, where $t_{x}$ is the local coordinate near $x$ on $X$. The subring $\mathbb{F}_{q^{m}}\left[\left[t_{x}\right]\right]$ consisting of the formal Taylor series (no negative powers of $t_{x}$ ) will be denoted by $\mathcal{O}_{x}$.

2.4. The Langlands correspondence. Let $F$ be the function field of a curve $X$ over $\mathbb{F}_{q}$. The ring $\mathbb{A}_{F}$ of adèles of $F$ is by definition the restricted product of the fields $F_{x}$, where $x$ runs over the set of all closed points of $X$ :

$$
\mathbb{A}_{F}=\prod_{x \in|X|}^{\prime} F_{x} .
$$

The word "restricted" (reflected by the prime in the above product) means that we consider only the collections $\left(f_{x}\right)_{x \in X}$ of elements of $F_{x}$ in which $f_{x} \in \mathcal{O}_{x}$ for all but finitely many $x$. The ring $\mathbb{A}_{F}$ contains the field $F$, which is embedded into $\mathbb{A}_{F}$ diagonally, by taking the expansions of rational functions on $X$ at all points. One defines automorphic representations of $G L_{n}\left(\mathbb{A}_{F}\right)$ as constituents of the space of $L_{2}$ functions on the quotient $G L_{n}(F) \backslash G L_{n}\left(\mathbb{A}_{F}\right)$ defined as in the number field case.

The objects that will appear on the right-hand side of the Langlands correspondence are the so-called tempered automorphic representations. These are the representations for which the Ramanujan hypothesis is expected to hold (hence they are sometimes called Ramanujan representations). This means that each of the conjugacy classes $\nu_{x}$ in the complex group $G L_{n}$ (or ${ }^{L} G$ in general) encoding the Hecke eigenvalues of $\pi$ (see Section 3.5 below) is unitary. For $G=G L_{n}$ it is known that all cuspidal automorphic representations are tempered.

Now let $\bar{F}$ be the separable closure of $F$. We have the Galois group $\operatorname{Gal}(\bar{F} / F)$ and a natural homomorphism

$$
\operatorname{Gal}\left((\bar{F} / F) \rightarrow \operatorname{Gal}\left(\overline{\mathbb{F}}_{q} / \mathbb{F}_{q}\right),\right.
$$

due to the fact that $\mathbb{F}_{q}$ is the subfield of scalars in $F$. Now, Gal $\left(\overline{\mathbb{F}}_{q} / \mathbb{F}_{q}\right)$ is isomorphic to the pro-finite completion of $\mathbb{Z}$,

$$
\widehat{\mathbb{Z}}=\lim _{\longleftarrow} \mathbb{Z} / N \mathbb{Z}=\lim _{\longleftarrow} \operatorname{Gal}\left(\mathbb{F}_{q^{N}} / \mathbb{F}_{q}\right) .
$$

The preimage of $\mathbb{Z} \subset \widehat{\mathbb{Z}}$ in $\operatorname{Gal}((\bar{F} / F)$ under the homomorphism (2.4) is called the Weil group of $F$ and is denoted by $W(F)$. 
On the left-hand side of the Langlands correspondence we take the equivalence classes $n$-dimensional representations of the Weil group $W(F)$.

The function field analogue of the Langlands correspondence (2.1) is then given by the following diagram.

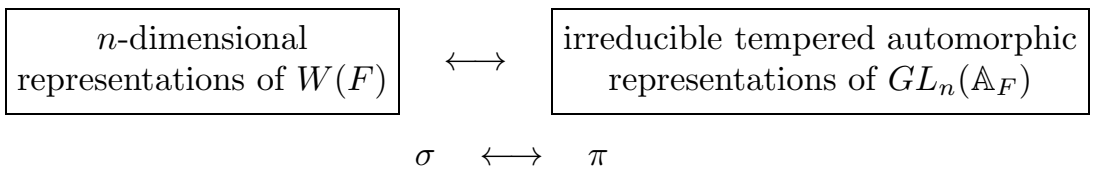

Under the correspondence the Frobenius eigenvalues of $\sigma$ should match the Hecke eigenvalues of $\pi$.

This correspondence has been proved by V. Drinfeld [D1, D2] for $n=2$ and by L. Lafforgue [LLaf] for $n>2$.

Technical point. The representations of $W(F)$ appearing on the left-hand side of (2.5) should be continuous with respect to the Krull topology on $W(F)$. However, continuous complex representations necessarily factor through a finite group. In order to obtain a large enough class of such representations, we should consider the so-called $\ell$-adic representations, defined not over $\mathbb{C}$, but over a finite extension of the field $\mathbb{Q}_{\ell}$ of $\ell$-adic numbers, where $\ell$ is a prime that does not divide $q$. Frobenius eigenvalues will then be $\ell$-adic numbers, and to match them with the Hecke eigenvalues, which are complex numbers, we need to choose once and for all an isomorphism of $\mathbb{C}$ and $\overline{\mathbb{Q}}_{\ell}$ as abstract fields (extending the identification of their subfield $\overline{\mathbb{Q}}$ ). But it follows from the theorem of Drinfeld and Lafforgue that actually they all belong to $\overline{\mathbb{Q}}$, so this isomorphism is never used, and the left-hand side of (2.5) is independent of $\ell$. For more on this, see, e.g., [F1, Section 2.2].

2.5. Langlands dual group. An important insight of Langlands L1 was that the correspondence (2.5) may be generalized by replacing the group $G L_{n}$ on the right-hand side by an arbitrary connected reductive algebraic group $G$ over the field $F$. This necessitates introducing the so-called Langlands dual group.

The simplest case to consider is that of a reductive group $G$ that is defined over the finite field of scalars $k=\mathbb{F}_{q}$ and is split over $k$. This means that it contains a maximal torus that is split (isomorphic to the direct product of copies of the multiplicative group) over $k$. In these notes we will consider slightly more general groups of the following kind: $G$ is a group scheme over $X$ which contains a Borel subgroup scheme $B$ also defined over $X$, and there exists an étale cover $X^{\prime} \rightarrow X$ such that the pull-back of $G$ to $X^{\prime}$ (resp., $B$ ) is isomorphic to the constant group scheme $X^{\prime} \times \mathbb{G}$, where $\mathbb{G}$ is a split reductive group over $k$ (resp., $X^{\prime} \times \mathbb{B}$, where $\mathbb{B}$ is a Borel subgroup of $\mathbb{G}$ ).

For example, let $\mathbb{G}$ be the multiplicative group $\mathbb{G}_{m}=G L_{1}$. Let $X^{\prime} \rightarrow X$ be an étale double cover of $X$. The group $\mathbb{Z}_{2}=\{ \pm 1\}$ acts fiberwise on $X^{\prime}$ and on $\mathbb{G}_{m}$ by the formula $x \rightarrow x^{ \pm 1}$. Define $H$ as the following group scheme over $X$ :

$$
H=X^{\prime} \underset{\mathbb{Z}_{2}}{\times} \mathbb{G}_{m}
$$

This is an example of a twisted torus. Its pull-back to $X^{\prime}$ is isomorphic to $X^{\prime} \times \mathbb{G}_{m}$. We can now talk about the group over any subscheme of $X$. For example, we have the group $H(F)$ of sections of $H$ over the generic point of $X$, Spec $F$, the local group for each $x \in|X|$, which is the group of sections of $H$ over the formal punctured disc 
$D_{x}^{\times}=\operatorname{Spec} F_{x}$, its subgroup of sections over the formal disc $D_{x}=\operatorname{Spec} \mathcal{O}_{x}$. The adelic group is now the restricted product of the local groups for all $x \in|X|$, etc.

Likewise, in general we have a group scheme

$$
G=\underset{\Gamma}{X^{\prime}} \underset{\Gamma}{\mathbb{G}},
$$

where $\Gamma$ is the group of deck transformations of $X^{\prime}$, acting on $\mathbb{G}$.

Let $\mathbb{T}$ be a maximal split torus in $\mathbb{G}$. We associate to it two lattices: the weight lattice $X^{*}(\mathbb{T})$ of homomorphisms $\mathbb{T} \rightarrow \mathbb{G}_{m}$ and the coweight lattice $X_{*}(\mathbb{T})$ of homomorphisms $\mathbb{G}_{m} \rightarrow \mathbb{T}$. They contain the sets of roots $\Delta \subset X^{*}(\mathbb{T})$ and coroots $\Delta^{\vee} \subset X_{*}(\mathbb{T})$ of $G$, respectively. The quadruple $\left(X^{*}(\mathbb{T}), X_{*}(\mathbb{T}), \Delta, \Delta^{\vee}\right)$ is called the root data for $\mathbb{G}$. It determines the split group $\mathbb{G}$ up to an isomorphism.

The action of the group $\Gamma$ on $\mathbb{G}$ gives rise to its action on the root data.

Let us now exchange the lattices of weights and coweights and the sets of roots and coroots. Then we obtain the root data

$$
\left(X_{*}(\mathbb{T}), X^{*}(\mathbb{T}), \Delta^{\vee}, \Delta\right)
$$

of another reductive algebraic group over $\mathbb{C}$ (or any other algebraically closed field, like $\overline{\mathbb{Q}}_{\ell}$ ) which is denoted by $\overleftarrow{\mathbb{G}}$. The action of $\Gamma$ on the root data gives rise to its action on $\check{G}$. We then define the Langlands dual group of $G$ as

$$
{ }^{L} G=\Gamma \ltimes \check{G} .
$$

Note that $\Gamma$ is a finite quotient of the Galois group $\operatorname{Gal}(\bar{F} / F)$.

For example, if $G$ is a twisted torus described above, then ${ }^{L} G=\mathbb{Z}_{2} \ltimes \mathbb{G}_{m}$, where $\mathbb{Z}_{2}$ acts on $\mathbb{G}_{m}$ by the formula $x \mapsto x^{ \pm 1}$.

There is a variant of the above definition in which $\Gamma$ is replaced by $\operatorname{Gal}(\bar{F} / F)$ (acting on the right factor through the surjective homomorphism $\operatorname{Gal}(\bar{F} / F) \rightarrow \Gamma$ ) or by the Weil group $W(F)$. The definition may be generalized to an arbitrary reductive group $G$ over $F$.

Now the conjectural Langlands correspondence (2.5) takes the following form.

$$
\begin{array}{|c|c|c|}
\text { homomorphisms } \\
W(F) \rightarrow{ }^{L} G
\end{array} \longleftrightarrow \begin{gathered}
\text { irreducible tempered automorphic } \\
\text { representations of } G\left(\mathbb{A}_{F}\right)
\end{gathered}
$$

Note that if $G=G L_{n}$, then ${ }^{L} G$ is also $G L_{n}$, and so the homomorphisms $W(F) \rightarrow$ ${ }^{L} G$ appearing on the left-hand side are the same as $n$-dimensional representations of $W(F)$.

Technical point. We should consider here $\ell$-adic homomorphisms, as in the case of $G L_{n}$. Also, to a homomorphism $W(F) \rightarrow{ }^{L} G$ in general corresponds not a single irreducible automorphic representation of $G\left(\mathbb{A}_{F}\right)$, but a set of such representations, called an $L$-packet.

Under the correspondence (2.6), the same kind of compatibility between the Hecke and Frobenius eigenvalues should hold as in the case of $G L_{n}$. The key point here (which comes from Langlands' interpretation [L1] of the description of the spherical Hecke algebra for general reductive groups due to Satake) is that the Hecke eigenvalues of automorphic representations may be interpreted as conjugacy classes in the Langlands dual group ${ }^{L} G$ (see Section 3.5 below and [F1, Section 5.2], for more details).

For $G=G L_{n}$ the Frobenius eigenvalues completely determine $\sigma$ and the Hecke eigenvalues completely determine an irreducible automorphic representation $\pi$. All 
automorphic representations occur with multiplicity 1 in $L_{2}\left(G(F) \backslash G\left(\mathbb{A}_{F}\right)\right)$. For general groups, this is not so. There may be several inequivalent homomorphisms $\sigma: W(F) \rightarrow{ }^{L} G$ (with the same collection of Frobenius eigenvalues), all corresponding to the same $\pi$ (or the same $L$-packet). In this case the multiplicity of $\pi$ is expected to be greater than 1 .

\section{The GeOMEtRic LAnglands CORRESPOndence}

Now we wish to reformulate the Langlands correspondence in such a way that it would make sense not only for curves defined over a finite field, but also for curves over the complex field.

Thus, we need to find geometric analogues of the notions of Galois representations and automorphic representations.

3.1. ${ }^{L} G$-bundles with flat connection. The former is fairly easy. Let $X$ be a curve over a field $k$ and $F=k(X)$ the field of rational functions on $X$. If $Y \rightarrow X$ is a covering of $X$, then the field $k(Y)$ of rational functions on $Y$ is an extension of the field $F=k(X)$ of rational functions on $X$, and the Galois group $\operatorname{Gal}(k(Y) / k(X))$ may be viewed as the group of deck transformations of the cover. If our cover is unramified, then this group is isomorphic to a quotient of the (arithmetic) fundamental group of $X$. For a cover ramified at points $x_{1}, \ldots, x_{n}$, it is isomorphic to a quotient of the (arithmetic) fundamental group of $X \backslash\left\{x_{1}, \ldots, x_{n}\right\}$. From now on we will focus on the unramified case. This means that we replace $\operatorname{Gal}(\bar{F} / F)$ by its maximal unramified quotient, which is isomorphic to the (arithmetic) fundamental group of $X$. Its geometric analogue, for $X$ defined over $\mathbb{C}$, is $\pi_{1}(X, x)$, with respect to a reference point $x \in X$.

The choice of a reference point could be a subtle issue in general. However, since these groups are isomorphic to each other for different choices of the reference point, we obtain canonical bijections between the sets of equivalence classes of homomorpisms from these groups to ${ }^{L} G$, which is what we are interested in here. Henceforth we will suppress the reference point in our notation and write simply $\pi_{1}(X)$.

Thus, the geometric counterpart of a (unramified) homomorphism $\operatorname{Gal}(\bar{F} / F) \rightarrow$ ${ }^{L} G$ is a homomorphism $\pi_{1}(X) \rightarrow{ }^{L} G$. If we replace $\operatorname{Gal}(\bar{F} / F)$ by the Weil group $W(F)$, then we should replace $\pi_{1}(X)$ by a similarly defined subgroup.

Let $X$ be a smooth projective connected algebraic curve defined over $\mathbb{C}$. Let $G$ be a complex reductive algebraic group and ${ }^{L} G$ its Langlands dual group. Then homomorphisms $\pi_{1}(X) \rightarrow{ }^{L} G$ may be described in differential geometric terms as (smooth) principal ${ }^{L} G$-bundles on $X$ with a flat connection. Indeed, the monodromy of the flat connection gives rise to a homomorphism $\pi_{1}(X) \rightarrow{ }^{L} G$, and this gives rise to an equivalence of the appropriate categories and a bijection of the corresponding sets of equivalence classes.

Let $E$ be a smooth principal ${ }^{L} G$-bundle on $X$. A flat connection on $E$ has two components. The $(0,1)$ component, with respect to the complex structure on $X$, defines holomorphic structure on $E$, and the $(1,0)$ component defines a holomorphic connection $\nabla$. Thus, a principal ${ }^{L} G$-bundle with a flat connection on $X$ is the same as a pair $(E, \nabla)$, where $E$ is a holomorphic (equivalently, algebraic) principal ${ }^{L} G$ bundle on $X$ and $\nabla$ is a holomorphic (equivalently, algebraic) connection on $E$. 
Thus, for complex curves the objects on the left-hand side of the Langlands correspondence (2.6) should be the equivalence classes of flat (holomorphic or algebraic) ${ }^{L} G$-bundles $(E, \nabla)$.

3.2. Sheaves on $\operatorname{Bun}_{G}$. We consider next the right-hand side of (2.6). Here the answer is not quite as obvious. We sketch it briefly referring the reader to [F1, Section 3], for more details.

Recall that automorphic representations of $G\left(\mathbb{A}_{F}\right)$ (where $F$ is a function field of a curve $X$ defined over $\mathbb{F}_{q}$ ) are realized in functions on the quotient $G(F) \backslash G\left(\mathbb{A}_{F}\right)$. Let us restrict ourselves to those irreducible automorphic representations that correspond to unramified homomorphisms $W(F) \rightarrow{ }^{L} G$. It is known that they contain a one-dimensional subspace stable under the subgroup $G\left(\mathcal{O}_{F}\right) \subset G\left(\mathbb{A}_{F}\right)$, where

$$
\mathcal{O}_{F}=\prod_{x \in|X|} \mathcal{O}_{x}
$$

These representations are also called unramified. Any vector in the $G\left(\mathcal{O}_{F}\right)$-stable line in such a representation $\pi$ gives rise to a function on the double quotient

$$
G(F) \backslash G\left(\mathbb{A}_{F}\right) / G\left(\mathcal{O}_{F}\right) .
$$

This function, which is called the spherical function, contains all information about $\pi$, because the right translates by $g \in G\left(\mathbb{A}_{F}\right)$ of this function pulled back to $G(F) \backslash G\left(\mathbb{A}_{F}\right)$ span $\pi$.

Now, a key observation, due to André Weil, is that in the case of $G=G L_{n}$ this double quotient is precisely the set of isomorphism classes of rank $n$ bundles on our curve $X$. This statement is true if the curve $X$ is defined over a finite field or the complex field.

For a general reductive group $G$ this double quotient is the set of isomorphism classes of principal $G$-bundles on $X$ if $X$ is over $\mathbb{C}$. This is true in Zariski, étale, or analytic topology.

If $X$ is defined over a finite field, the situation is more subtle 1 Then the double quotient (3.1) is the set of equivalence classes of principal $G$-bundles in Zariski topology as well as Nisnevich topology [Ni1, Ni2]. In the étale topology, this is true only if the group

$$
\operatorname{Ker}^{1}(F, G)=\operatorname{Ker}\left(H^{1}(F, G) \rightarrow \prod_{x \in|X|} H^{1}\left(F_{x}, G\right)\right)
$$

is trivial. In this case, it is sometimes said that $G$ "satisfies the Hasse principle". This holds, for example, in the case that $G$ is semi-simple and split over $\mathbb{F}_{q}$; see [Ha] and $[\mathrm{BeDh}$, Corollary 4.2]. Otherwise, the set of equivalence classes of principal $G$-bundles in the étale topology (equivalently, the $f p p f$ topology) is a union over $\xi \in \operatorname{Ker}^{1}(F, G)$ of double quotients like (3.1) in which $G(F)$ is replaced by its form corresponding to $\xi$.

From now on we will assume for simplicity that the Hasse principle holds for $G$. Then the geometric analogues of unramified automorphic representations should be some geometric objects that "live" on some kind of moduli space of principal $G$-bundles on $X$.

\footnotetext{
1 I thank Yevsey Nisnevich for a discussion of this issue.
} 
If $G=G L_{1}$, the Picard variety is an algebraic variety that serves as the moduli space of principal $G$-bundles on $X$, which are the same as line bundles on $X$ in this case.

Unfortunately, for a non-abelian group $G$ there is no algebraic variety whose set of $k$-points is the set of isomorphism classes of principal $G$-bundles on $X$ (where $k$ is the field of definition of $X$ ). The reason is that $G$-bundles have groups of automorphisms, which vary from bundle to bundle (in the case of $G L_{1}$-bundles, the group of automorphisms is the same for all bundles; it is the multiplicative group acting by rescalings). However, there is an algebraic stack that parametrizes principal $G$-bundles on $X$. It is denoted by $\operatorname{Bun}_{G}$. It is not an algebraic variety, but it looks locally like the quotient of an algebraic variety by the action of an algebraic group. These actions are not free, and therefore the quotient is no longer an algebraic variety. However, the structure of the quotient allows us to define familiar objects on it. For instance, a coherent sheaf on the quotient stack $Y / H$ of this kind is nothing but an $H$-equivariant coherent sheaf on $Y$. It turns out that this is good enough for our purposes.

In the classical story, when $X$ is defined over $\mathbb{F}_{q}$, an unramified automorphic representation may be replaced by a non-zero spherical function (which is unique up to a scalar) on the above double quotient which is the set of $\mathbb{F}_{q}$-points of $\operatorname{Bun}_{G}$. Hence in the geometric theory we need to find geometric analogues of these functions.

According to Grothendieck's philosophy, the "correct" geometric counterpart of the notion of a function on the set of $\mathbb{F}_{q}$-points of $V$ is the notion of an $\ell$-adic sheaf on $V$. We will not attempt to give a precise definition here, referring the reader to [M, FK]. Let us just say that the simplest example of an $\ell$-adic sheaf is an $\ell$-adic local system, which is, roughly speaking, a compatible system of locally constant $\mathbb{Z} / \ell^{n} \mathbb{Z}$-sheaves on $V$ for $n \geq 1$ (in the étale topology).

The important property of the notion of an $\ell$-adic sheaf $\mathcal{F}$ on $V$ is that for any morphism $f: V^{\prime} \rightarrow V$ from another variety $V^{\prime}$ to $V$ the group of symmetries of this morphism will act on the pull-back of $\mathcal{F}$ to $V^{\prime}$. In particular, let $x$ be an $\mathbb{F}_{q}$-point of $V$ and $\bar{x}$ the $\overline{\mathbb{F}}_{q}$-point corresponding to an inclusion $\mathbb{F}_{q} \hookrightarrow \overline{\mathbb{F}}_{q}$. Then the pull-back of $\mathcal{F}$ with respect to the composition $\bar{x} \rightarrow x \rightarrow V$ is a sheaf on $\bar{x}$, which is nothing but the fiber $\mathcal{F}_{\bar{x}}$ of $\mathcal{F}$ at $\bar{x}$, which is a $\overline{\mathbb{Q}}_{\ell}$-vector space. But the Galois group $\operatorname{Gal}\left(\overline{\mathbb{F}}_{q} / \mathbb{F}_{q}\right)$ is the symmetry of the map $\bar{x} \rightarrow x$, and therefore it acts on $\mathcal{F}_{\bar{x}}$.

Let $\operatorname{Fr}_{\bar{x}}$ be the (geometric) Frobenius element, which is the inverse of the automorphism $y \mapsto y^{q}$ of $\overline{\mathbb{F}}_{q}$. It is a generator of $\operatorname{Gal}\left(\overline{\mathbb{F}}_{q} / \mathbb{F}_{q}\right)$ and hence acts on $\mathcal{F}_{\bar{x}}$. Taking the trace of $\operatorname{Fr}_{\bar{x}}$ on $\mathcal{F}_{\bar{x}}$, we obtain a number $\operatorname{Tr}\left(\operatorname{Fr}_{\bar{x}}, \mathcal{F}_{\bar{x}}\right)$, which we will also denote by $\operatorname{Tr}\left(\operatorname{Fr}_{x}, \mathcal{F}_{x}\right)$.

Hence we obtain a function on the set of $\mathbb{F}_{q}$-points of $V$. One assigns similarly a function to a complex of $\ell$-adic sheaves, by taking the alternating sums of the traces of $\mathrm{Fr}_{\bar{x}}$ on the stalk cohomologies of $\mathbb{K}$ at $\bar{x}$. The resulting map intertwines the natural operations on complexes of sheaves with natural operations on functions (see [Lau1, Section 1.2]). For example, pull-back of a sheaf corresponds to the pullback of a function, and push-forward of a sheaf with compact support corresponds to the fiberwise integration of a function.

Thus, because of the existence of the Frobenius automorphism in the Galois group $\operatorname{Gal}\left(\overline{\mathbb{F}}_{q} / \mathbb{F}_{q}\right)$ (which is the group of symmetries of an $\mathbb{F}_{q}$-point), we can pass from $\ell$-adic sheaves to functions on any algebraic variety over $\mathbb{F}_{q}$. This suggests 
that the proper geometrization of the notion of a function in this setting is the notion of $\ell$-adic sheaf.

The naive abelian category of $\ell$-adic sheaves is not a good choice for various reasons; for instance, it is not stable under the Verdier duality. The correct choice turns out to be another abelian category of the so-called perverse sheaves. These are actually complexes of $\ell$-adic sheaves on $V$ satisfying certain special properties. Examples are $\ell$-adic local systems on a smooth variety $V$, placed in cohomological degree equal to $-\operatorname{dim} V$. General perverse sheaves are glued from such local systems defined on the strata of a particular stratification of $V$ by locally closed subvarieties.

Experience shows that many "interesting" functions on the set $V\left(\mathbb{F}_{q}\right)$ of points of an algebraic variety $V$ over $\mathbb{F}_{q}$ come from perverse sheaves $\mathcal{K}$ on $V$. Hence it is natural to expect that unramified automorphic functions on

$$
G(F) \backslash G\left(\mathbb{A}_{F}\right) / G\left(\mathcal{O}_{F}\right),
$$

which is the set of $\mathbb{F}_{q}$-points of $\operatorname{Bun}_{G}$, come from perverse sheaves on $\operatorname{Bun}_{G}$.

The concept of perverse sheaves makes perfect sense for varieties over $\mathbb{C}$ as well, and this allows us to formulate the geometric Langlands conjecture when $X$ (and hence $\operatorname{Bun}_{n}$ ) is defined over $\mathbb{C}$. And over the field of complex numbers there is one more reformulation that we can make; namely, we can pass from perverse sheaves to $\mathcal{D}$-modules.

Recall (see, e.g., $\mathrm{KS}, \mathrm{GM}$ ) that a $\mathcal{D}$-module on a smooth algebraic variety $Z$ is a sheaf of modules over the sheaf $\mathcal{D}_{Z}$ of differential operators on $Z$. An example of a $\mathcal{D}$-module is the sheaf of sections of a flat vector bundle on $Z$. The sheaf of functions on $Z$ acts on sections by multiplication, so it is an $\mathcal{O}_{Z}$-module. But the flat connection also allows us to act on sections by vector fields on $Z$. This gives rise to an action of the sheaf $\mathcal{D}_{Z}$, because it is generated by vector fields and functions. Thus, we obtain the structure of a $\mathcal{D}$-module.

In our case, Bun $_{G}$ is not a variety, but an algebraic stack. The suitable (derived) category of $\mathcal{D}$-modules on it has been defined in $\mathrm{BD}$.

$\mathcal{D}$-modules on $\operatorname{Bun}_{G}$ will be the objects that we will consider as the replacements for the unramified spherical functions in the complex case.

3.3. Hecke functors: examples. There is more: an unramified spherical function attached to an unramified automorphic representation has a special property; it is an eigenfunction of the Hecke operators. These are integral operators that are cousins of the classical Hecke operators one studies in the theory of modular forms. The eigenvalues of these operators are precisely what we referred to earlier as Hecke eigenvalues. For a general automorphic representation, these are defined for all but finitely many closed points of $X$. But for the unramified automorphic representations they are defined for all points. In this case the Hecke operators may be defined as integral operators acting on the space of functions on the set of $\mathbb{F}_{q}$-points of $\operatorname{Bun}_{G}$, if the curve $X$ is defined over $\mathbb{F}_{q}$.

The $\mathcal{D}$-modules on $\operatorname{Bun}_{G}$ we are looking for, in the case that $X$ is defined over $\mathbb{C}$, should reflect this Hecke property.

The analogues of the Hecke operators are now the so-called Hecke functors acting on the derived category of $\mathcal{D}$-modules on $\operatorname{Bun}_{G}$. They are labeled by pairs $(x, V)$, where $x \in X$ and $V$ is a finite-dimensional representation of the dual group ${ }^{L} G$, and are defined using certain modifications of $G$-bundles. 
Before giving the general definition, consider two examples. First, consider the abelian case with $G=G L_{1}$ (thus, we have $G(\mathbb{C})=\mathbb{C}^{\times}$). In this case $\operatorname{Bun}_{G}$ may be replaced by the Picard variety $P i c$ which parametrizes line bundles on $X$. Given a point $x \in X$, consider the map $p_{x}^{\prime}:$ Pic $\rightarrow$ Pic sending a line bundle $\mathcal{L}$ to $\mathcal{L}(x)$ (the line bundle whose sections are sections of $\mathcal{L}$ which are allowed to have a pole of order 1 at $x$ ). By definition, the Hecke functor $\mathbb{H}_{1, x}$ corresponding to $x$ and $1 \in \mathbb{Z}$ (which we identify with the set of one-dimensional representations of ${ }^{L} G=G L_{1}$ ) is given by the formula

$$
\mathbb{H}_{1, x}(\mathcal{F})=p_{x}^{\prime *}(\mathcal{F})
$$

Next, consider the case of $G=G L_{n}$ and $V=V_{\check{\omega}_{1}}$, the defining $n$-dimensional representation of ${ }^{L} G=G L_{n}$. In this case $\operatorname{Bun}_{G L_{n}}$ is the moduli stack $\operatorname{Bun}_{n}$ of rank $n$ bundles on $X$. There is an obvious analogue of the map sending a rank $n$ bundle $\mathcal{M}$ to $\mathcal{M}(x)$. But then the degree of the bundle jumps by $n$. It is possible to increase it by 1 , but we need to choose a line $\ell$ in the fiber of $\mathcal{M}$ at $x$. We then define a new rank $n$ bundle $\mathcal{M}^{\prime}$ by saying that its sections are the sections of $\mathcal{M}$ having a pole of order 1 at $x$, but the polar part has to belong to $\ell$. Then $\operatorname{deg} \mathcal{M}^{\prime}=\operatorname{deg} \mathcal{M}+1$. However, we now have a $\mathbb{P}^{n-1}$ worth of modifications of $\mathcal{M}$ corresponding to different choices of the line $\ell$. The Hecke functor $\mathbb{H}_{V_{\tilde{\omega}_{1}, x}}$ is obtained by integrating over all of them.

More precisely, let $\mathcal{H}_{\check{\omega}_{1}, x}$ be the moduli stack of pairs $\left(\mathcal{M}, \mathcal{M}^{\prime}\right)$ as above. It defines a correspondence over $\operatorname{Bun}_{n} \times \operatorname{Bun}_{n}$ :

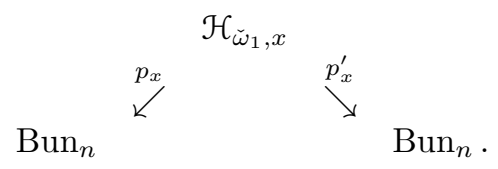

By definition,

$$
\mathbb{H}_{\check{\omega}_{1}, x}(\mathcal{F})=p_{x *} p_{x}^{\prime *}(\mathcal{F}) .
$$

3.4. Hecke functors: general definition. For irreducible representations $\rho_{\mu}$ of ${ }^{L} G$ with general dominant integral highest weights $\mu$, there is an analogous correspondence in which the role of the projective space $\mathbb{P}^{n-1}$ is played by the Schubert variety in the affine Grassmannian of $G$ corresponding to $\mu$.

We explain this in the split case (so that $G=\mathbb{G}$ and ${ }^{L} G=\breve{G}$ ). First, observe that if we have two $G$-bundles $E, E^{\prime}$ on the (formal) disc Spec $k[[t]]$ which are identified over the punctured disc $\operatorname{Spec} k((t))$, we obtain a point in the double quotient

$$
G[[t]] \backslash G((t)) / G[[t]]
$$

or, equivalently, a $G[[t]]$-orbit in the affine Grassmannian

$$
\mathrm{Gr}=G((t)) / G[[t]],
$$

which is an ind-scheme over $k$ [BD, $\mathrm{MV}$. These orbits are called Schubert cells, and they are labeled by elements $\mu$ the set $\mathbf{X}_{+}$of dominant weights of the maximal torus in the dual group ${ }^{L} G$. We denote the orbit corresponding to $\mu$ by $\mathrm{Gr}_{\mu}$. We will write $\operatorname{inv}\left(E, E^{\prime}\right)=\mu$ if the pair $\left(E, E^{\prime}\right)$ belongs to $\operatorname{Gr}_{\mu}$. Note that $\operatorname{Gr}_{\mu^{\prime}}$ is contained in the closure $\overline{\mathrm{Gr}}_{\mu}$ of $\mathrm{Gr}_{\mu}$ if and only if $\mu \geq \mu^{\prime}$.

Following Beilinson and Drinfeld [BD], we introduce the Hecke stack $\mathcal{H}=\mathcal{H}(X, G)$ that classifies quadruples

$$
\left(x, E, E^{\prime}, \phi\right),
$$


where $x \in X, E, E^{\prime} \in \operatorname{Bun}_{G}$ and $\phi$ is an isomorphism

$$
\left.\left.E\right|_{X-\{x\}} \simeq E^{\prime}\right|_{X-\{x\}} .
$$

We have two natural morphisms $p, p^{\prime}: \mathcal{H} \rightarrow \operatorname{Bun}_{G}$ sending such a quadruple to $E$ or $E^{\prime}$ and the morphism $s: \mathcal{H} \rightarrow X$. Since Bun $_{G}$ is an algebraic stack, so is $\mathcal{H}(X, G)$. However, if we fix $E^{\prime}$, then we obtain an ind-scheme over $X$, which is called the Beilinson-Drinfeld Grassmannian (see [BD, $\mathrm{MV}]$ ).

Let $\mathcal{H}^{\prime}(X, G)$ be the stack classifying the quadruples

$$
\left(x, E, E^{\prime}, \phi\right),
$$

where $x \in X, E \in \operatorname{Bun}_{G}, E^{\prime}$ is a $G$-bundle on the disc $D_{x}$ around the point $x$, and $\phi$ is an isomorphism

$$
\left.\left.E\right|_{D_{x}^{\times}} \simeq E^{\prime}\right|_{D_{x}^{\times}}
$$

where $D_{x}^{\times}$is the punctured disc around $x$. We have a natural morphism

$$
\mathcal{H}(X, G) \rightarrow \mathcal{H}^{\prime}(X, G)
$$

(restricting $E^{\prime}$ to $D_{x}$ and $\phi$ to $D_{x}^{\times}$), which is in fact an isomorphism, according to a strong version of a theorem of Beauville-Laszlo [BL given in [BD, Section 2.3.7]. Therefore we obtain that a morphism

$$
s \times p: \mathcal{H}(X, G) \rightarrow X \times \operatorname{Bun}_{G}
$$

sending the above quadruple to $(x, E)$ is a locally trivial fibration with fibers isomorphic to the affine Grassmannian $\mathrm{Gr}=G((t)) / G[[t]]$.

For every dominant integral weight $\mu \in \mathbf{X}_{+}$, we define the closed substack $\mathcal{H}_{\mu}$ of $\mathcal{H}(X, G)$ by imposing the inequality

$$
\operatorname{inv}_{x}\left(E, E^{\prime}\right) \leq \mu .
$$

It is a scheme over $X \times \operatorname{Bun}_{G}$ with fibers isomorphic to $\overline{\mathrm{Gr}}_{\mu}$.

Recall the geometric Satake correspondence [MV], which is an equivalence of tensor categories between the category of finite-dimensional representations of ${ }^{L} G$ and the category of $G[[t]]$-equivariant perverse sheaves on $\mathrm{Gr}$ (see [F1, Sects. 5.45.6], for an exposition). It sends the irreducible finite-dimensional representation $\rho=\rho_{\mu}$ of ${ }^{L} G$ to the irreducible perverse sheaf $\operatorname{IC}\left(\overline{\operatorname{Gr}}_{\mu}\right)$ supported on $\overline{\mathrm{Gr}}_{\mu}$.

Let $\mathcal{K}_{\rho}$ by the perverse sheaf on $\mathcal{H}(X, G)$, supported on $\mathcal{H}_{\mu}$, which is constant along $X \times \operatorname{Bun}_{G}$ with the fibers isomorphic to $\mathrm{IC}\left(\overline{\mathrm{Gr}}_{\mu}\right)$.

We now define the Hecke functor $\mathbb{H}_{\rho}=\mathbb{H}_{\mu}$ as the integral transform corresponding to the kernel $\mathcal{K}_{\rho}$ (see $[\mathrm{BD}]$ ):

$$
\mathbb{H}_{\rho}(\mathcal{F})=(s \times p)_{*}\left(p^{\prime *}(\mathcal{F}) \otimes \mathcal{K}_{\rho}\right) .
$$

For $x \in|X|$, let $\mathcal{H}_{x}$ be the fiber of $\mathcal{H}$ over $x$, and let $p_{x}, p_{x}^{\prime}: \mathcal{H}_{x} \rightarrow \operatorname{Bun}_{G}$ be the corresponding morphisms. Denote by $\mathcal{K}_{\rho, x}$ the restriction of $\mathcal{K}_{\rho}$ to $\mathcal{H}_{x}$. Define the functor $\mathbb{H}_{\rho, x}$ by the formula

$$
\mathbb{H}_{\rho, x}(\mathcal{F})=p_{x *}\left(p_{x}^{*}(\mathcal{F}) \otimes \mathcal{K}_{\rho, x}\right) .
$$

If $X$ is defined over $\mathbb{F}_{q}$, then one can show that the function corresponding to the sheaf $\mathcal{K}_{\rho, x}$ via the Grothendieck dictionary is the kernel $K_{\rho, x}$ of the Hecke operator corresponding to $\rho$ and $x$ (see [F1, Section 5.4]). Therefore the functor $\mathbb{H}_{\rho, x}$ is a geometric analogue of the Hecke operator $\mathbf{H}_{\rho, x}$. 
3.5. Hecke eigensheaves. Let $\mathcal{E}$ be a flat ${ }^{L} G$-bundle on $X$. Then

$$
\rho_{\mathcal{E}}=\mathcal{E} \underset{L_{G}}{\times} \rho
$$

is a flat vector bundle on $X$, hence a $\mathcal{D}$-module on $X$. The following definition is due to $\mathrm{BD}$.

Definition 1. A sheaf $\mathcal{F}$ on $\operatorname{Bun}_{G}$ is called a Hecke eigensheaf with the eigenvalue $\mathcal{E}$ if for any representation $\rho$ of ${ }^{L} G$ we have an isomorphism

$$
\mathbb{H}_{\rho}(\mathcal{F}) \simeq \rho_{\mathcal{E}} \otimes \mathcal{F},
$$

and these isomorphisms are compatible for different $\rho$ with respect to the structures of tensor categories on both sides.

By base change, it follows from the above identity that for every $x \in X$, we have an isomorphism

$$
\mathbb{H}_{\rho, x}(\mathcal{F}) \simeq \rho \otimes \mathcal{F} .
$$

If our curve $X$ is defined over $\mathbb{F}_{q}$, we can pass from a Hecke eigensheaf $\mathcal{F}$ on $\operatorname{Bun}_{G}$ to a function $f$ on $G(F) \backslash G\left(\mathbb{A}_{F}\right) / G\left(\mathcal{O}_{F}\right)$. Then this function will be an eigenfunction of the Hecke operators $\mathbf{H}_{\rho, x}$ :

$$
\mathbf{H}_{\rho, x}(f)=h_{\rho, x} f .
$$

According to the Satake isomorphism (see, e.g., [F1, Section 5.4]), the map

$$
[\rho] \mapsto \mathbf{H}_{\rho, x},
$$

where $\rho$ runs over all finite-dimensional representations of ${ }^{L} G$, defines an isomorphism between the representation $\operatorname{ring} \operatorname{Rep}^{L} G$ of ${ }^{L} G$ and the spherical Hecke algebra generated by $\mathbf{H}_{\rho, x}$. Hence the collection of eigenvalues $\left\{h_{\rho, x}\right\}$ defines a point in the spectrum of $\operatorname{Rep}{ }^{L} G$, that is, a semi-simple conjugacy class $\nu_{x}$ in ${ }^{L} G$.

If the (unramified) automorphic representation $\pi$ generated by the spherical function $f$ corresponds to a homomorphism $\sigma: W(F) \rightarrow{ }^{L} G$ under the Langlands correspondence (2.6), then $\nu_{x}=\sigma\left(\mathrm{Fr}_{x}\right)$, where $\mathrm{Fr}_{x}$ is the Frobenius conjugacy class associated to $x$ (see [F1, Section 2.2]). In other words, in this case

$$
h_{\rho, x}=\operatorname{Tr}\left(\sigma\left(\mathrm{Fr}_{x}\right), \rho\right)
$$

(up to a power of $q$ ). A general automorphic representation $\pi$ would be ramified at finitely many points $x \in|X|$. Then this condition would only be satisfied away from those points. This is the precise meaning of the matching between the Hecke and Frobenius eigenvalues that we mentioned above.

3.6. Geometric Langlands correspondence. Now we can state the geometric Langlands correspondence as the following diagram.

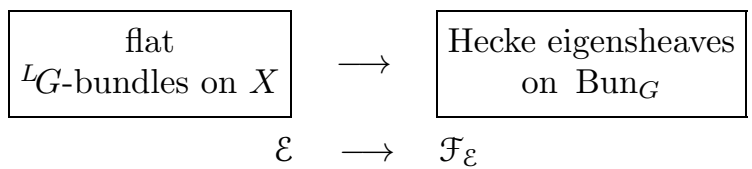

This correspondence has been constructed in many cases. For $G=G L_{n}$ the Hecke eigensheaves corresponding to irreducible $\mathcal{E}$ have been constructed in FGV2, G], building on the work of P. Deligne for $n=1$ (explained in [Lau3] and [F1]), V. Drinfeld [D1] for $n=2$, and G. Laumon [Lau3] (this construction works for curves defined both over $\mathbb{F}_{q}$ or $\mathbb{C}$ ). 
For any split simple algebraic group $G$ and $X$ defined over $\mathbb{C}$, the Hecke eigensheaves have been constructed in a different way by A. Beilinson and V. Drinfeld $\mathrm{BD}$, in the case that $\mathcal{E}$ has an additional structure of an oper (this means that $\mathcal{E}$ belongs to a certain half-dimensional locus in $\left.\operatorname{Loc}_{L_{G}}\right)$. It is interesting that this construction is closely related to the 2D Conformal Field Theory and representation theory of affine Kac-Moody algebras of critical level. For more on this, see [F1, Part III].

3.7. Categorical version. Looking at the correspondence (3.9), we notice that there is an essential asymmetry between the two sides. On the left we have flat ${ }^{L} G$-bundles, which are points of a moduli stack $\operatorname{Loc}_{L_{G}}$ of flat ${ }^{L} G$-bundles (or local systems) on $X$. But on the right we have Hecke eigensheaves, which are objects of a category; namely, the category of $\mathcal{D}$-modules on $\operatorname{Bun}_{G}$. Beilinson and Drinfeld have suggested how to formulate it in a more symmetrical way.

The idea is to replace a point $\mathcal{E} \in \operatorname{Loc}_{L_{G}}$ by an object of another category; namely, the skyscraper sheaf $\mathcal{O}_{\mathcal{E}}$ at $\mathcal{E}$ viewed as an object of the category of coherent $\mathcal{O}$-modules on $\operatorname{Loc}_{L}$. A much stronger, categorical, version of the geometric Langlands correspondence is then a conjectural equivalence of derived categories 2

$$
\begin{array}{|c|}
\hline \text { derived category of } \\
\mathcal{O} \text {-modules on } \operatorname{Loc}_{L} G
\end{array} \longleftrightarrow \begin{array}{c|}
\text { derived category of } \\
\mathcal{D} \text {-modules on } \operatorname{Bun}_{G}
\end{array}
$$

This equivalence should send the skyscraper sheaf $\mathcal{O}_{\mathcal{E}}$ on $\operatorname{Loc}_{L_{G}}$ supported at $\mathcal{E}$ to the Hecke eigensheaf $\mathcal{F}_{E}$. If this were true, it would mean that Hecke eigensheaves provide a good "basis" in the category of $\mathcal{D}$-modules on $\mathrm{Bun}_{G}$, so we would obtain a kind of spectral decomposition of the derived category of $\mathcal{D}$-modules on $\operatorname{Bun}_{G}$, like in the Fourier transform. (Recall that under the Fourier transform on the real line, the delta-functions $\delta_{x}$, analogues of $\mathcal{O}_{\mathcal{E}}$, go to the exponential functions $e^{i t x}$, analogues of $\mathcal{F}_{\mathcal{E}}$.)

This equivalence has been proved by G. Laumon [Lau2 and M. Rothstein $\mathrm{R}$ ] in the abelian case, when $G=G L_{1}$ (or a more general torus). They showed that in this case this is nothing but a version of the Fourier-Mukai transform. Thus, the categorical Langlands correspondence may be viewed as a kind of non-abelian Fourier-Mukai transform (see [F1, Section 4.4]).

In the non-abelian case, this has not yet been made into a precise conjecture in the literature 3 Nevertheless, the diagram (3.10) gives us a valuable guiding principle to the geometric Langlands correspondence. In particular, it gives us a natural explanation as to why the skyscraper sheaves on $\operatorname{Loc}_{L_{G}}$ should correspond to Hecke eigensheaves.

The point is that on the category of $\mathcal{O}$-modules on $\operatorname{Loc}_{L_{G}}$ we also have a collection of functors $\mathbb{W}_{V}$, parametrized by the same data as the Hecke functors $\mathbb{H}_{V}$. We will call them the Wilson functors (because of the close connection between them and the Wilson line operators in 4D gauge theory). These functors act from the

\footnotetext{
${ }^{2}$ It is expected (see $\left[\mathrm{FW}\right.$, Section 10]) that there is in fact a $\mathbb{Z}_{2}$-gerbe of such equivalences. This gerbe is trivial, but not canonically trivialized. One gets a particular trivialization of this gerbe, and hence a particular equivalence, for each choice of the square root of the canonical line bundle $K_{X}$ on $X$.

${ }^{3}$ After I presented these Colloquium Lectures, the paper AG appeared in which a precise formulation of this conjecture was proposed.
} 
category of $\mathcal{O}$-modules on $\operatorname{Loc}_{L_{G}}$ to the category of sheaves on $X \times \operatorname{Loc}_{L_{G}}$, which are $\mathcal{D}$-modules along $X$ and $\mathcal{O}$-modules along $\operatorname{Loc}_{L_{G}}$.

To define them, observe that we have a tautological ${ }^{L} G$-bundle $\mathcal{T}$ on $X \times \operatorname{Loc}_{L} G$, whose restriction to $X \times \mathcal{E}$, where $\mathcal{E}=(E, \nabla)$, is $E$. Moreover, $\nabla$ gives us a partial connection on $\mathcal{T}$ along $X$. For a representation $V$ of ${ }^{L} G$, let $\mathcal{T}_{V}$ be the associated vector bundle on $X \times \operatorname{Loc}_{L}$, with a connection along $X$.

Let $p_{2}: X \times \operatorname{Loc}_{L_{G}} \rightarrow \operatorname{Loc}_{L_{G}}$ be the projection onto the second factor. By definition,

$$
\mathbb{W}_{V}(\mathcal{F})=\mathcal{T}_{V} \otimes p_{2}^{*}(\mathcal{F})
$$

(note that by construction $\mathcal{T}_{V}$ carries a connection along $X$ and so the right-hand side really is a $\mathcal{D}$-module along $X$ ).

Now, the conjectural equivalence (3.10) should be compatible with the Wilson/Hecke functors in the sense that

$$
C\left(\mathbb{W}_{V}\right) \simeq \mathbb{H}_{V}, \quad V \in \operatorname{Rep}^{L} G,
$$

where $C$ denotes this equivalence (from left to right).

In particular, observe that the skyscraper sheaf $\mathcal{O}_{\mathcal{E}}$ at $\mathcal{E} \in \operatorname{Loc}_{L_{G}}$ is obviously an eigensheaf of the Wilson functors:

$$
\mathbb{W}_{V}\left(\mathcal{O}_{\mathcal{\varepsilon}}\right)=V_{\mathcal{E}} \otimes \mathcal{O}_{\varepsilon}
$$

Indeed, tensoring a skyscraper sheaf with a vector bundle is the same as tensoring it with the fiber of this vector bundle at the point of support of this skyscraper sheaf. Therefore (3.12) implies that $\mathcal{F}_{\mathcal{E}}=C\left(\mathcal{O}_{\mathcal{E}}\right)$ must satisfy the Hecke property (3.5). In other words, $\mathcal{F}_{\mathcal{E}}$ should be a Hecke eigensheaf on $\operatorname{Bun}_{G}$ with eigenvalue $\mathcal{E}$. Thus, we obtain a natural explanation of the Hecke property of $\mathcal{F}_{\mathcal{E}}$ : it follows from the compatibility of the categorical Langlands correspondence (3.10) with the Wilson/Hecke functors.

Thus, the conjectural equivalence (3.10) gives us a natural and convenient framework for the geometric Langlands correspondence.

The equivalence (3.10) also arises in the study of S-duality of the maximally 4D supersymmetric gauge theories with the gauge groups being the compact forms of $G$ and ${ }^{L} G$. As shown by Kapustin and Witten [KW] (see [F2 for an exposition), the S-duality of boundary conditions in these theories yields an equivalence closely related to (3.10), in which the category of $\mathcal{D}$-modules on $\operatorname{Bun}_{G}$ is replaced by the category of $A$-branes on the cotangent bundle of $\mathrm{Bun}_{G}$.

\section{LANGLANDS FUNCTORIALITY AND TRACE FORMULA}

The Langlands correspondence (2.6) is subtle, because it involves objects from two different worlds: automorphic representations and Galois representations. However, there is a closely related correspondence that may be formulated entirely in the world of automorphic representations.

4.1. The Langlands Functoriality Principle. Let $G$ and $H$ be two reductive algebraic groups over the function field $F$ of smooth projective curve $X$ over a finite field, and assume that $G$ is quasi-split (that is, contains a Borel subgroup defined over $F$ ). Let ${ }^{L} G$ and ${ }^{L} H$ be their Langlands dual groups and

$$
a:{ }^{L} H \rightarrow{ }^{L} G
$$


a homomorphism between them that induces the identity on the Galois parts. Such homomorphisms are called admissible.

Given a homomorphism $\sigma: W(F) \rightarrow{ }^{L} H$, we obtain a homomorphism $a \circ \sigma$ : $W(F) \rightarrow{ }^{L} G$, and hence a natural map of sets of equivalence classes

$$
\begin{gathered}
\begin{array}{c}
\text { homomorphisms } \\
W(F) \rightarrow{ }^{L} H
\end{array} \\
\downarrow \\
\begin{array}{c}
\text { homomorphisms } \\
W(F) \rightarrow{ }^{L} G
\end{array}
\end{gathered}
$$

Taking into account the Langlands correspondence (2.6) for the group $G$ and the one for $H$, we conclude that to each $L$-packet of tempered automorphic representations of $H\left(\mathbb{A}_{F}\right)$ should correspond an $L$-packet of automorphic representations of $G\left(\mathbb{A}_{F}\right)$. In fact, there should be a map

$L$-packets of automorphic representations of $H\left(\mathbb{A}_{F}\right)$

$$
\begin{aligned}
& L \text {-packets of automorphic } \\
& \text { representations of } G\left(\mathbb{A}_{F}\right)
\end{aligned}
$$

It is called the Langlands Functoriality or transfer of automorphic representations.

The existence of such a map is non-trivial and surprising, because even though we have a homomorphism of dual groups $a:{ }^{L} H \rightarrow{ }^{L} G$, there is a priori no connection between the groups $G$ and $H$.

These transfers should have the following associativity property: if $M$ is another reductive group and we have a chain of homomorphisms:

$$
{ }^{L} M \rightarrow{ }^{L} H \rightarrow{ }^{L} G,
$$

then the two transfers of automorphic representations from $M\left(\mathbb{A}_{F}\right)$ to $G\left(\mathbb{A}_{F}\right)$, one obtained as the composition of the transfers from $M\left(\mathbb{A}_{F}\right)$ to $H\left(\mathbb{A}_{F}\right)$ and from $H\left(\mathbb{A}_{F}\right)$ to $G\left(\mathbb{A}_{F}\right)$, and the other obtained directly from the composition ${ }^{L} M \rightarrow{ }^{L} G$, should coincide.

In addition, we require that under the transfer the Hecke eigenvalues of automorphic representations should match, in the following sense. Recall from Section 3.5 that the eigenvalues of the Hecke operators assign to an automorphic representation of $G\left(\mathbb{A}_{F}\right)$ a collection of conjugacy classes $\left(\nu_{x}\right)$, for all but finitely many $x \in|X|$, in the Langlands dual group ${ }^{L} G$. It is known that for all automorphic representations $\pi_{j}$ of $G\left(\mathbb{A}_{F}\right)$ that belong to a given $L$-packet $\left\{\pi_{j}\right\}$ these conjugacy classes are the same.

Now, let $\left\{\pi_{i}^{H}\right\}$ be an $L$-packet of automorphic representations of $H\left(\mathbb{A}_{F}\right)$ and $\left(\nu_{x}^{H}\right)$ the collection of ${ }^{L} H$-conjugacy classes assigned to it. Let $\left\{\pi_{j}^{G}\right\}$ be the $L$ packet of automorphic representations which is the transfer of $\left\{\pi_{i}^{H}\right\}$ under (4.1), 
and $\left(\nu_{x}^{G}\right)$ the collection of ${ }^{L} G$-conjugacy classes assigned to it. Then they should be defined for the same $x \in|X|$ as the $\nu_{x}^{H}$, and for each of these $x \in|X|$ we must have

$$
\nu_{x}^{G}=a\left(\nu_{x}^{H}\right) .
$$

Functoriality has been established in some cases, but is unknown in general (see Art1 for a survey).

4.2. Geometric functoriality. Given a homomorphism ${ }^{L} H \rightarrow{ }^{L} G$, we obtain a natural morphism of algebraic stacks $\operatorname{Loc}_{L_{H}} \rightarrow \operatorname{Loc}^{L} G$ and hence a natural functor (direct image) from the category of $\mathcal{O}$-modules on $\operatorname{Loc}_{L_{H}}$ to that on $\operatorname{Loc}_{L_{G}}$. Hence, in view of the categorical Langlands correspondence (3.10), we should also have a functor from the derived category of $\mathcal{D}$-modules on $\mathrm{Bun}_{H}$ to that on $\mathrm{Bun}_{G}$, making the following diagram commutative.

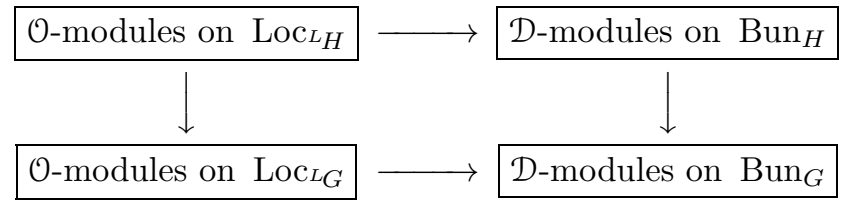

The right vertical arrow is the geometric functoriality functor. Examples have been constructed in Ly1, Ly2 (see also LafL) using a geometric version of the theta-correspondence. In [FW] the geometric functoriality for endoscopic groups was analyzed using the Mirror Symmetry approach to the geometric Langlands correspondence (in this setting the category of $\mathcal{D}$-modules on $\operatorname{Bun}_{G}$ is replaced by the category of $A$-branes on the cotangent bundle of $\mathrm{Bun}_{G}$ ).

4.3. Non-tempered representations. In [L2, L3] Langlands proposed a strategy for proving the Functoriality Conjecture, which is based on the use of the trace formula. This was further developed in [FLN] and [L4. In order to explain this, we need to discuss first the non-tempered representations and a modified version of the functoriality transfer. Then, in the next subsection, we will introduce the trace formula, and in Sections 4.5 4.10 we will explain the strategy of [FLN] to use the trace formula to prove the Functoriality Conjecture.

Recall that according to the Langlands correspondence (formula (2.6)), the $L$ packets of tempered automorphic representations are supposed to be parametrized by the equivalence classes of homomorphisms $W(F) \rightarrow{ }^{L} G$. According to the conjectures of Arthur, for general automorphic representations of $G\left(\mathbb{A}_{F}\right)$ the homomorphisms $W(F) \rightarrow{ }^{L} G$ should be replaced by the Arthur parameters: equivalence classes of homomorphisms

$$
\sigma: \mathrm{SL}_{2} \times W(F) \rightarrow{ }^{L} G
$$

that induce the canonical map $W(F) \rightarrow \operatorname{Gal}(\bar{F} / F) \rightarrow \Gamma$.

If an irreducible automorphic representation $\pi$ is tempered, then $\left.\sigma\right|_{\mathrm{SL}_{2}}$ is trivial. Those $\sigma$ for which $\left.\sigma\right|_{\mathrm{SL}_{2}}$ is non-trivial, correspond to non-tempered automorphic representations.

If an automorphic representation

$$
\pi=\bigotimes_{x \in|X|}^{\prime} \pi_{x}
$$


of the adelic group $G\left(\mathbb{A}_{F}\right)$ has the Arthur parameter $\sigma$, then for all closed points $x \in|X|$ where $\pi_{x}$ is unramified the restriction $\left.\sigma\right|_{W(F)}$ is also unramified and the conjugacy class $\nu_{x}$ in ${ }^{L} G$ corresponding to $\pi_{x}$ is the conjugacy class of

$$
\sigma\left(\left(\begin{array}{cc}
q^{1 / 2} & 0 \\
0 & q^{-1 / 2}
\end{array}\right) \times \operatorname{Fr}_{x}\right) \in{ }^{L} G
$$

Let $\sigma$ be an Arthur parameter. We attach to it two subgroups of ${ }^{L} G:{ }^{\lambda} G={ }^{\lambda} G_{\sigma}$ which is the centralizer of the image of $\mathrm{SL}_{2}$ in ${ }^{L} G$ under $\sigma$, and ${ }^{\lambda} H={ }^{\lambda} H_{\sigma}$ is the Zariski closure of the image of $W(F)$ in ${ }^{\lambda} G_{\sigma}$ under $\sigma$.

Technical point. The group ${ }^{\lambda} H$ may not be a Langlands dual group, but the image of an admissible homomorphism ${ }^{L} H \rightarrow{ }^{L} G$ (so that we have a surjection $\left.{ }^{L} H \rightarrow{ }^{\lambda} H\right)$. As explained on L2, Sections 1.4 and 1.7], we may enlarge ${ }^{\lambda} H$ by a central torus to get a Langlands dual group ${ }^{L} H$. In what follows we will ignore this issue.

The idea of Langlands [L2] (see also [FLN, L5]) is to assign (bypassing Arthur parameters) to each irreducible automorphic representations $\pi$ of $G\left(\mathbb{A}_{F}\right)$ (more precisely, an $L$-packet) directly the data of

$$
\phi: \mathrm{SL}_{2} \times{ }^{L} H \rightarrow{ }^{\lambda} H \hookrightarrow{ }^{L} G
$$

and a tempered irreducible automorphic representation $\pi_{H}$ (or an $L$-packet) of the group $H\left(\mathbb{A}_{F}\right)$ whose dual group is ${ }^{L} H$, which Langlands calls hadronic. Note that the same $\pi$ might correspond to several inequivalent homomorphisms $\phi$, but this is expected to be related to the multiplicity of $\pi$ in the space of automorphic functions.

From this point of view, $\pi$ should be thought of as a transfer of $\pi_{H}$ with respect to $\phi$, so we obtain a more convenient notion of transfer for non-tempered representations which explicitly involves Arthur's group $\mathrm{SL}_{2}$ (note that it is different from the transfer discussed in Section 4.1). According to formula (4.3), under this transfer the Hecke eigenvalues $\left(\nu_{x}^{H}\right)$ of $\pi_{H}$ and $\left(\nu_{x}\right)$ of $\pi$ should be matched by the formula

$$
\nu_{x}=\phi\left(\left(\begin{array}{cc}
q^{1 / 2} & 0 \\
0 & q^{-1 / 2}
\end{array}\right) \times \nu_{x}^{H}\right) .
$$

Thus, we obtain a conjectural description of the equivalence classes of automorphic representations of all reductive groups $G$ in terms of the pairs $\left(\phi, \pi_{H}\right)$, where $\pi_{H}$ is hadronic, and $\phi$ is a homomorphism (4.4). In [L2, FLN] (see also L4, L5) a strategy for proving it using the trace formula was suggested. We discuss it next.

4.4. Trace formula. Let $f$ be a smooth compactly supported function on $G\left(\mathbb{A}_{F}\right)$. We choose a Haar measure on $G\left(\mathbb{A}_{F}\right)$ normalized so that the volume of the fixed maximal compact subgroup

$$
G\left(\mathcal{O}_{F}\right)=\prod_{x \in|X|} G\left(\mathcal{O}_{x}\right)
$$

is equal to 1 . Denote by $\mathbf{K}_{f}$ the operator on the space of automorphic functions on $G(F) \backslash G\left(\mathbb{A}_{F}\right)$ acting by the formula

$$
\left(\mathbf{K}_{f} \cdot \psi\right)(x)=\int_{G(\mathbb{A})} \psi(x y) f(y) d y .
$$

Thus, we "average" the right action of $y \in G\left(\mathbb{A}_{F}\right)$ with the "weight" $f(y)$. 
We rewrite $\mathbf{K}_{f}$ as an integral operator corresponding to the kernel

$$
K_{f}(x, y)=\sum_{a \in G(F)} f\left(x^{-1} a y\right)
$$

acting as follows:

$$
\left(\mathbf{K}_{f} \cdot \psi\right)(x)=\int_{G(F) \backslash G(\mathbb{A})} K_{f}(x, y) \psi(y) d y .
$$

The Arthur-Selberg trace formula for $\mathbf{K}$ reads, formally,

$$
\operatorname{Tr} \mathbf{K}_{f}=\int_{G(F) \backslash G(\mathbb{A})} K_{f}(x, x) d x .
$$

This is correct if $G(F) \backslash G\left(\mathbb{A}_{F}\right)$ is compact, in which case there is no continuous spectrum; otherwise, some important modifications need to be made. We will not discuss this here, referring the reader to Art2 and references therein.

The left-hand side of this formula, called the spectral side, may be rewritten as follows (we ignore the continuous spectrum):

$$
\sum_{\pi} m_{\pi} \operatorname{Tr}\left(\mathbf{K}_{f}, \pi\right)
$$

where the sum is over the irreducible automorphic representations $\pi$ of $G\left(\mathbb{A}_{F}\right)$ and $m_{\pi}$ is the multiplicity of $\pi$ in the space of automorphic functions.

The right-hand side of (4.7) may be rewritten as (see Art2, Section 1)

$$
\sum_{\gamma \in G(F) / \text { conj. }} a_{\gamma} O_{\gamma}(f),
$$

where $\gamma$ runs over the set of conjugacy classes in $G(F), O_{\gamma}(f)$ is the global orbital integral defined by the formula

$$
O_{\gamma}(f)=\int_{G_{\gamma}\left(\mathbb{A}_{F}\right) \backslash G\left(\mathbb{A}_{F}\right)} f\left(g^{-1} \gamma g\right) d g,
$$

and

$$
a_{\gamma}=\operatorname{vol}\left(G_{\gamma}(F) \backslash G_{\gamma}\left(\mathbb{A}_{F}\right)\right) .
$$

Here $G_{\gamma}(F)$ (resp., $G_{\gamma}\left(\mathbb{A}_{F}\right)$ ) denotes the stabilizer of $\gamma$ in $G(F)$ (resp., $G\left(\mathbb{A}_{F}\right)$ ).

The sum (4.9) is usually called the geometric side of the trace formula, but we will call it the orbital side, because by its geometrization we will understand its representation as the trace of the Frobenius on a vector space.

Thus, the trace formula (4.7) takes the form

$$
\sum_{\pi} m_{\pi} \operatorname{Tr}\left(\mathbf{K}_{f}, \pi\right)=\sum_{\gamma \in G(F) / \text { conj. }} a_{\gamma} O_{\gamma}(f) .
$$

We want to use this formula to establish the functoriality transfer (4.1). The idea is to find enough relations between $\operatorname{Tr} \mathbf{K}_{f}$ and $\operatorname{Tr} \mathbf{K}_{f^{H}}$ for a sufficiently large class of functions $f$ on $G\left(\mathbb{A}_{F}\right)$ and a suitable map

$$
f \mapsto f^{H},
$$


where $f^{H}$ is a function on $H\left(\mathbb{A}_{F}\right)$. Though the formula 4.10) for a single function $f$ does not necessarily pin down a particular irreducible representation $\pi$ - the lefthand side of (4.10) is a sum over those - if we have at our disposal formulas for the trace $\operatorname{Tr} \mathbf{K}_{f}$ for a sufficiently large class of functions $f$, then we can separate different irreducible representations. So if we can prove enough relations between $\operatorname{Tr} \mathbf{K}_{f}$ and $\operatorname{Tr} \mathbf{K}_{f^{H}}$, then we can often derive the existence of an irreducible automorphic representation $\pi$ of $G\left(\mathbb{A}_{F}\right)$ whose Hecke eigenvalues match those of an irreducible automorphic representation $\pi_{H}$ of $H\left(\mathbb{A}_{F}\right)$ (or, in general, $L$-packets of those).

In order to find these relations between the traces, we look for relations between the global orbital integrals $O_{\gamma}(f)$ and $O_{\gamma^{H}}\left(f^{H}\right)$ appearing on the right-hand side of (4.10) for $G\left(\mathbb{A}_{F}\right)$ and $H\left(\mathbb{A}_{F}\right)$, respectively. This should eventually be reduced to proving relations between local orbital integrals of the local factors $f_{x}$ and $f_{x}^{H}$ of $f=\bigotimes_{x} f_{x}$ and $f^{H}=\bigotimes_{x} f_{x}^{H}$, respectively. Thus, everything should boil down to a problem in local harmonic analysis. This is the basic strategy used to prove the Functoriality Conjecture.

This strategy has been successfully employed in a number of cases. Perhaps, the most famous (and historically one of the first) examples is the Jacquet-Langlands theory JL. Here $G=G L_{2}$ and $H$ is the multiplicative group of a quaternion algebra over $F$, which is an inner form of $G$. Thus, ${ }^{L} G={ }^{L} H=G L_{2}$, and we take the identity as the homomorphism $a$ between them. Jacquet and Langlands give a very explicit construction of the transfer of functions and conjugacy classes under which the orbital integrals for the two groups are equal. This, together with the strong multiplicity one theorem for $G L_{2}$ (which says that the collection of conjugacy classes $\left(\nu_{x}\right)$ at almost all points $x \in|X|$ uniquely determine an irreducible automorphic representation of $G L_{2}\left(\mathbb{A}_{F}\right)$ ), allows them to prove the existence of the transfer $\pi_{H} \mapsto \pi$ satisfying the above properties.

Technical point. Labesse and Langlands have shown in [LL that the same strategy fails already in the case of $G=\mathrm{SL}_{2}$. It turns out that for general groups one needs first to "stabilize" the trace formula. Roughly speaking, this allows us to write the orbital side in terms of the products, over $x \in|X|$, of local stable orbital integrals. Here "stable" refers to "stable conjugacy class" in $G\left(F_{x}\right)$, the union of the conjugacy classes in $G\left(F_{x}\right)$ that are conjugate to each other over the algebraic closure of $F_{x}$ (if $G=G L_{n}$, each stable conjugacy class consists of one conjugacy class, but for other groups it may consist of several conjugacy classes). Stable orbital integrals for different groups may then be compared. Fortunately, the stabilization of trace formulas has now been achieved thanks to Ngô's recent proof of the fundamental lemma [N2] and other important results.

One of the benefits of the stabilization of the trace formula is that it leads to the proof of the Functoriality Conjecture for the so-called endoscopic groups (at least, those of classical types). These are the the groups $H$ whose Langlands dual groups ${ }^{L} H$ are, roughly speaking, the centralizers of semi-simple elements in ${ }^{L} G$ (see, e.g., N2] for a precise definition).

4.5. Strategy. In [FLN], following [L2, L3] (see also [L4, L5, FN]), the following strategy for proving functoriality has been proposed.

Suppose we had at our disposal an explicitly defined family of integral operators $\left\{\mathbf{K}_{f_{i}}\right\}$ on the space of automorphic functions on $G(F) \backslash G\left(\mathbb{A}_{F}\right)$ which annihilate all automorphic representations of $G\left(\mathbb{A}_{F}\right)$ that do not come by transfer from automorphic representations of $H\left(\mathbb{A}_{F}\right)$. Then we will be able to isolate in $L_{2}\left(G(F) \backslash G\left(\mathbb{A}_{F}\right)\right)$ 
those representations that come by functoriality from automorphic representations of $H\left(\mathbb{A}_{F}\right)$. Hence we may compare the traces of these operators with the traces of some operators $\left\{\mathbf{K}_{f_{i}^{H}}\right\}$ acting on $L_{2}\left(H(F) \backslash H\left(\mathbb{A}_{F}\right)\right)$ using the corresponding orbital integrals.

While it is not known how to do this literally for any given pair of groups $G$ and $H$, operators with a similar property have indeed been constructed in [L2, FLN].

In what follows we will describe a class of such operators, denoted by $\mathbf{K}_{d, \rho}$, defined in FLN, restricting ourselves for simplicity to unramified automorphic representations (for the general case, see [FLN]). In this case $f$ is a finite linear combination of functions of the form $\bigotimes_{x} f_{x}$, where each $f_{x}$ is in the spherical Hecke algebra of $G\left(F_{x}\right)$ (with respect to $G\left(\mathcal{O}_{x}\right)$ ), and $f_{x}=1$ for all but finitely many $x \in|X|$.

The operators $\mathbf{K}_{d, \rho}$ depend on a positive integer $d$ and an irreducible representation $\rho$ of ${ }^{L} G$ and are expected to have the following property: for sufficiently large $d$, the representations of $G\left(\mathbb{A}_{F}\right)$ that contribute to the trace of $\mathbf{K}_{d, \rho}$ are those coming by functoriality from the groups $H$ satisfying the following.

Property $(G, \rho)$ : The pull-back $a^{*}(\rho)$ of $\rho$ to ${ }^{L} H$ under the homomorphism $a$ : ${ }^{L} H \rightarrow{ }^{L} G$ has non-zero invariant vectors.

This suggests a path to proving functoriality: we need to express the right-hand side of the trace formula for $\mathbf{K}_{d, \rho}$ as the sum of orbital integrals and compare these orbital integrals for $G$ and the groups $H$ satisfying property $(G, \rho)$.

We will now give a simple example of a group satisfying this property and then define the operators $\mathbf{K}_{d, \rho}$. Then we will compute the eigenvalues of these operators on the Hecke eigenfunctions in Section 4.8. Using this computation, we will derive the crucial Lemma 1 which shows that for large enough $d$ the operator $\mathbf{K}_{d, \rho}$ annihilates the Hecke eigenfunctions that do not come by functoriality from the groups $H$ satisfying property $(G, \rho)$.

4.6. Example. What are the possible groups $H$ with the $(G, \rho)$ property? Consider the case of $G=G L_{2}$, then ${ }^{L} G=G L_{2}$ as well. Let $\rho=\rho_{1}$, the defining two-dimensional representation of $G L_{2}$. We are interested in the reductive subgroups ${ }^{L} H$ that stabilize proper non-zero subspaces of $\rho$. In this case this subspace has to be a line in the two-dimensional vector space. The group ${ }^{L} H$ is then a onedimensional torus. This is not a very interesting example, because functoriality for tori is established via the Eisenstein series.

Let us now consider the three-dimensional representation $\rho_{2}=\operatorname{Sym}^{2}\left(\rho_{1}\right)$. If we choose a basis $\left\{v_{1}, v_{2}\right\}$ of $\rho_{1}$, then $\rho_{2}$ has the basis

$$
\left\{v_{1} \otimes v_{1}, v_{1} \otimes v_{2}+v_{2} \otimes v_{1}, v_{2} \otimes v_{2}\right\} .
$$

Then in addition to the one-dimensional torus stabilizing the vector $v_{1} \otimes v_{1}$, we will have another group with the $\left(G L_{2}, \rho_{2}\right)$ property, $O_{2}=\mathbb{Z}_{2} \ltimes G L_{1}$, stabilizing the line spanned by the second vector. This group consists of the matrices

$$
\left(\begin{array}{cc}
x & 0 \\
0 & x^{-1}
\end{array}\right), \quad\left(\begin{array}{cc}
x & 0 \\
0 & x^{-1}
\end{array}\right)\left(\begin{array}{ll}
0 & 1 \\
1 & 0
\end{array}\right) .
$$

It is the Langlands dual group of the twisted tori described in Section 2.5. Hence the corresponding groups $H$ are the twisted tori in this case. 
4.7. Definition of $\mathbf{K}_{d, \rho}$. Let $X$ be defined over $k=\mathbb{F}_{q}$. Assume for simplicity that $G$ is split over $k$ (a more general case is considered in [FN]). Recall the Hecke operator $\mathbf{H}_{\rho, x}, x \in|X|, \rho \in \operatorname{Rep}^{L} G$, and its kernel $K_{\rho, x}$, which is a function on $\operatorname{Bun}_{G}(k) \times \operatorname{Bun}_{G}(k)$.

We define the kernel $K_{d, \rho}$ for $d \geq 1$ on $\operatorname{Bun}_{G}(k) \times \operatorname{Bun}_{G}(k)$.

For $d=1$ it is simply the sum of $K_{\rho, x}$ over all $x \in X(k)$ :

$$
K_{1, \rho}=\sum_{x \in|X|} K_{\rho, x}
$$

For $d=2$, we want to define the symmetric square of $K_{1, \rho}$. In other words, we sum over the degree two effective divisors $D$-these are the $k$-points in the symmetric square $X^{(2)}=X^{2} / S_{2}$ of our curve $X$. There are three types of such divisors: $D=(x)+(y)$, where $x, y \in X(k), x \neq y$-to which we assign $K_{\rho, x} K_{\rho, y}$; $D=(x)$, where $x \in X\left(\mathbb{F}_{q^{2}}\right)$ - we assign $K_{\rho, x}$; and $D=2(x)$ - then naively we could assign $K_{\rho, x}^{2}=K_{\rho^{\otimes 2}, x}$, but since we want the symmetric product, we assign instead $K_{\rho^{(2)}, x}$, where

$$
\rho^{(2)}=\operatorname{Sym}^{2}(\rho) \text {. }
$$

Similarly, for $d>2$ we set

$$
K_{d, \rho}=\sum_{D \in X^{(d)}(k)} \prod_{i} K_{\rho^{\left(n_{i}\right)}, x_{i}}, \quad D=\sum_{i} n_{i}\left[x_{i}\right]
$$

where

$$
\rho^{(n)}=\operatorname{Sym}^{n}(\rho) \text {. }
$$

Let $\mathbf{K}_{d, \rho}$ be the integral operator on functions on $\operatorname{Bun}_{G}(k)$ corresponding to the kernel $K_{d, \rho}$. Thus,

$$
\mathbf{K}_{d, \rho}=\sum_{D \in X^{(d)}(k)} \prod_{i} \mathbf{H}_{\rho^{\left(n_{i}\right)}, x_{i}}, \quad D=\sum_{i} n_{i}\left[x_{i}\right] .
$$

4.8. Eigenvalues of $\mathbf{K}_{d, \rho}$. Now let $f_{\sigma}$ be a Hecke eigenfunction on $\operatorname{Bun}_{G}(k)$ with respect to an unramified homomorphism $\sigma: W(F) \rightarrow{ }^{L} G$. Recall from formulas (3.7) and (3.8) that

$$
\mathbf{H}_{V, x} \cdot f_{\sigma}=\operatorname{Tr}\left(\sigma\left(\mathrm{Fr}_{x}\right), V\right) f_{\sigma}
$$

for any finite-dimensional representation $V$ of ${ }^{L} G$.

Therefore we find from formula (4.12) that

$$
\mathbf{K}_{d, \rho} \cdot f_{\sigma}=l_{d, \rho} f_{\sigma},
$$

where

$$
l_{d, \rho}=\sum_{D \in X^{(d)}(k)} \prod_{i} \operatorname{Tr}\left(\sigma\left(\operatorname{Fr}_{x_{i}}\right), \rho^{\left(n_{i}\right)}\right), \quad D=\sum_{i} n_{i}\left[x_{i}\right] .
$$

Consider the generating function of these eigenvalues:

$$
L(\sigma, \rho, t)=\sum_{d \geq 0} l_{d, \rho} t^{d}=\prod_{x \in|X|} \operatorname{det}\left(1-t^{\operatorname{deg}(x)} \sigma\left(\mathrm{Fr}_{x}\right), \rho\right)^{-1} .
$$

If we substitute $t=q^{-s}$, we obtain the $L$-function $L\left(\sigma, \rho, q^{-s}\right)$ attached to $\sigma$ and $\rho$. Thus, formula (4.13) implies that the eigenvalues of $\mathbf{K}_{d, \rho}$ are the coefficients of this $L$-function:

$$
\mathbf{K}_{d, \rho} \cdot f_{\sigma}=\left(q^{-d s} \text {-coefficient of } L\left(\sigma, \rho, q^{-s}\right)\right) f_{\sigma} .
$$


Lemma 1. Suppose that the spaces of invariants and coinvariants of the representation $\rho \circ \sigma$ of the Weil group $W(F)$ are equal to 0 . Then the corresponding Hecke eigenfunction $f_{\sigma}$ satisfies

$$
\mathbf{K}_{d, \rho} \cdot f_{\sigma}=0, \quad d>2(g-1) \operatorname{dim} \rho,
$$

where $g$ is the genus of $X$.

The same statement holds if $f_{\sigma}$ is a Hecke eigenfunction corresponding to an Arthur parameter $\sigma: \mathrm{SL}_{2} \times W(F) \rightarrow{ }^{L} G$ and the spaces of invariants and coinvariants of the representation $\left.\rho \circ \sigma\right|_{W(F)}$ of the Weil group $W(F)$ are equal to 0 .

Corollary 2. Let ${ }^{\lambda} H_{\sigma}$ be the Zariski closure of the image of $W(F)$ in ${ }^{\lambda} G_{\sigma}$ under $\sigma$. Then $\mathbf{K}_{d, \rho}$ annihilates $f_{\sigma}$ for $d>2(g-1) \operatorname{dim} \rho$ unless the restriction of $\rho$ to ${ }^{\lambda} H$ has non-zero invariants or coinvariants.

We expect that the representation $\rho \circ \sigma$ is semi-simple, and hence its invariants and coinvariants are isomorphic. We will assume that this is the case in what follows.

4.9. Proof of Lemma 1. In order to prove the lemma, we recall the GrothendieckLefschetz formula for the $L$-function.

Suppose we are given an unramified $n$-dimensional $\ell$-adic representation of $W(F)$. We attach to it an $\ell$-adic locally constant sheaf (local system) $\mathcal{L}$ on $X$.

Let $X^{(d)}=X^{d} / S_{d}$ be the $d$ th symmetric power of $X$. This is a smooth algebraic variety defined over $k$, whose $k$-points are effective divisors on $X$ of degree $d$. We define a sheaf on $X^{(d)}$, denoted by $\mathcal{L}^{(d)}$ and called the $d$ th symmetric power of $\mathcal{L}$, as follows:

$$
\mathcal{L}^{(d)}=\left(\pi_{*}^{d}\left(\mathcal{L}^{\bigotimes d}\right)\right)^{S^{(d)}}
$$

where $\pi^{d}: X^{d} \rightarrow X^{(d)}$ is the natural projection. The stalks of $\mathcal{L}^{(d)}$ are easy to describe: they are tensor products of symmetric powers of the stalks of $\mathcal{L}$. The stalk $\mathcal{L}_{d, D}$ at a divisor $D=\sum_{i} n_{i}\left[x_{i}\right]$ is

$$
\mathcal{L}_{D}^{(d)}=\bigotimes_{i} S^{n_{i}}\left(\mathcal{L}_{x_{i}}\right)
$$

where $S^{n_{i}}\left(\mathcal{L}_{x_{i}}\right)$ is the $n_{i}$-th symmetric power of the vector space $\mathcal{L}_{x_{i}}$. In particular, the dimensions of the stalks are not the same, unless $n=1$. (In the case when $n=1$, the sheaf $\mathcal{L}^{(d)}$ is in fact a rank 1 local system on $X^{(d)}$.) For all $n, \mathcal{L}^{(d)}$ is actually a perverse sheaf on $X^{(d)}$ (up to cohomological shift), which is irreducible if and only if $\mathcal{L}$ is irreducible.

Now observe that

$$
\operatorname{Tr}\left(\operatorname{Fr}_{D}, \mathcal{L}_{D}^{(d)}\right)=\prod_{i} \operatorname{Tr}\left(\sigma\left(\operatorname{Fr}_{x_{i}}\right), \rho^{\left(n_{i}\right)}\right), \quad D=\sum_{i} n_{i}\left[x_{i}\right]
$$

where $\operatorname{Fr}_{D}$ is the Frobenius automorphism corresponding to the $k$-point $D$ of $X^{(d)}$ and $\mathcal{L}_{D}$ is the stalk of $\mathcal{L}_{d}$ at $D$.

By the Lefschetz trace formula (see, e.g., [M] [FK]), the trace of the Frobenius on the étale cohomology of an $\ell$-adic sheaf is equal to the sum of the traces on the stalks at the $\mathbb{F}_{q}$-points:

$$
\operatorname{Tr}\left(\operatorname{Fr}, H^{\bullet}\left(X^{(d)}, \mathcal{L}^{(d)}\right)\right)=\sum_{D \in X^{(d)}\left(\mathbb{F}_{q}\right)} \operatorname{Tr}\left(\operatorname{Fr}_{D}, \mathcal{L}_{D}^{(d)}\right),
$$


By formula (4.19), the right-hand side of (4.20) is $l_{d, \rho}$ given by formula (4.14), and also the $q^{-d s}$-coefficient of the $L$-function $L\left(\sigma, \rho_{\mathrm{def}}, q^{-s}\right)$ of the representation $\sigma: W(F) \rightarrow G L_{n}$ associated to $\mathcal{L}$ and the defining $n$-dimensional representation $\rho_{\text {def }}$ of $G L_{n}$.

Let us compute the cohomology of $X^{(d)}$ with coefficients in $\mathcal{L}^{(d)}$. By the Künneth formula, we have

$$
H^{\bullet}\left(X^{(d)}, \mathcal{L}^{(d)}\right)=\left(H^{\bullet}\left(X^{d}, \mathcal{L}^{\bigotimes d}\right)\right)^{S_{d}}=\left(H^{\bullet}(X, \mathcal{L})^{\otimes d}\right)^{S_{d}}
$$

where the action of the symmetric group $S_{d}$ on the cohomology is as follows: it acts by the ordinary transpositions on the even cohomology and by signed transpositions on the odd cohomology. Thus, we find that

$$
\begin{aligned}
H^{\bullet}\left(X^{(d)}, \mathcal{L}^{(d)}\right) & \\
= & \bigoplus_{d_{0}+d_{1}+d_{2}=d} S^{d_{0}}\left(H^{0}(X, \mathcal{L})\right) \otimes \Lambda^{d_{1}}\left(H^{1}(X, \mathcal{L})\right) \otimes S^{d_{2}}\left(H^{2}(X, \mathcal{L})\right) .
\end{aligned}
$$

The cohomological grading is computed according to the rule that $d_{0}$ does not contribute to cohomological degree, $d_{1}$ contributes $d_{1}$, and $d_{2}$ contributes $2 d_{2}$. In addition, we have to take into account the cohomological grading on $\mathcal{L}$.

Formulas (4.20) and (4.21) give us the following expression for the generating function of the right-hand side of (4.20):

$$
\begin{aligned}
\sum_{d \geq 0} t^{d} \sum_{D \in X^{(d)}\left(\mathbb{F}_{q}\right)} \operatorname{Tr}\left(\operatorname{Fr}_{D}, \mathcal{L}_{D}\right) & \\
= & \frac{\operatorname{det}\left(1-t \operatorname{Fr}, H^{1}(X, \mathcal{L})\right)}{\operatorname{det}\left(1-t \operatorname{Fr}, H^{0}(X, \mathcal{L})\right) \operatorname{det}\left(1-t \operatorname{Fr}, H^{2}(X, \mathcal{L})\right)} .
\end{aligned}
$$

This is the Grothendieck-Lefschetz formula for the $L$-function $L\left(\sigma, \rho_{\text {def }}, t\right)$.

Now let $\mathcal{L}_{\rho \circ \sigma}$ be the $\ell$-adic local system corresponding to the representation $\rho \circ \sigma$ of $W(F)$. Then the trace of the Frobenius on the right-hand side of (4.21) gives us the eigenvalue $l_{d, \rho}$ of $\mathbf{K}_{d, \rho}$ on $f_{\sigma}$.

If $\rho \circ \sigma$ has zero spaces of invariants and coinvariants, then

$$
H^{0}\left(X, \mathcal{L}_{\rho \circ \sigma}\right)=H^{2}\left(X, \mathcal{L}_{\rho \circ \sigma}\right)=0
$$

and $\operatorname{dim} H^{1}\left(X, \mathcal{L}_{\rho \circ \sigma}\right)=(2 g-2) \operatorname{dim} \rho$ (since it is then equal to the Euler characteristic of the constant local system of $\operatorname{rank} \operatorname{dim} \rho$ on $X)$. Hence we obtain that

$$
H^{\bullet}\left(X^{(d)}, \mathcal{L}_{\rho \circ \sigma}^{(d)}\right) \simeq \Lambda^{d}\left(H^{1}\left(X, \mathcal{L}_{\rho \circ \sigma}\right)\right),
$$

and the $L$-function $L\left(\sigma, \rho, q^{-s}\right)$ is a polynomial in $q^{-s}$ of degree $2(g-1) \operatorname{dim} \rho$. Lemma 1 then follows from formula (4.16).

4.10. Decomposition of the trace formula. The goal of the program outlined in [FLN] (see also [L2, L4, L5]) is to use the trace formula to prove the existence of the functoriality transfers corresponding to the homomorphisms $\phi$ given by formula (4.4) in Section 4.3. The basic idea is to apply the trace formula to the operators $\mathbf{K}_{d, \rho}$ and use Lemma 1. Here is a more precise description.

As the first step, we need to remove from the trace formula the contributions of the non-tempered representations (those correspond to the transfer associated to the homomorphisms $\phi$ whose restriction to $\mathrm{SL}_{2}$ is non-trivial), because these terms dominate the trace formula (see the calculation below). Then we want to use 
Lemma 1 to isolate in the trace of $\mathbf{K}_{d, \rho}$ with $d>(2 g-2) \operatorname{dim} \rho$ the terms corresponding to the automorphic representations of $G\left(\mathbb{A}_{F}\right)$ that come by functoriality from the groups $H$ satisfying the $(G, \rho)$ property.

Recall from Section 4.3 that to each Arthur parameter $\sigma$ we attach the group ${ }^{\lambda} H={ }^{\lambda} H_{\sigma}$ which is the Zariski closure of the image of $W(F)$ in ${ }^{\lambda} G_{\sigma}$ under $\sigma$. According to Lemma 1 and Corollary 2, for large enough $d$ the operator $\mathbf{K}_{d, \rho}$ acts non-trivially only on those automorphic representations of $G\left(\mathbb{A}_{F}\right)$ which correspond to $\sigma$ such that $\rho \circ \sigma$ has non-zero invariants. This happens if and only if the restriction of $\rho$ to ${ }^{\lambda} H_{\sigma}$ has non-zero invariants; in other words, if and only if ${ }^{\lambda} H$ satisfies property $(G, \rho)$.

Thus, assuming that Arthur's conjectures are true, we obtain that the trace of $\mathbf{K}_{d, \rho}$ decomposes as a double sum: first, over different homomorphisms

$$
\varphi: \mathrm{SL}_{2} \rightarrow{ }^{L} G,
$$

and second, for a given $\varphi$, over the subgroups ${ }^{\lambda} H$ of the centralizer ${ }^{\lambda} G_{\varphi}$ of $\varphi$ having non-zero invariants in $\rho$,

$$
\operatorname{Tr} \mathbf{K}_{d, \rho}=\sum_{\varphi} \sum_{\lambda_{H \subset{ }^{\lambda} G_{\varphi}}} \Phi_{\varphi,{ }^{\lambda} H}
$$

Here $\Phi_{\varphi, \lambda H}$ is the trace over the automorphic representations of $G\left(\mathbb{A}_{F}\right)$ which come from the transfer of tempered (hadronic) representations of $H\left(\mathbb{A}_{F}\right)$ with respect to homomorphisms $\phi$, given by (4.4), such that $\left.\phi\right|_{\mathrm{SL}_{2}}=\varphi$.

A precise formula for these eigenvalues of $\mathbf{K}_{d, \rho}$ is complicated in general, but we can compute its asymptotics as $d \rightarrow \infty$.

Suppose first that $\varphi$ is trivial, so we are dealing with the tempered representations. If we divide $\mathbf{K}_{d, \rho}$ by $q^{d}$, then the asymptotics will be very simple:

$$
q^{-d}\left(\operatorname{Tr} \mathbf{K}_{d, \rho}\right)_{\text {temp }} \sim \sum_{\lambda} \sum_{\lambda^{L} \subset^{L} G} N_{\sigma}\left(\begin{array}{c}
d+m_{\sigma}(\rho)-1 \\
m_{\sigma}(\rho)-1
\end{array}\right),
$$

where $N_{\sigma}$ is the multiplicity of automorphic representations in the corresponding $L$-packet.

Indeed, the highest power of $q$ comes from the highest cohomology, which in this case is

$$
H^{2 d}\left(X^{(d)}, \mathcal{L}_{\rho \circ \sigma}^{(d)}\right)=\operatorname{Sym}^{d}\left(H^{2}(X, \rho \circ \sigma)\right)
$$

$\left(d_{0}=0, d_{1}=0\right.$, and $d_{2}=d$ in the notation of formula (4.21) $)$. We have

$$
\operatorname{dim} H^{2}(X, \rho \circ \sigma)=m_{\sigma}(\rho),
$$

the multiplicity of the trivial representation in $\rho \circ \sigma$ (we are assuming here again that this trivial representation splits off as a direct summand in $\rho \circ \sigma$ ), and

$$
\operatorname{dim} \operatorname{Sym}^{d}\left(H^{2}\left(X, \mathcal{L}_{\rho \circ \sigma}\right)\right)=\left(\begin{array}{c}
d+m_{\sigma}(\rho)-1 \\
m_{\sigma}(\rho)-1
\end{array}\right) .
$$

Thus, as a function of $q^{d}$, the eigenvalues of $q^{-d} \mathbf{K}_{d, \rho}$ on the tempered representations grow as $O(1)$ when $d \rightarrow \infty$.

For the non-tempered representations corresponding to non-trivial $\varphi: \mathrm{SL}_{2} \rightarrow{ }^{L} G$, they grow as a higher power of $q^{d}$. For instance, the eigenvalue of $q^{-d} \mathbf{K}_{d, \rho}$ corresponding to the trivial representation of $G\left(\mathbb{A}_{F}\right)$ (for which $\varphi$ is a principal embedding) grows as $O\left(q^{d(\rho, \mu)}\right)$, where $\mu$ is the highest weight of $\rho$ (see the calculation in Section 5.2 below). In general, it grows as $O\left(q^{d a}\right)$, where $2 a$ is the maximal 
possible highest weight of the image of $\mathrm{SL}_{2} \subset{ }^{L} G$ under $\varphi$ acting on $\rho$. Thus, we see that the asymptotics of the non-tempered representations dominates that of tempered representations. This is why we wish to remove the contribution of the non-tempered representations first.

Note that if $\varphi$ is non-trivial, then the rank of ${ }^{\lambda} G_{\varphi}$ is less than that of ${ }^{L} G$. As explained in [FLN], we would like to use induction on the rank of ${ }^{L} G$ to isolate and get rid of the terms in (4.23) with non-trivial $\varphi$. In [FLN] it was shown how to isolate the contribution of the trivial representation of $G\left(\mathbb{A}_{F}\right)$ for which $\varphi$ is the principal embedding (it is, along with all other one-dimensional representations of $G\left(\mathbb{A}_{F}\right)$, the most non-tempered).

If we can do the same with other non-tempered contributions, then we will be left with the terms $\Phi_{\text {triv },{ }^{\lambda} H}$ in (4.23) corresponding to the tempered representations of $G\left(\mathbb{A}_{F}\right)$. Denote their sum by $\left(\operatorname{Tr} \mathbf{K}_{d, \rho}\right)_{\text {temp. }}$. We try to decompose it as a sum over ${ }^{\lambda} H$ :

$$
\left(\operatorname{Tr} \mathbf{K}_{d, \rho}\right)_{\text {temp }}=\sum_{\lambda \subset^{L}{ }^{G}}\left(\operatorname{Tr} \mathbf{K}_{d, \rho_{H}}^{H}\right)_{\text {temp }} .
$$

Here the sum should be over all possible ${ }^{\lambda} H \subset{ }^{L} G$ such that ${ }^{\lambda} H$ has non-zero invariant vectors in $\rho$, and $\mathbf{K}_{d, \rho_{H}}^{H}$ is the operator corresponding to $\rho_{H}=\left.\rho\right|_{\lambda_{H}}$ for the group $H\left(\mathbb{A}_{F}\right)$ (note that different groups $H$ may correspond to the same ${ }^{\lambda} H$ ).

The ultimate goal is to prove formula (4.26) by comparing the orbital sides of the trace formula for the operators $\mathbf{K}_{d, \rho}$ and $\mathbf{K}_{d, \rho_{H}}^{H}$. Of course, for any given $\rho$, the right-hand side of formula (4.26) will contain contributions from different groups $H$. However, because we have two parameters, $\rho$ and $d$ (sufficiently large), we expect to be able to separate the contributions of different groups by taking linear combinations of these formulas with different $\rho$ and $d$. In the case of $G=G L_{2}$, this is explained in [L2].

There are many subtleties involved in formulas (4.23) and (4.26). As we mentioned above, ${ }^{\lambda} H$ may not be itself a Langlands dual group, but it can be enlarged to one. There may exist more than one conjugacy class of ${ }^{\lambda} H$ assigned to a given automorphic representation; this is expected to be related to the multiplicities of automorphic representations. Also, comparisons of trace formulas should always be understood as comparisons of their stabilized versions. Therefore the traces in (4.26) should be replaced by the corresponding stable traces

The upshot is that we want to establish formula (4.26) by proving identities between the corresponding orbital integrals. In the case of $G=\mathrm{SL}_{2}$ the first steps have been made in [L4, to which we refer the reader for more details.

One powerful tool that we hope to employ is the geometrization of these orbital integrals. We discuss this in the next section.

\section{Geometrization of the orbital part of the trace formula}

Our goal is to construct, in the case that the curve $X$ is defined over a finite field $\mathbb{F}_{q}$, a vector space with a natural action of $\operatorname{Gal}\left(\overline{\mathbb{F}}_{q} / \mathbb{F}_{q}\right)$ such that the trace of the Frobenius automorphism is equal to the right-hand side of the trace formula (4.10). We hope that this construction will help us to prove the decompositions (4.23) and (4.26) on the orbital side of the trace formula. Another important aspect of the construction is that this vector space will be defined in such a way that it will also make sense if the curve $X$ is over $\mathbb{C}$. In this section we outline this construction 
following $[\mathrm{FN}]$. (We will discuss the geometrization of the spectral side of the trace formula in Section 6.)

This section is organized as follows. In Section 5.1 we describe the geometric analogues of the Arthur parameters. These are certain complexes of local systems on the curve $X$. Then in Section 5.2 we construct the sheaf $\mathcal{K}_{d, \rho}$ such that the corresponding function is the kernel $K_{d, \rho}$ from the previous section. We define the corresponding functor $\mathbb{K}_{d, \rho}$ acting on the category of sheaves on Bun ${ }_{G}$ in Section 5.3 . In order to geometrize the orbital part of the trace formula, we need to restrict $\mathcal{K}_{d, \rho}$ to the diagonal and take the cohomology. This is explained in Section 5.4. We interpret the resulting vector space as the cohomology of a certain moduli stack defined in Sections 5.5 5.6 which we call the moduli of $G$-pairs. We compare it to the Hitchin moduli stack in Section 5.7 and define an analogue of the Hitchin map in Section 5.8. Most of this is taken from [FN]. In Section 5.9 we discuss in detail the example of $G=G L_{2}$. Finally, we present some of the conjectures of [FN] in Section 5.10 .

5.1. Geometric Arthur parameters. First, we discuss geometric analogues of the Arthur parameters (in the unramified case).

Let $\rho$ be a representation of ${ }^{L} G$ on a finite-dimensional vector space $V$. Then we obtain a representation $\rho \circ \sigma$ of $\mathrm{SL}_{2} \times W(F)$ on $V$. The standard torus of $\mathrm{SL}_{2} \subset \mathrm{SL}_{2} \times W(F)$ defines a $\mathbb{Z}$-grading on $\rho \circ \sigma$ :

$$
\rho \circ \sigma=\bigoplus_{i \in \mathbb{Z}}(\rho \circ \sigma)_{i}
$$

where each $\rho \circ \sigma_{i}$ is a continuous representation of $W(F)$. Assume that each of them is unramified. Then it gives rise to an $\ell$-adic local system $\mathcal{L}_{(\rho \circ \sigma)}$ on $X$. Now we define $\mathcal{L}_{\rho \circ \sigma}$ to be the following complex of local systems on $X$ with the trivial differential:

$$
\mathcal{L}_{\rho \circ \sigma}=\bigoplus_{i \in \mathbb{Z}} \mathcal{L}_{(\rho \circ \sigma)_{i}}[-i]
$$

We generalize the notion of Hecke eigensheaf by allowing its eigenvalue to be an Arthur parameter $\sigma$, as in (4.2), by saying that we have a collection of isomorphisms

$$
\mathbb{H}_{\rho}(\mathcal{F}) \simeq \mathcal{L}_{\rho \circ \sigma} \otimes \mathcal{F},
$$

for $\rho \in \operatorname{Rep}{ }^{L} G$, compatible with respect to the structures of tensor categories on both sides.

As an example, consider the constant sheaf on $\operatorname{Bun}_{G}, \mathcal{F}_{0}=\underline{\mathbb{Q}_{\ell}} \mid \operatorname{Bun}_{G}$. This is the geometric analogue of the trivial representation of $G\left(\mathbb{A}_{F}\right.$. Assume that $G$ is split, and let $\rho=\rho_{\mu} \otimes \rho_{0}$, where $\rho_{\mu}$ is the irreducible representation of highest weight $\mu, \rho_{0}$ is the trivial representation of $\Gamma$. In this case $\mathcal{K}_{\rho}=\mathcal{K}_{\mu}$ is the intersection cohomology complex of $\mathcal{H}_{\mu}$ shifted by $-\operatorname{dim}\left(X \times \operatorname{Bun}_{G}\right)$.

Let us apply the Hecke functor $\mathbb{H}_{\mu, x}$ to $\mathcal{F}_{0}$. For any $G$-principal bundle $E$, the fiber of $p^{-1}(E) \cap \mathcal{H}_{x}$ is isomorphic to $\mathrm{Gr}_{x}$, once we have chosen a trivialization of $E$ on the formal disc $D_{x}$. Thus the fiber of $\mathbb{H}_{\rho, x}\left(\mathcal{F}_{0}\right)$ at $E$ is isomorphic to

$$
\mathbb{H}_{\rho, x}(\mathcal{F})_{E}=H^{\bullet}\left(\overline{\mathrm{Gr}}_{\mu}, \mathrm{IC}\left(\overline{\mathrm{Gr}}_{\mu}\right)\right) .
$$

This isomorphism does not depend on the choice of the trivialization of $E$ on $D_{x}$, so we obtain that

$$
\mathbb{H}_{\rho, x}\left(\mathcal{F}_{0}\right) \simeq H^{\bullet}\left(\overline{\mathrm{Gr}}_{\mu}, \mathrm{IC}\left(\overline{\mathrm{Gr}}_{\mu}\right)\right) \otimes \mathcal{F}_{0}
$$


By the geometric Satake correspondence [MV],

$$
H^{\bullet}\left(\overline{\mathrm{Gr}}_{\mu}, \mathrm{IC}\left(\overline{\mathrm{Gr}}_{\mu}\right)\right) \simeq \rho_{\mu}^{\mathrm{gr}},
$$

a complex of vector spaces, which is isomorphic to the representation $\rho_{\mu}$ with the cohomological grading corresponding to the principal grading on $\rho_{\mu}$. One can show that as we vary $x$, the eigenvalue of the Hecke functor $\mathbb{H}_{\rho}$ is the complex $\rho_{\mu}^{\mathrm{gr}} \otimes \mathcal{L}_{0}$, where $\mathcal{L}_{0}$ is the trivial local system on $X$. In other words, it is the local system $\mathcal{L}_{\rho \circ \sigma_{0}}$ as defined above, where $\sigma_{0}: W(F) \times \mathrm{SL}_{2} \rightarrow{ }^{L} G$ is trivial on $W(F)$ and is the principal embedding on $\mathrm{SL}_{2}$. We conclude that the constant sheaf on $\operatorname{Bun}_{G}$ is a Hecke eigensheaf with the eigenvalue $\sigma_{0}$. This is in agreement with the fact that $\sigma_{0}$ is the Arthur parameter of the trivial automorphic representation of $G\left(\mathbb{A}_{F}\right)$.

For example, if $\rho_{\mu}$ is the defining representation of $G L_{n}$, then the corresponding Schubert variety is $\mathbb{P}^{n-1}$, and we obtain its cohomology shifted by $(n-1) / 2$, because the intersection cohomology sheaf $\operatorname{IC}\left(\overline{\mathrm{Gr}}_{\mu}\right)$ is the constant sheaf placed in cohomological degree $-(n-1)$, that is

$$
H^{\bullet}\left(\overline{\mathrm{Gr}}_{\mu}, \operatorname{IC}\left(\overline{\operatorname{Gr}}_{\mu}\right)\right)=\theta^{(n-1) / 2} \oplus \theta^{(n-3) / 2} \oplus \cdots \oplus \theta^{-(n-1) / 2},
$$

where $\theta^{1 / 2}=\mathbb{Q}_{\ell}[-1](-1 / 2)$. This agrees with the fact that the principal grading takes values $(n-1) / 2, \ldots,-(n-1) / 2$ on the defining representation of $G L_{n}$, and each of the corresponding homogeneous components is one dimensional.

5.2. The sheaf $\mathcal{K}_{d, \rho}$. Next, we define a geometric analogue of the operator $\mathbf{K}_{d, \rho}$. Recall that $\mathbf{K}_{d, \rho}$ is defined as the integral operator with the kernel $K_{d, \rho}$ given by formula (4.11). It is a function on the set of $\mathbb{F}_{q}$-points of the moduli stack Bun ${ }_{G}$ of $G$-bundles on $X$. Hence, according to the Grothendieck philosophy, we need to replace $K_{d, \rho}$ by an $\ell$-adic sheaf $\mathcal{K}_{d, \rho}$ on $\operatorname{Bun}_{G} \times \operatorname{Bun}_{G}$, whose trace of Frobenius function is $K_{d, \rho}$. We then define $\mathbb{K}_{d, \rho}$ as the corresponding integral transform functor acting on the derived category of $\ell$-adic sheaves on $\operatorname{Bun}_{G}$. The construction of this sheaf will work also if $X$ is defined over $\mathbb{C}$, in which case $\mathbb{K}_{d, \rho}$ will be a functor on the derived category of $\mathcal{D}$-modules on $\operatorname{Bun}_{G}$.

Since $K_{d, \rho}$ is built from the kernels $K_{\rho^{(n)}{ }_{x}}$ of the Hecke operators according to formula (4.11), we need to perform the same construction with the sheaves $\mathcal{K}_{\rho^{(n)}, x}$ defined in Section 3.4, which are the geometric counterparts of the $K_{\rho^{(n)}, x}$.

Introduce the algebraic stack $\mathcal{H}_{d}$ over the field $k$ (which is either a finite field or $\mathbb{C})$ that classifies the data

$$
\left(D, E, E^{\prime}, \phi\right)
$$

where

$$
D=\sum_{i=1}^{r} n_{i}\left[x_{i}\right]
$$

is an effective divisor on our curve $X$ of degree $d$ (equivalently, a point of $X^{(d)}$ ), $E$ and $E^{\prime}$ are two principal $G$-bundles on $X$, and $\phi$ is an isomorphism between them over $X-\operatorname{supp}(D)$.

Let $\mathcal{H}_{d, \mu}$ be the closed substack of $\mathcal{H}_{d}$ that classifies the quadruples (5.2) as above, satisfying the condition $\operatorname{inv}_{x_{i}}\left(E, E^{\prime}\right) \leq n_{i} \mu$.

Consider the morphism

$$
\mathcal{H}_{d, \mu} \rightarrow X^{(d)} \times \operatorname{Bun}_{G}
$$


sending the quadruple (5.2) to $(D, E)$. Its fiber over a fixed $D=\sum_{i} n_{i}\left[x_{i}\right]$ and $E \in \mathrm{Bun}_{G}$ is isomorphic to the product

$$
\prod_{i=1}^{r} \overline{\operatorname{Gr}}_{\left[n_{i} \mu_{i}\right]} .
$$

Our sheaf $\mathcal{K}_{d, \rho}$ on $\mathcal{H}_{d, \mu}$ will have the property that its restriction to these fibers are isomorphic to

$$
\bigotimes_{i=1}^{d} \mathrm{IC}_{\rho^{\left(n_{i}\right)}},
$$

where $\overline{\mathrm{Gr}}_{\left[n_{i} \mu_{i}\right]}$ is the perverse sheaf on $\overline{\mathrm{Gr}}_{\left[n_{i} \mu_{i}\right]}$ corresponding to the representation $\rho^{\left(n_{i}\right)}$ (the $n_{i}$-th symmetric power of $\rho$ ) under the geometric Satake.

The precise definition of $\mathcal{K}_{d, \rho}$ is given in [FN], and here we give a less formal, but more conceptual, construction.

We will use the following result from the Appendix of [FGV2] that generalizes the geometric Satake correspondence to the case of moving points.

For any partition $\mathbf{d}=\left(d^{1}, \ldots, d^{k}\right)$ of $d$, consider the open subset $\stackrel{\circ}{X^{\mathbf{d}}}$ of $X^{\left(d^{1}\right)} \times$ $\cdots \times X^{\left(d^{k}\right)}$ consisting of $k$-tuples of divisors $\left(D_{1}, \ldots, D_{k}\right)$, such that $\operatorname{supp} D_{i} \cap$ $\operatorname{supp} D_{j}=\emptyset$, if $i \neq j$. Denote the map $\stackrel{\circ}{X}^{\mathbf{d}} \rightarrow X^{(d)}$ by $p_{\mathbf{d}}$. We introduce an abelian category $\mathcal{A}^{d}$ as follows. The objects of $\mathcal{A}^{d}$ are perverse sheaves $\mathcal{F}$ on $X^{(d)}$ equipped with a ${ }^{L} G$-action, together with the following extra structure: for each partition $\mathbf{d}$, the sheaf $p_{\mathbf{d}}^{*}(\mathcal{F})$ should carry an action of $k$ copies of ${ }^{L} G$, compatible with the original ${ }^{L} G$-action on $\mathcal{F}$ with respect to the diagonal embedding ${ }^{L} G \rightarrow\left({ }^{L} G\right)^{\times k}$. For different partitions, these actions should be compatible in the obvious sense. In addition, it is required that whenever $d^{i}=d^{j}, i \neq j$, the action of the $i$ th and $j$ th copies of ${ }^{L} G$ on $p_{\mathbf{d}}^{*}(\mathcal{F})$ should be intertwined by the corresponding natural $\mathbb{Z}_{2}$-action on $\stackrel{\circ}{X} \mathbf{d}$.

The claim of [FGV2] is that the category $\mathcal{A}^{d}$ is equivalent to a certain category of perverse sheaves on $\mathcal{H}_{d}$. For instance, if $d=1$, then the constant sheaf on $X^{(1)}=X$ with the stalk $\rho=\rho_{\mu}$, a finite-dimensional representation of ${ }^{L} G$, goes to a perverse sheaf on $\mathcal{H}_{1}$, whose restriction to each fiber of the projection $\mathcal{H}_{1} \rightarrow X \times$ Bun $_{G}$ (which is isomorphic to $\mathrm{Gr}$ ) is the perverse sheaf $\mathrm{IC}_{\mu}$.

More generally, for each representation $\rho$ of ${ }^{L} G$ we have an object $\rho^{(d)}$ of the category $\mathcal{A}^{d}$ defined as follows:

$$
\underline{\rho}^{(d)}=\left(\pi_{*}^{d}\left(\underline{\rho}^{\bigotimes d}\right)\right)^{S_{d}},
$$

where $\rho$ is the constant sheaf on $X$ with the stalk $\rho, \pi^{d}: X^{d} \rightarrow X^{(d)}$ is the natural projection, and $S_{d}$ is the symmetric group on $d$ letters. It is easy to see that it carries the structures from the above definition. Moreover, it is an irreducible object of the category $\mathcal{A}^{d}$ if $\rho$ is irreducible. Note that the stalk of $\underline{\rho}^{(d)}$ at the divisor $D \subset X^{(d)}$ is the tensor product

$$
\bigotimes_{i} \rho^{\left(n_{i}\right)}
$$

where $\rho^{(n)}$ denotes the $n$th symmetric power of $\rho$.

Now, define the sheaf $\mathcal{K}_{d, \mu}$ as the irreducible perverse sheaf on $\mathcal{H}_{d}$ corresponding to $\underline{\rho}^{(d)}$. Then its restriction to the fiber at $(D, \mathcal{M}) \in X^{(d)} \times \operatorname{Bun}_{G}$ for general $D$ 
is given by formula (5.5), and so the trace of Frobenius function corresponding to $\mathcal{K}_{d, \mu}$ is the function $K_{d, \mu}$ given by formula (4.11).

Note that the highest weights of the irreducible representations in the decomposition of $\rho^{(n)}$ are less than or equal to $n \mu$, and so the sheaf $\mathrm{IC}_{\mu}^{(n)}$ is supported in $\overline{\mathrm{Gr}}_{n \mu}$. Therefore $\mathcal{K}_{d, \mu}$ is supported on the closed substack $\mathcal{H}_{d, \mu}$ introduced above.

5.3. The functor $\mathbb{K}_{d, \rho}$. We now have a morphism

$$
\mathcal{H}_{d} \rightarrow X^{(d)} \times \operatorname{Bun}_{G} \times \operatorname{Bun}_{G}
$$

and a perverse sheaf $\mathcal{K}_{d, \rho}$ on $\mathcal{H}_{d}$ attached to any finite-dimensional representation $\rho$ of ${ }^{L} G$. Let us denote by $p_{d}$ and $p_{d}^{\prime}$ the two projections $\mathcal{H}_{d} \rightarrow$ Bun $G$ mapping the quadruple (5.2) to $E$ and $E^{\prime}$. We use the sheaf $\mathcal{K}_{d, \rho}$ to define an integral transform functor $\mathbb{K}_{d, \rho}$ on the derived category $D\left(\operatorname{Bun}_{G}\right)$ of $\ell$-adic sheaves on $\operatorname{Bun}_{G}$ by the formula

$$
\mathbb{K}_{d, \rho}(\mathcal{F})=p_{d !}\left(p_{d}^{\prime *}(\mathcal{F}) \otimes \mathcal{K}_{d, \rho}\right) .
$$

Now we compute the eigenvalues of the functors $\mathbb{K}_{d, \rho}$ on $\mathcal{F}_{\sigma}$. The following result, which is the geometrization of formulas (4.14) and (4.19) for the eigenvalues of $\mathbf{K}_{d, \rho}$ on $f_{\sigma}$, is [FLN, Lemma 2.6].

Lemma 3. If $\mathcal{F}=\mathcal{F}_{\sigma}$ is a Hecke eigensheaf with eigenvalue $\sigma$, then for every representation $\rho$ of ${ }^{L} G$ and every positive integer $d$ we have

$$
\mathbb{K}_{d, \rho}\left(\mathcal{F}_{\sigma}\right)=H^{\bullet}\left(X^{(d)}, \mathcal{L}_{\rho \circ \sigma}^{(d)}\right) \otimes \mathcal{F}_{\sigma} .
$$

Taking the trace of the Frobenius on the right-hand side of formula (5.7), we obtain the eigenvalues of $\mathbf{K}_{d, \rho}$ on the Hecke eigenfunctions $f_{\sigma}$ corresponding to both tempered and non-tempered automorphic representations.

As an example, we compute the action of $\mathbb{K}_{d, \rho}$ on the constant sheaf on $\operatorname{Bun}_{G}$ :

$$
\mathbb{K}_{d, \rho_{\mu}}\left(\underline{\mathbb{Q}_{\ell}}\right) \simeq H^{\bullet}\left(X^{(d)}, \mathcal{L}_{\rho_{\mu} \circ \sigma_{0}}^{(d)}\right) \otimes \underline{\mathbb{Q}_{\ell}},
$$

where $\rho_{\mu} \circ \sigma_{0}$ is the complex described in Section 5.1.

At the level of functions, we are multiplying the constant function on $\operatorname{Bun}_{G}\left(\mathbb{F}_{q}\right)$ (corresponding to the trivial representation of $G\left(\mathbb{A}_{F}\right)$ ) by

$$
\operatorname{Tr}\left(\operatorname{Fr}, H^{\bullet}\left(X^{(d)}, \mathcal{L}_{\rho_{\mu} \circ \sigma_{0}}^{(d)}\right)\right)=\prod_{i \in P\left(\rho_{\mu}\right)} \zeta(s-i)^{\operatorname{dim} \rho_{\mu, i}},
$$

where $P\left(\rho_{\mu}\right)$ is the set of possible values of the principal grading on $\rho_{\mu}$ and $\rho_{\mu, i}$ is the corresponding subspace of $\rho_{\mu}$.

For example, if $\rho_{\mu}$ is the defining representation of $G L_{n}$, then formula (5.8) reads

$$
\prod_{k=0}^{n-1} \zeta(s+k-(n-1) / 2)
$$

5.4. Geometrization of the orbital side. Now we are ready to construct a geometrization of the orbital side of the trace formula.

Let us form the Cartesian square

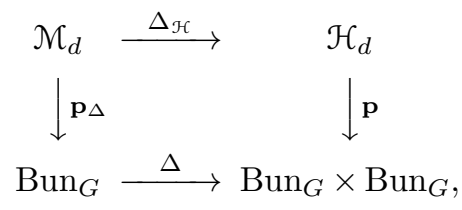


where $\Delta$ is the diagonal morphism. Thus, $\mathcal{M}_{d}$ is the fiber product of $X^{(d)} \times \operatorname{Bun}_{G}$ and $\mathcal{H}_{d}$ with respect to the two morphisms to $X^{(d)} \times \operatorname{Bun}_{G} \times \operatorname{Bun}_{G}$.

Let $\mathcal{K}=\mathcal{K}_{d, \rho}$ be a sheaf introduced in Section 5.2 and $\mathbb{K}=\mathbb{K}_{d, \rho}$ the corresponding functor on the derived category $D\left(\operatorname{Bun}_{G}\right)$ of sheaves on $\operatorname{Bun}_{G}$. The following discussion is applicable to more general functors that are compositions of $\mathbb{K}_{d, \rho}$ and Hecke functors $\mathbb{H}_{\rho_{i}, x_{i}}$ at finitely many points $x_{i} \in|X|$ and their kernels (see [FN]).

Let

$$
\overline{\mathcal{K}}=\mathbf{p}_{*}(\mathcal{K})
$$

(note that $\mathbf{p}$ is proper over the support of $\mathcal{K}$ ). This is a sheaf on $\operatorname{Bun}_{G} \times \operatorname{Bun}_{G}$ which is the kernel of the functor $\mathbb{K}$. Let $\bar{K}$ be the corresponding function on $\operatorname{Bun}_{G}(k) \times \operatorname{Bun}_{G}(k)$.

Recall that

$$
\operatorname{Bun}_{G}(k)=G(F) \backslash G\left(\mathbb{A}_{F}\right) / G\left(\mathcal{O}_{F}\right) .
$$

The right-hand side of (1.1) may be rewritten as

$$
\sum_{P \in \operatorname{Bun}_{G}(k)} \frac{1}{|\operatorname{Aut}(V)|} \bar{K}(P, P) .
$$

Using the Lefschetz formula for algebraic stacks developed by K. Behrend $\mathrm{Be}$ (see also $[\mathrm{BeDh}]$ ), we find that, formally, the alternating sum of the traces of the arithmetic Frobenius on the graded vector space

$$
H^{\bullet}\left(\operatorname{Bun}_{G}, \Delta^{!}(\overline{\mathcal{K}})\right)=H^{\bullet}\left(\operatorname{Bun}_{G}, \Delta^{!} \mathbf{p}_{*}(\overline{\mathcal{K}})\right)
$$

is equal (up to a power of $q$ ) to the sum (5.11). Therefore the vector space $H^{\bullet}\left(\operatorname{Bun}_{G}, \Delta^{!} \mathbf{p}_{*}(\overline{\mathcal{K}})\right)$ is a geometrization of the orbital (right-hand) side of the trace formula (1.1).

By base change,

$$
H^{\bullet}\left(\operatorname{Bun}_{G}, \Delta^{!} \mathbf{p}_{*}(\mathcal{K})\right)=H^{\bullet}\left(\operatorname{Bun}_{G}, \mathbf{p}_{\Delta *} \Delta_{\mathcal{H}}^{!}(\mathcal{K})\right)=H^{\bullet}\left(\mathcal{M}_{d}, \Delta_{\mathcal{H}}^{!}(\mathcal{K})\right) .
$$

We will use the space

$$
H^{\bullet}\left(\mathcal{M}_{d}, \Delta_{\mathcal{H}}^{!}(\mathcal{K})\right)
$$

as the geometrization of the orbital side.

Here is another way to express it: Let $\mathbb{D}$ be the Verdier duality on $\mathcal{H}_{d, \mu}$. It follows from the construction and the fact that $\mathbb{D}\left(\operatorname{IC}\left(\mathcal{H}_{\mu}\right)\right) \simeq \operatorname{IC}\left(\mathcal{H}_{\mu}\right)$ that

$$
\mathbb{D}\left(\mathcal{K}_{d, \rho}\right) \simeq \mathcal{K}_{d, \rho}\left[2\left(d+\operatorname{dim} \operatorname{Bun}_{G}\right)\right]\left(d+\operatorname{dim} \operatorname{Bun}_{G}\right) .
$$

Therefore, up to a shift and Tate twist, the last space in (5.13) is isomorphic to

$$
H^{\bullet}\left(\mathcal{M}_{d}, \mathbb{D}\left(\Delta_{\mathcal{H}}^{*}(\mathcal{K})\right)\right) \simeq H_{c}^{\bullet}\left(\mathcal{M}_{d}, \Delta_{\mathcal{H}}^{*}(\mathcal{K})\right)^{*},
$$

where $H_{c}^{\bullet}(Z, \mathcal{F})$ is understood as $f_{!}(\mathcal{F})$, where $f: Z \rightarrow$ pt. Here we use the results of Y. Laszlo and M. Olsson [LO on the six operations on $\ell$-adic sheaves on algebraic stacks and formula (5.15).

If $X$ is a curve over $\mathbb{C}$, then the vector space (5.14) still makes sense if we consider $\mathcal{K}$ as an object of either the derived category of constructible sheaves or of $\mathcal{D}$-modules on $\mathrm{Bun}_{G}$. 
5.5. The moduli stack of $G$-pairs. We now give a description of the stack $\mathcal{M}_{d}$ that is reminiscent and closely related to the Hitchin moduli stack of Higgs bundles on the curve $X$ [H1]. We will also conjecture that $\Delta_{\mathcal{H}}^{!}\left(\mathcal{K}_{d, \rho}\right)$ is a pure perverse sheaf on $\mathcal{M}_{d}$.

Recall that the sheaf $\mathcal{K}_{d, \rho}$ is supported on the substack $\mathcal{H}_{d, \mu}$ of $\mathcal{H}$. Let $\mathcal{M}_{d, \mu}$ be the fiber product of $X^{(d)} \times \operatorname{Bun}_{G}$ and $\mathcal{H}_{d, \mu}$ with respect to the two morphisms to $X^{(d)} \times \operatorname{Bun}_{G} \times \operatorname{Bun}_{G}$. In other words, we replace $\mathcal{H}_{d}$ by $\mathcal{H}_{d, \mu}$ in the upper-right corner of the diagram (5.10). The sheaf $\Delta_{\mathcal{H}}^{!}\left(\mathcal{K}_{d, \rho}\right)$ is supported on $\mathcal{M}_{d, \mu} \subset \mathcal{M}_{d}$, and hence the vector space (5.14) is equal to

$$
H^{\bullet}\left(\mathcal{M}_{d, \mu}, \Delta_{\mathcal{H}}^{!}\left(\mathcal{K}_{d, \rho}\right)\right) \text {. }
$$

In this section we show, following closely $[\mathrm{FN}]$, that the stack $\mathcal{M}_{d, \mu}$ has a different interpretation as a moduli stack of objects that are closely related to Higgs bundles. More precisely, we will have to define "group-like" versions of Higgs bundles (we call them $G$-pairs). The moduli spaces of (stable) Higgs bundles has been introduced by Hitchin [H1] (in characteristic 0) and the corresponding stack (in characteristic $p$ ) has been used in $\mathrm{N} 2$ in the proof of the fundamental lemma. In addition, there is an analogue $\mathcal{A}_{d, \mu}$ of the Hitchin base and a morphism $h_{d, \mu}: \mathcal{M}_{d, \mu} \rightarrow \mathcal{A}_{d, \mu}$ analogous to the Hitchin map. We hope that this Higgs bundle-like realization of $\mathcal{M}_{d, \mu}$ and the morphism $h_{d, \mu}$ can be used to derive the decompositions (4.23) and (4.26). In the next few sections we discuss this in more detail.

5.6. Definition of the moduli stack. Let us assume that $G$ is split over $X$ and $\mu$ is a fixed dominant coweight. The groupoid $\mathcal{M}_{d, \mu}(k)$ classifies the triples

$$
(D, E, \varphi) \text {, }
$$

where $D=\sum_{i} n_{i}\left[x_{i}\right] \in X^{(d)}$ is an effective divisor of degree $d, E$ is a principal $G$-bundle on a curve $X$, and $\varphi$ is a section of the adjoint group bundle

$$
\operatorname{Ad}(E)=E \underset{G}{\times} G
$$

(with $G$ acting on the right $G$ by the adjoint action) on $X-\operatorname{supp}(D)$, which satisfies the local conditions

$$
\operatorname{inv}_{x_{i}}(\varphi) \leq n_{i} \mu
$$

at $D$. Since we have defined $\mathcal{H}_{d, \mu}$ as the image of $\mathcal{H}_{\mu}^{d}$ in $\mathcal{H}_{d}$, it is not immediately clear how to make sense of these local conditions over an arbitrary base (instead of $\operatorname{Spec}(k))$. There is in fact a functorial description of $\mathcal{H}_{d, \mu}$ and of $\mathcal{M}_{d, \mu}$ that we will now explain.

We will assume that $G$ is semi-simple and simply-connected. The general case is not much more difficult. Let $\omega_{1}, \ldots, \omega_{r}$ denote the fundamental weights of $G$ and

$$
\rho_{\omega_{i}}: G \rightarrow \mathrm{GL}\left(V_{\omega_{i}}\right)
$$

the Weyl modules of highest weight $\omega_{i}$. Using the natural action of $G$ on $\operatorname{End}\left(V_{\omega_{i}}\right)$, we can attach to any $G$-principal bundle $E$ on $X$ the vector bundle

$$
\operatorname{End}_{\omega_{i}}(E)=E \underset{G}{\times} \operatorname{End}\left(V_{\omega_{i}}\right) .
$$

The section $\varphi$ of $\operatorname{Ad}(E)$ on $X-\operatorname{supp}(D)$ induces a section $\operatorname{End}_{\omega_{i}}(\varphi)$ of the vector bundle $\operatorname{End}_{\omega_{i}}(E)$ on $X-\operatorname{supp}(D)$. The local conditions (5.18) are equivalent to the 
property that for all $i, \operatorname{End}_{\omega_{i}}(\varphi)$ may be extended to a section

$$
\varphi_{i} \in \operatorname{End}_{\omega_{i}}(E) \otimes_{\mathcal{O}_{X}} \mathcal{O}_{X}\left(\left\langle\mu, \omega_{i}\right\rangle D\right) .
$$

Though the $\varphi_{i}$ determine $\varphi$, we will keep $\varphi$ in the notation for convenience.

Thus, we obtain a provisional functorial description of $\mathcal{M}_{d, \mu}$ as the stack classifying the data

$$
\left(D, E, \varphi, \varphi_{i}\right)
$$

with $D \in X^{(d)}, E \in \operatorname{Bun}_{G}, \varphi$ is a section of $\operatorname{Ad}(E)$ on $X-\operatorname{supp}(D)$, and $\varphi_{i}$ are sections of $\operatorname{End}_{\omega_{i}}(E) \otimes_{\mathcal{O}_{X}} \mathcal{O}_{X}\left(\left\langle\mu, \omega_{i}\right\rangle D\right)$ over $X$ such that

$$
\left.\varphi_{i}\right|_{X-\operatorname{supp}(D)}=\operatorname{End}_{\omega_{i}}(\varphi) .
$$

Sometimes it will be more convenient to package the data $\left(\varphi, \varphi_{i}\right)$ as a single object $\widetilde{\varphi}$ which has values in the closure of

$$
\left(t_{i} \rho_{\omega_{i}}(g)\right)_{i=1}^{r} \subset \prod_{i=1}^{r} \operatorname{End}\left(V_{\omega_{i}}\right) .
$$

where $g \in G$ and $t_{1}, \ldots, t_{r} \in \mathbb{G}_{m}$ are invertible scalars. This way Vinberg's semigroup [Vi] makes its appearance naturally in the description of $\mathcal{M}_{d, \mu}$.

5.7. Comparison with the Hitchin moduli stack. It is instructive to note that the stack $\mathcal{M}_{d, \mu}$ is very similar to the moduli stacks of Higgs bundles (defined originally by Hitchin [H1] and considered, in particular, in [N1, N2]). The latter stack - we will denote it by $\mathcal{N}_{D}$-also depends on the choice of an effective divisor

$$
D=\sum_{i} n_{i}\left[x_{i}\right]
$$

on $X$ and classifies pairs $(E, \phi)$, where $E$ is again a $G$-principal bundle on $X$ and $\phi$ is a section of the adjoint vector bundle

$$
\operatorname{ad}(E)=E \underset{G}{E} \mathfrak{g}
$$

(here $\mathfrak{g}=\operatorname{Lie}(G)$ ) defined on $X-\operatorname{supp}(D)$, which is allowed to have a pole of order at most $n_{i}$ at $x_{i}$. In other words,

$$
\phi \in H^{0}\left(X, \operatorname{ad}(E) \otimes \mathcal{O}_{X}(D)\right) .
$$

This $\phi$ is usually referred to as a Higgs field.

In both cases, we have a section which is regular almost everywhere, but at some (fixed, for now) points of the curve these sections are allowed to have singularities which are controlled by a divisor. In the first case we have a section $\varphi$ of adjoint group bundle $\operatorname{Ad}(E)$, and the divisor is $D \cdot \mu$, considered as an effective divisor with values in the lattice of integral weights of ${ }^{L} G$. In the second case we have a section $\phi$ of the adjoint Lie algebra bundle $\operatorname{ad}(E)$, and the divisor is just the ordinary effective divisor.

An important tool in the study of the moduli stack $\mathcal{N}_{D}$ is the Hitchin map [H2] from $\mathcal{N}_{D}$ to an affine space

$$
\mathcal{A}_{D} \simeq \bigoplus_{i} H^{0}\left(X, \mathcal{O}_{X}\left(\left(m_{i}+1\right) D\right)\right.
$$

where the $m_{i}$ 's are the exponents of $G$. It is obtained by, roughly speaking, picking the coefficients of the characteristic polynomial of the Higgs field $\phi$ (this is exactly so in the case of $G L_{n}$. but one constructs an obvious analogue of this morphism 
for a general reductive group $G$, using invariant polynomials on its Lie algebra). A point $a \in A_{E}$ then records a stable conjugacy class in $\mathfrak{g}(F)$, where $F$ is the function field, and the number of points in the fiber over $a$ is related to the corresponding orbital integrals in the Lie algebra setting (see [N1, N2]).

More precisely, $\mathcal{A}_{D}$ is the space of section of the bundle

$$
\mathfrak{t} / W \underset{\mathbb{G}_{m}}{\times} \mathcal{O}_{X}(D)^{\times}
$$

obtained by twisting $\mathfrak{t} / W=\operatorname{Spec}\left(k[\mathfrak{t}]^{W}\right)$, equipped with the $\mathbb{G}_{m}$-action inherited from $\mathfrak{t}$, by the $\mathbb{G}_{m}$-torsor $\mathcal{O}_{X}(D)^{\times}$on $X$ attached to the line bundle $\mathcal{O}_{X}(D)$. Recall that $k[\mathfrak{t} / W]$ is a polynomial algebra with homogeneous generators of degrees $d_{1}+1, \ldots, d_{r}+1$.

5.8. Analogue of the Hitchin map for $G$-pairs. In our present setting, we will have to replace $\mathfrak{t} / W$ by $T / W$. Recall that we are under the assumption that $G$ is semi-simple and simply connected. First, recall the isomorphism of algebras

$$
k[G]^{G}=k[T]^{W}=k[T / W] .
$$

It then follows from $\left[\mathrm{Bou}\right.$, Th. VI.3.1 and Ex. 1] that $k[G]^{G}$ is a polynomial algebra generated by the functions

$$
g \mapsto \operatorname{tr}\left(\rho_{\omega_{i}}(g)\right)
$$

where $\omega_{1}, \ldots, \omega_{r}$ are the fundamental weights of $G$.

For a fixed divisor $D$, the analogue of the Hitchin map for $\mathcal{M}_{d, \mu}(D)$ (the fiber of $\mathcal{M}_{d, \mu}$ over $\left.D\right)$ is the following map:

$$
\mathcal{M}_{d, \mu}(D) \rightarrow \bigoplus_{i=1}^{r} H^{0}\left(X, \mathcal{O}_{X}\left(\left\langle\mu, \omega_{i}\right\rangle D\right)\right)
$$

defined by attaching to $\left(D, E, \varphi, \varphi_{i}\right)$ the collection of traces

$$
\operatorname{tr}\left(\varphi_{i}\right) \in H^{0}\left(X, \mathcal{O}_{X}\left(\left\langle\mu, \omega_{i}\right\rangle D\right)\right) .
$$

By letting $D$ vary in $X^{(d)}$, we obtain a fibration

$$
h_{d, \mu}: \mathcal{M}_{d, \mu} \rightarrow \mathcal{A}_{d, \mu}
$$

where $\mathcal{A}_{d, \mu}$ is a vector bundle over $X^{(d)}$ with the fiber $\bigoplus_{i=1}^{r} H^{0}\left(X, \mathcal{O}_{X}\left(\left\langle\mu, \omega_{i}\right\rangle D\right)\right)$ over an effective divisor $D \in X^{(d)}$.

The morphism $h_{d, \mu}: \mathcal{M}_{d, \mu} \rightarrow \mathcal{A}_{d, \mu}$ is very similar to the Hitchin fibration.

Recall that according to Section 5.4 the cohomology of $\mathcal{M}_{d, \mu}$ with coefficients in $\Delta^{!}\left(\mathcal{K}_{d, \rho}\right)$ is a geometrization of the orbital side of the trace formula (1.1). This side of the trace formula may be written as a sum (4.9) of orbital integrals. To obtain a geometrization of an individual orbital integral appearing in this sum, we need to take the cohomology of the restriction of $\Delta^{!}\left(\mathcal{K}_{d, \rho}\right)$ to the corresponding fiber of the map $h_{d, \mu}$ (see [FN, Section 4.3]). Thus, the picture is very similar to that described in [N2] in the Lie algebra case.

In FLN we considered the trace formula in the general ramified setting. As in the above discussion, we obtained a useful interpretation of the stable conjugacy classes in $G(F)$ as $F$-points of the Hitchin base described above (in [FLN] we called it the Steinberg-Hitchin base). In particular, if the group $G$ is simply connected, this base has the structure of a vector space over $F$. The orbital side of the trace formula may therefore be written as the sum over points of this vector space, and we can apply the Poisson summation formula to it (actually, in order to do this we need 
to overcome several technical problems). We used this to isolate the contribution of the trivial representation to the trace formula, which appears as the contribution of the point 0 in the dual sum (see [FLN]). The question now is how to separate the contributions of other non-tempered automorphic representations of $G\left(\mathbb{A}_{F}\right)$.

5.9. Example of $G L_{2}$. Let us specialize now to the case when $G=G L_{2}$ and $\mu=(2,0)$. Here we need to make one adjustment of the general setup; namely, instead of

$$
G L_{2}(F) \backslash G L_{2}\left(\mathbb{A}_{F} / G L_{2}\left(\mathcal{O}_{F}\right),\right.
$$

which is a union of infinitely many components (hence non-compact), we will consider a compact quotient

$$
G L_{2}(F) a^{\mathbb{Z}} \backslash G L_{2}(\mathbb{A}) / G L_{2}(\mathcal{O}),
$$

where $a$ is an element in the center $Z\left(\mathbb{A}_{F}\right)$. We choose as $a$ the element equal to 1 at all $x \in X$, except for a fixed point that we denote by $\infty$. We set $a$ equal to a uniformizer $t_{\infty}$ at $\infty$. Thus, we do not allow ramification at $\infty$, but rather restrict ourselves to automorphic representations on which this $a$ acts trivially, in order to make sure that our integrals converge.

Since we now identify the rank-two bundle $\mathcal{M}$ with $\mathcal{M}(k[\infty])$ for all $k \in \mathbb{Z}$, which is an operation shifting the degree of the bundle by an even integer $2 k$, this stack has two connected components, corresponding to the degree of the bundle $\mathcal{M}$ modulo 2 .

We denote them by $\mathcal{H}_{d, \mu, \infty}^{\Delta, 0}$ and $\mathcal{H}_{d, \mu, \infty}^{\Delta, 1}$. Let us focus on the first one and set $\mu=(2,0)$ (so that $\rho$ is the symmetric square of the defining vector representation).

This $\mathcal{H}_{d,(2,0), \infty}^{\Delta, 0}$ is the moduli stack of the following data:

$$
(D, \mathcal{M}, s: \mathcal{M} \hookrightarrow \mathcal{M}(d[\infty])),
$$

where $D$ is an effective divisor on $X$ of degree $d$,

$$
D=\sum_{i=1}^{\ell} n_{i}\left[x_{i}\right],
$$

$\mathcal{M}$ is a rank-two vector bundle on $X$ of degree 0 , and $s$ is an injective map such that

$$
\mathcal{M}(d[\infty]) / s(\mathcal{M}) \simeq \bigoplus_{i} \mathcal{T}_{x_{i}}
$$

where the $\mathcal{T}_{x_{i}}$ are torsion sheaves supported at $x_{i}$. For instance, if all points in $D$ have multiplicity 1 , that is, $n_{i}=1$ for all $i$, then $\mathcal{T}_{x_{i}}=\mathcal{O}_{2 x_{i}}$ or $\mathcal{O}_{x_{i}} \oplus \mathcal{O}_{x_{i}}$. But if $n_{i}>1$, then it splits in one of the following ways:

$$
\mathcal{T}_{x_{i}}=\mathcal{O}_{k_{i} x_{i}} \oplus \mathcal{O}_{\left(2 n_{i}-k_{i}\right) x_{i}}, \quad k_{i}=0, \ldots, n_{i}
$$

(these correspond to the strata in the affine Grassmannian which lie in the closure of $\left.\mathrm{Gr}_{n_{i} \mu}\right)$.

What are the allowed values of $b_{1}, b_{2}$ ? By construction, $b_{2}$ is a section of $\mathcal{O}_{X}(2 d[\infty])$, whose divisor of zeros is $2 D$.

Lemma 4. Any such section $b_{2}$ of $\mathcal{O}_{X}(2 d[\infty])$ has the form $b_{2}=\eta^{2}$, where $\eta$ is a non-zero section of $\mathcal{L} \otimes \mathcal{O}_{X}(d[\infty])$, where $\mathcal{L}$ is a square root of the trivial line bundle (that is, $\mathcal{L}^{\otimes 2} \simeq \mathcal{O}_{X}$ ) having the divisor of zeros equal to $D$. 
Proof. Consider the equation $y^{2}=b_{2}$, where $y$ is a section of $\mathcal{O}_{X}(d[\infty])$. We interpret the solution as a curve in the total space of the line bundle $\mathcal{O}_{X}(d[\infty])$ over $X$, which is a ramified double cover $C$ of $X$, with the divisor of ramification equal to $D$ (indeed, away from the points of $D$ the value of $b_{2}$ is non-zero, hence there are two solutions for $y$, but at the points of $D$ we have $b_{2}=0$, so there is only one solution $y=0$ ). Moreover, near each point $x_{i}$ in $D$ (see formula (5.22) ) this curve $C$ is given by the equation $y^{2}=t^{2 n_{i}}$, and hence has two branches. Thus, the normalization of $C$ is an unramified double cover $\widetilde{C}$ of $X$. It gives rise to a square root $\mathcal{L}$ of $\mathcal{O}_{X} 4$

Clearly, the pull-back of $\mathcal{L}$ to $\widetilde{C}$ is canonically trivialized. Therefore a section of $\mathcal{L} \otimes \mathcal{O}_{X}(d[\infty])$ over $X$ is the same thing as a section of the pull-back of $\mathcal{O}_{X}(d[\infty])$ to $\widetilde{C}$ which is anti-invariant under the natural involution $\tau: \widetilde{C} \rightarrow \widetilde{C}$. But $y$ gives us just such a section (indeed, it assigns to each point $c$ of $\widetilde{C}$ a vector in the fiber of $\mathcal{O}_{X}(d[\infty])$ over the image of $c$ in $X$-namely, the image of $c$ in $C$, which is viewed as a point in the total space of $\mathcal{O}_{X}(d[\infty])$; if there are two points $c_{1}, c_{2}$ in $\widetilde{C}$ lying over the same point of $X$, then these vectors are obviously opposite to each other). Hence we obtain a section $\eta$ of $\mathcal{L} \otimes \mathcal{O}_{X}(d[\infty])$ over $X$. It follows that $\eta^{2}=b_{2}$.

For each square root $\mathcal{L}$ of the trivial line bundle on $X$, consider the map

$$
H^{0}\left(X, \mathcal{L} \otimes \mathcal{O}_{X}(d[\infty])\right)^{\times} \rightarrow H^{0}(X, \mathcal{O}(2 d[\infty]))
$$

sending

$$
\eta \mapsto \eta^{2}
$$

Denote the image by $B_{\mathcal{L}}$. It is isomorphic to the quotient of $H^{0}\left(X, \mathcal{L} \otimes \mathcal{O}_{X}(d[\infty])\right)^{\times}$ by the involution acting as $\eta \mapsto-\eta$.

We conclude that $b_{2}$ could be any point in

$$
\bigsqcup_{\mathcal{L}} B_{\mathcal{L}} \subset H^{0}(X, \mathcal{O}(2 d[\infty])) .
$$

Thus, we have described the possible values of $b_{2}$, which is the determinant of $\gamma$. This is a subset in a vector space, which has components labeled by $\mathcal{L}$, which we view as points of order two in $\operatorname{Jac}\left(\mathbb{F}_{q}\right)$. We denote the set of such points by $\mathrm{Jac}_{2}$ and its subset corresponding to non-trivial $\mathcal{L}$ by $\operatorname{Jac}_{2}^{\times}$.

What about $b_{1}$, which is the trace of $\gamma$ ? It is easy to see that $b_{1}$ may take an arbitrary value in $H^{0}\left(X, \mathcal{O}_{X}(d[\infty])\right)$. Thus, we find that the image of $\mathcal{H}_{d,(2,0), \infty}^{\Delta, 0}$ in

$$
H^{0}\left(X, \mathcal{O}_{X}(d[\infty])\right) \oplus H^{0}\left(X, \mathcal{O}_{X}(2 d[\infty])\right)
$$

under the map

$$
\gamma \mapsto(\operatorname{tr} \gamma, \operatorname{det} \gamma)=\left(b_{1}, b_{2}\right)
$$

is equal to

$$
H^{0}\left(X, \mathcal{O}_{X}(d[\infty])\right) \times\left(\bigsqcup_{\mathcal{L} \in \mathrm{Jac}_{2}} B_{\mathcal{L}}\right) .
$$

In other words, any $\gamma$ that has trace and determinant of this form corresponds to a point of $\mathcal{H}_{d,(2,0), \infty}^{\Delta, 0}$.

\footnotetext{
${ }^{4}$ Namely, we interpret $\widetilde{C}$ as a principal $\mathbb{Z}_{2}=\{ \pm 1\}$-bundle over $X$, and take the line bundle associated to the non-trivial one-dimensional representation of $\mathbb{Z}_{2}$.
} 
What does the fiber $F_{b_{1}, b_{2}}$ look like? Using the theory of Hitchin fibrations, we describe it as follows: consider the characteristic polynomial of $\gamma$ :

$$
z^{2}-b_{1} z+b_{2}=0
$$

This equation defines a curve $\widetilde{X}_{b_{1}, b_{2}}$ in the total space of the line bundle $\mathcal{O}_{X}(d[\infty])$ over $X$, which is called the spectral curve associated to $b_{1}, b_{2}$. If this curve is smooth, then the fiber $F_{b_{1}, b_{2}}$ is just the Jacobian of $\widetilde{X}_{b_{1}, b_{2}}$. What is the rank-two bundle $\mathcal{M}$ corresponding to a point of the Jacobian of $\widetilde{X}_{b_{1}, b_{2}}$ ? This point is a line bundle on $\widetilde{X}_{b_{1}, b_{2}}$. Take its push-forward to $X$. This is our $\mathcal{M}$. It comes equipped with a map $s: \mathcal{M} \rightarrow \mathcal{M}(d[\infty])$ whose trace and determinant are $b_{1}$ and $b_{2}$, respectively. The claim is that any point in $F_{b_{1}, b_{2}}$ may be obtained this way, as a push-forward of a line bundle on $\widetilde{X}_{b_{1}, b_{2}}$.

If $\widetilde{X}_{b_{1}, b_{2}}$ is singular, then $F_{b_{1}, b_{2}}$ is the compactification of the Jacobian of $\widetilde{X}_{b_{1}, b_{2}}$ known as the moduli space of torsion sheaves on $\widetilde{X}_{b_{1}, b_{2}}$ of generic rank one. It contains the Jacobian of $\widetilde{X}_{b_{1}, b_{2}}$ as an open dense subset.

5.10. General case. In FN, we outlined the geometric properties of the Hitchin fibration (used in [N2] to prove the fundamental lemma) that can be carried over to our new situation.

Our goal is to understand the cohomology (5.17). According to formula (5.15), up to a shift and Tate twist, it is isomorphic to the dual of the cohomology with compact support of $\Delta_{\mathcal{H}}^{*}\left(\mathcal{K}_{d, \rho}\right)$. The following conjecture seems to be necessary to have a chance to approach this cohomology using known methods (such as the decomposition theorem and Ngô's theorem about push-forwards of perverse sheaves [N2], which he used in the proof of the fundamental lemma). It probably tells us something important about the geometric trace formula and the categorical Langlands correspondence (the subjects discussed in Section 6), but it is not clear to me yet what it is.

Conjecture $5([\mathrm{FN}])$. The restriction to the diagonal $\Delta_{\mathcal{H}}^{*}\left(\mathcal{K}_{d, \rho}\right)$ is a pure perverse sheaf.

Assume that $G$ is semi-simple. As in CL, there exists a open substack $\mathcal{M}_{d, \mu}^{\mathrm{st}}$ of $\mathcal{M}_{d, \mu}$ that is proper over $\mathcal{A}_{d, \mu}$. This open substack depends on the choice of a stability condition. However, its cohomology should be independent of this choice. Moreover, there exists an open subset $\mathcal{A}_{d, \mu}^{\text {ani }}$ of $\mathcal{A}_{d, \mu}$ whose $\bar{k}$-points are the pairs $(D, b)$ such that as an element of $(T / W)\left(F \otimes_{k} \bar{k}\right), b$ corresponds to a regular semisimple and anisotropic conjugacy class in $G\left(F \otimes_{k} \bar{k}\right)$. The preimage $\mathcal{M}_{d, \mu}^{\text {ani }}$ of $\mathcal{A}_{d, \mu}^{\text {ani }}$ is contained in $\mathcal{M}_{d, \mu}^{\text {st }}$ for all stability conditions. In particular, the morphism $\mathcal{M}_{d, \mu}^{\text {ani }} \rightarrow$ $\mathcal{A}_{d, \mu}^{\text {ani }}$ is proper.

To compute the cohomology with compact support of $\Delta_{\mathcal{H}}^{*}\left(\mathcal{K}_{d, \rho}\right)$, we consider the sheaf $\left(h_{d, \mu}^{\mathrm{st}}\right) ! \Delta_{\mathcal{H}}^{*}\left(\mathcal{K}_{d, \rho}\right)=\left(h_{d, \mu}^{\mathrm{st}}\right)_{*} \Delta_{\mathcal{H}}^{*}\left(\mathcal{K}_{d, \rho}\right)$ on $\mathcal{A}_{d, \mu}^{\mathrm{st}}$ (recall that $h_{d, \mu}^{\mathrm{st}}$ is proper). By Deligne's purity theorem, Conjecture 5 implies that $\left(h_{d, \mu}^{\text {st }}\right)_{*} \Delta_{\mathcal{H}}^{*}\left(\mathcal{K}_{d, \rho}\right)$ is a pure complex. Hence, geometrically, it is isomorphic to a direct sum of shifted simple perverse sheaves.

In [FN], Ngô and I presented some conjectures describing the structure of $\left(h_{d, \mu}^{\text {st }}\right)_{*} \Delta_{\mathcal{H}}^{*}\left(\mathcal{K}_{d, \rho}\right)$, both in the general case and in the specific example of $G=S L_{2}$ and $H$ a twisted torus. 


\section{The geometric trace Formula}

In Section 5.4 we have interpreted geometrically the right-hand side of the trace formula (1.1). Now we turn to the left-hand (spectral) side. We will follow closely the exposition of $[\mathrm{FN}]$. We begin by rewriting the spectral side as a sum over the homomorphisms $W_{F} \times \mathrm{SL}_{2} \rightarrow{ }^{L} G$ in Section 6.1. It is tempting to interpret this sum using the Lefschetz fixed point formula. After explaining the difficulties in doing so directly in Section 6.2, we try a different approach in Section 6.3. Namely, we use the categorical Langlands correspondence of Section 3.10 to construct a vector space that should be isomorphic to the vector space of Section 5.4 that we proposed as the geometrization of the orbital side of the trace formula. This gives us the sought-after geometrization of the spectral side, and we declare the conjectural isomorphism between the two (implied by the categorical Langlands correspondence) as the geometric trace formula. In Section 6.4 we give an heuristic explanation why this vector space should indeed be viewed as the geometrization of the spectral side.

6.1. The left-hand side of the trace formula. We will assume that $G$ is split. As we discussed in Section 4.10, the $L$-packets of (unramified) irreducible automorphic representations should correspond to (unramified) homomorphisms $W_{F} \times \mathrm{SL}_{2} \rightarrow{ }^{L} G$. Assuming this conjecture and ignoring for the moment the contribution of the continuous spectrum, we may write the left-hand side of (4.7) as

$$
\operatorname{Tr} \mathbf{K}=\sum_{\sigma} m_{\sigma} N_{\sigma}
$$

where $\sigma$ runs over the unramified homomorphisms $W_{F} \times \mathrm{SL}_{2} \rightarrow{ }^{L} G, m_{\sigma}$ is the multiplicity of the irreducible automorphic representation unramified with respect to $G\left(\mathcal{O}_{F}\right)$ in the $L$-packet corresponding to $\sigma$, and $N_{\sigma}$ is the eigenvalue of the operator $\mathbf{K}$ on an unramified automorphic function $f_{\sigma}$ on $\operatorname{Bun}_{G}\left(\mathbb{F}_{q}\right)$ corresponding to a spherical vector in the representation

$$
\mathbf{K} \cdot f_{\sigma}=N_{\sigma} f_{\sigma} .
$$

Thus, recalling (5.11), the trace formula (4.7) becomes

$$
\sum_{\sigma: W_{F} \times \mathrm{SL}_{2} \rightarrow{ }^{L} G} m_{\sigma} N_{\sigma}=\sum_{P \in \mathrm{Bun}_{G}\left(\mathbb{F}_{q}\right)} \frac{1}{|\operatorname{Aut}(V)|} \bar{K}(P, P) .
$$

Consider, for example, the case of $\mathbf{K}=\mathbf{H}_{\rho, x}$, the Hecke operator corresponding to a representation $\rho$ of ${ }^{L} G$ and $x \in|X|$. Then, according to formula (4.3),

$$
N_{\sigma}=\operatorname{Tr}\left(\sigma\left(\left(\begin{array}{cc}
q_{x}^{1 / 2} & 0 \\
0 & q_{x}^{-1 / 2}
\end{array}\right) \times \operatorname{Fr}_{x}\right), \rho\right) .
$$

The $\mathbf{K}$ are generated by the Hecke operators $\mathbf{H}_{\rho, x}$. Therefore the eigenvalue $N_{\sigma}$ for such an operator $\mathbf{K}$ is expressed in terms of the traces of $\sigma\left(\mathrm{Fr}_{x}\right)$ on representations of ${ }^{L} G$.

6.2. Lefschetz fixed point formula interpretation. It is tempting to try to interpret the left-hand side of (6.2) as coming from the Lefschetz trace formula for the trace of the Frobenius on the cohomology of an $\ell$-adic sheaf on a moduli stack, whose set of $k$-points is the set of $\sigma$ 's. Unfortunately, such a stack does not exist if 
$k$ is a finite field $\mathbb{F}_{q}$ (or its algebraic closure). On the other hand, if $X$ is over $\mathbb{C}$, then there is an algebraic stack $\operatorname{Loc}_{L_{G}}$ of (de Rham) ${ }^{L} G$-local systems on $X$, that is, ${ }^{L} G$-bundles on $X$ with flat connection. But in this case there is no Frobenius acting on the cohomology whose trace would yield the desired number (the left-hand side of (6.2) ). Nevertheless, we will define a certain vector space (when $X$ is over $\mathbb{C}$ ), which we will declare to be a geometrization of the left-hand side of (6.2) (we will give an heuristic explanation for this in Section 6.4). We will then conjecture that this space is isomorphic to (5.14) - this will be the statement of the geometric trace formula that we propose in this paper.

Let us first consider the simplest case of the Hecke operator $\mathbf{K}=\mathbf{H}_{\rho, x}$. In this case the eigenvalue $N_{\sigma}$ is given by formula (6.3), which is essentially the trace of the Frobenius of $x$ on the vector space which is the stalk of the local system on $X$ corresponding to $\sigma$ and $\rho$. These vector spaces are fibers of a natural vector bundle on $X \times \operatorname{Loc}_{L}$ (when $X$ is defined over $\mathbb{C}$ ).

Indeed, we have a tautological ${ }^{L} G$-bundle $\mathcal{T}$ on $X \times \operatorname{Loc}_{L_{G}}$, whose restriction to $X \times \sigma$ is the ${ }^{L} G$-bundle on $X$ underlying $\sigma \in \operatorname{Loc}_{L_{G}}$. For a representation $\rho$ of ${ }^{L} G$, let $\mathcal{T}_{\rho}$ be the associated vector bundle on $X \times \operatorname{Loc}_{L_{G}}$. It then has a partial flat connection along $X$. Further, for each point $x \in|X|$, we denote by $\mathcal{T}_{x}$ and $\mathcal{T}_{\rho, x}$ the restrictions of $\mathcal{T}$ and $\mathcal{T}_{\rho}$, respectively, to $x \times \operatorname{Loc}_{L_{G}}$.

It is tempting to say that the geometrization of the left-hand side of (6.2) in the case $\mathbf{K}=\mathbf{H}_{\rho, x}$ is the cohomology

$$
H^{\bullet}\left(\operatorname{Loc}_{L}, \mathcal{T}_{\rho, x}\right) .
$$

However, this would only make sense if the vector bundle $\mathcal{T}_{\rho, x}$ carried a flat connection (i.e., a $\mathcal{D}$-module structure) and this cohomology was understood as the de Rham cohomology (which is the analogue of the étale cohomology of an $\ell$-adic sheaf that we now wish to imitate when $X$ is defined over $\mathbb{C}$ ).

Unfortunately, $\mathcal{T}_{\rho, x}$ does not carry any natural connection. Hence we search for another approach. Somewhat surprisingly, it is provided by the categorical Langlands correspondence of Section 3.7.

6.3. Geometrization of the spectral side of the trace formula. The categorical Langlands correspondence (3.10) should yield important information not only at the level of objects, but also at the level of morphisms. In particular, if we denote the equivalence going from left to right in the above diagram by $C$, then we obtain the isomorphisms

$$
\operatorname{RHom}_{D\left(\operatorname{Loc}_{L_{G}}\right)}\left(\mathcal{F}_{1}, \mathcal{F}_{2}\right) \simeq \operatorname{RHom}_{D\left(\operatorname{Bun}_{G}\right)}\left(C\left(\mathcal{F}_{1}\right), C\left(\mathcal{F}_{2}\right)\right)
$$

and

$$
\operatorname{RHom}\left(\mathbb{F}_{1}, \mathbb{F}_{2}\right) \simeq \operatorname{RHom}\left(C\left(\mathbb{F}_{1}\right), C\left(\mathbb{F}_{2}\right)\right),
$$

where $\mathbb{F}_{1}$ and $\mathbb{F}_{2}$ are arbitrary two functors acting on the category $D\left(\operatorname{Loc}_{L_{G}}\right)$. We will use the latter to produce the geometric trace formula.

Recall also the Wilson functors $\mathbb{W}_{\rho, x}$ from Section 3.7 and the compatibility (3.12) between the Wilson and Hecke functors under $C$.

We now construct a functor $\mathbb{W}_{d, \rho}$ on the category of $\mathcal{O}$-modules on $\operatorname{Loc}_{L_{G}}$ from the Wilson functors in the same way as we build the functor $\mathbb{K}_{d, \rho}$ from the Hecke functors. Informally speaking, it is the integral over all effective divisors 
$D=\sum_{i} n_{i}\left[x_{i}\right] \in X^{(d)}$ of $\prod_{i} \mathbb{W}_{\rho^{\left(n_{i}\right)}, x_{i}}$. The precise definition is given in $[\mathrm{FN}$, Section 5.3]. We have

$$
\mathbb{W}_{d, \rho}(\mathcal{F})=\mathcal{F}_{d, \rho} \otimes p_{2}^{*}(\mathcal{F}),
$$

where $\mathcal{F}_{d, \rho}$ is a certain $\mathcal{O}$-module on $\operatorname{Loc}_{L_{G}}$ explicitly defined in terms of the tautological vector bundles $\mathcal{T}_{\rho^{(n)}, x}$, and $p_{2}$ is the projection $X \times \operatorname{Loc}_{L_{G}} \rightarrow \operatorname{Loc}_{L_{G}}$.

The functor $\mathbb{W}_{\rho}$ may also be written as the integral transform functor corresponding to the $\mathcal{O}$-module $\Delta_{*}\left(\mathcal{F}_{d, \rho}\right)$ on $\operatorname{Loc}_{L_{G}} \times \operatorname{Loc}_{L_{G}}$, where $\Delta$ is the diagonal embedding of $\operatorname{Loc}_{L}$ :

$$
\left.\mathcal{F} \mapsto q_{*}\left(q^{\prime *}(\mathcal{F}) \otimes \Delta_{*}\left(\mathcal{F}_{d, \rho}\right)\right)\right),
$$

where $q$ and $q^{\prime}$ are the projections onto $X \times \operatorname{Loc}_{L_{G}}$ (the first factor) and $\operatorname{Loc}_{L_{G}}$ (the second factor), respectively.

Consider now the isomorphism (6.5) in the case $\mathbb{F}_{1}=\mathrm{Id}$ and $\mathbb{F}_{2}=\mathbb{W}_{d, \rho}$. It follows from the construction of the functors $\mathbb{W}_{d, \rho}$ and $\mathbb{H}_{d, \rho}$ and formula (3.12). Hence (6.5) gives us an isomorphism

$$
\mathrm{RHom}\left(\mathrm{Id}, \mathbb{W}_{d, \rho}\right) \simeq \mathrm{RHom}\left(\mathrm{Id}, \mathbb{K}_{d, \rho}\right) .
$$

We should also have an isomorphism of the RHoms's of the kernels defining these functors. On the right-hand side this is the RHom

$$
\operatorname{RHom}\left(\Delta_{!}(\underline{\mathcal{e}}), \overline{\mathcal{K}}_{d, \rho}\right),
$$

where $\underline{\mathcal{C}}$ is the constant sheaf on $\operatorname{Bun}_{G}$ and $\overline{\mathcal{K}}_{d, \rho}=\mathbf{p}_{*}\left(\mathcal{K}_{d, \rho}\right)$ (see Section 5.4) in the derived category of $\mathcal{D}$-modules on $\operatorname{Bun}_{G} \times \operatorname{Bun}_{G}$.

On the left-hand side of the categorical Langlands correspondence, the kernel corresponding to $\mathbb{W}_{d, \rho}$ is just $\Delta_{*}\left(\mathcal{F}_{d, \rho}\right)$, supported on the diagonal in $\operatorname{Loc}_{L_{G}} \times \operatorname{Loc}_{L_{G}}$.

Thus, we find that the categorical version of the geometric Langlands correspondence should yield the isomorphism,

$$
\operatorname{RHom}\left(\Delta_{*}(\mathcal{O}), \Delta_{*}\left(\mathcal{F}_{d, \rho}\right)\right) \simeq \operatorname{RHom}\left(\Delta_{!}(\underline{\mathcal{C}}), \overline{\mathcal{K}}_{d, \rho}\right) .
$$

By adjunction, the right-hand side of (6.6) is isomorphic to

$$
H^{\bullet}\left(\operatorname{Bun}_{G}, \Delta ! \overline{\mathcal{K}}\right)=H^{\bullet}\left(\operatorname{Bun}_{G}, \Delta ! \mathbf{p}_{*}(\mathcal{K})\right)=H^{\bullet}\left(\mathcal{M}_{d}, \Delta ! \mathcal{H}^{\prime}\left(\mathcal{K}_{d, \rho}\right)\right)
$$

(see formula (5.13) ). The last space is the vector space (5.14) that we proposed as a geometrization of the orbital side of the trace formula!

Hence the left-hand side of (6.6) should be the sought-after geometrization of the spectral side of the trace formula. Let us rewrite it using adjunction as follows:

$$
\operatorname{RHom}\left(\Delta^{*} \Delta_{*}(\mathcal{O}), \mathcal{F}_{d, \rho}\right) .
$$

Then we obtain the following isomorphism, which we conjecture as a geometric trace formula.

Conjecture 6. There is an isomorphism of vector spaces,

$$
\operatorname{RHom}\left(\Delta^{*} \Delta_{*}(\mathcal{O}), \mathcal{F}_{d, \rho}\right) \simeq H^{\bullet}\left(\operatorname{Bun}_{G}, \Delta^{!}\left(\overline{\mathcal{K}}_{d, \rho}\right)\right) .
$$

Thus, starting with the categorical Langlands correspondence, we have arrived at what we propose as a geometrization of the trace formula.

We have a similar conjecture for more general functors $\mathbb{K}$ which are compositions of $\mathbb{K}_{d, \rho}$ and Hecke functors at finitely many points of $X$ (see $[\mathrm{FN}]$ ). 
6.4. Connection to the Atiyah-Bott-Lefschetz fixed point formula. In [FN] we gave the following heuristic explanation why we should think of the space (6.7) as a geometrization of the sum appearing on the left-hand side of (6.2).

Let $\mathcal{F}$ be a coherent sheaf on $\operatorname{Loc}_{L_{G}}$ built from the vector bundles $\mathcal{T}_{\rho, x}, x \in|X|$ (like $\mathcal{F}_{d, \rho}$ constructed below). We would like to interpret taking the trace of the Frobenius on the (coherent!) cohomology $H^{\bullet}\left(\operatorname{Loc}_{L_{G}}, \mathcal{F}\right)$ as the sum over points $\sigma$ of $\operatorname{Loc}_{L_{G}}$, which we think of as the fixed points of the Frobenius automorphism acting on a moduli of homomorphisms

$$
\pi_{1}(X \underset{k}{\otimes} \bar{k}) \rightarrow{ }^{L} G
$$

Recall the Atiyah-Bott-Lefshetz fixed point formula [ $\mathrm{AB}$ (see also [1] §6]). Let $M$ be a smooth proper scheme, $V$ a vector bundle on $M$, and $V$ the coherent sheaf of sections of $V$. Let $u$ be an automorphism acting on $M$ with isolated fixed points, and suppose that we have an isomorphism $\gamma: u^{*}(\mathcal{V}) \simeq \mathcal{V}$. Then

$$
\operatorname{Tr}\left(\gamma, H^{\bullet}(M, \mathcal{V})\right)=\sum_{p \in M^{u}} \frac{\operatorname{Tr}\left(\gamma, \mathcal{V}_{p}\right)}{\operatorname{det}\left(1-\gamma, T_{p}^{*} M\right)},
$$

where

$$
M^{u}=\Gamma_{u} \underset{M \times M}{\times} \Delta
$$

is the set of fixed points of $u$, the fiber product of the graph $\Gamma_{u}$ of $u$ and the diagonal $\Delta$ in $M \times M$, which we assume to be transversal to each other (as always, the lefthand side of (6.8) stands for the alternating sum of traces on the cohomologies).

Now, if we take the cohomology not of $\mathcal{V}$, but of the tensor product $\mathcal{V} \otimes \Omega^{\bullet}(M)$, where $\Omega^{\bullet}(M)=\Lambda^{\bullet}\left(T^{*}(M)\right)$ is the graded space of differential forms, then the determinants in the denominators on the right-hand side of formula (6.8) will get canceled, and we will obtain the following formula:

$$
\operatorname{Tr}\left(\gamma, H^{\bullet}\left(M, \mathcal{V} \otimes \Omega^{\bullet}(M)\right)\right)=\sum_{p \in M^{u}} \operatorname{Tr}\left(\gamma, \mathcal{V}_{p}\right) .
$$

We would like to apply formula (6.9) in our situation. However, $\operatorname{Loc}_{L_{G}}$ is not a scheme, but an algebraic stack (unless ${ }^{L} G$ is a torus), so we need an analogue of (6.9) for algebraic stacks (and more generally, for derived algebraic stacks, since $\operatorname{Loc}_{L_{G}}$ is not smooth as an ordinary stack).

Let $M$ be as above and $\Delta: M \hookrightarrow M^{2}$ the diagonal embedding. Observe that

$$
\Delta^{*} \Delta_{*}(\mathcal{V}) \simeq \mathcal{V} \otimes \Omega^{\bullet}(M),
$$

and hence we can rewrite (6.9) as follows:

$$
\operatorname{Tr}\left(\gamma, H^{\bullet}\left(M, \Delta^{*} \Delta_{*}(\mathcal{V})\right)\right)=\sum_{p \in M^{u}} \operatorname{Tr}\left(\gamma, \mathcal{V}_{p}\right) .
$$

Now we propose formula (6.11) as a conjectural generalization of the AtiyahBott-Lefschetz fixed point formula to the case that $M$ is a smooth algebraic stack, and more generally, smooth derived algebraic stack (provided that both sides are well defined). We may also allow here $\mathcal{V}$ to be a perfect complex (as in II]).

\footnotetext{
${ }^{5}$ It is a great pleasure to do this here, because all of them were AMS Colloquium Lecturers in the past.
} 
Even more generally, we drop the assumption that $u$ has fixed points (or that the graph $\Gamma_{u}$ of $u$ and the diagonal $\Delta$ are transversal) and conjecture the following general fixed point formula of Atiyah-Bott type for (derived) algebraic stacks.

\section{Conjecture 7.}

$$
\operatorname{Tr}\left(\gamma, H^{\bullet}\left(M, \Delta^{*} \Delta_{*}(\mathcal{V})\right)\right)=\operatorname{Tr}\left(\gamma, H^{\bullet}\left(M^{u}, i_{u}^{*}(\mathcal{V})\right)\right),
$$

where $M^{u}=\Gamma_{u} \underset{M \times M}{\times} \Delta$ is the fixed locus of $u$, in the derived sense, and $i_{u}: M^{u} \rightarrow M$.

It is possible that Conjecture 7 may be proved using the methods of [BFN, $\mathrm{BN}] 6$

We want to apply (6.11) to the left-hand side of (6.7), which is

$$
\operatorname{RHom}\left(\Delta^{*} \Delta_{*}(\mathcal{O}), \mathcal{F}\right),
$$

where $\mathcal{F}=\mathcal{F}_{d, \rho}$. This is not exactly in the form of the left-hand side of (6.11), but it is very close. Indeed, if $M$ is a smooth scheme, then

$$
\operatorname{RHom}\left(\Delta^{*} \Delta_{*}(\mathcal{O}), \mathcal{F}\right) \simeq \mathcal{F} \otimes \Lambda^{\bullet}(T M),
$$

so we obtain the exterior algebra of the tangent bundle instead of the exterior algebra of the cotangent bundle. In our setting, we want $\gamma$ to be the Frobenius, and so a fixed point is a homomorphism $\pi_{1}(X) \rightarrow{ }^{L} G$. The tangent space to $\sigma$ (in the derived sense) should then be identified with the cohomology $H^{\bullet}(X$, ad $\circ \sigma)[1]$, and the cotangent space with its dual. Hence the trace of the Frobenius on the exterior algebra of the tangent space at $\sigma$ is the $L$-function $L(\sigma$, ad, $s)$ evaluated at $s=0$. Poincaré duality implies that the trace of the Frobenius on the exterior algebra of the cotangent space at $\sigma$ is

$$
L(\sigma, \operatorname{ad}, 1)=q^{-d_{G}} L(\sigma, \operatorname{ad}, 0),
$$

so the ratio between the traces on the exterior algebras of the tangent and cotangent bundles at the fixed points results in an overall factor which is a power of $q$ (which is due to our choice of conventions).

Hence, by following this argument and switching from $\mathbb{C}$ to $\mathbb{F}_{q}$, we obtain (up to a power of $q$ ) the trace formula (6.2) from the isomorphism (6.7).

The isomorphism (6.7) is still tentative, because there are some unresolved issues in the definition of the two sides (see $[\mathrm{FN}]$ for more detail). Nevertheless, we hope that further study of (6.7) will help us to gain useful insights into the trace formula and functoriality. I refer the reader to [FN], where in particular the abelian example is worked out and possible applications are discussed in the general case.

\section{Relative GeOmetric trace Formula}

In the previous section we discussed a geometrization of the trace formula (1.1). It appears in the framework of the categorical Langlands correspondence as the statement that the RHom's of kernels of certain natural functors are isomorphic. These kernels are sheaves on algebraic stacks over the squares $\operatorname{Bun}_{G} \times \operatorname{Bun}_{G}$ and $\operatorname{Loc}_{L_{G}} \times \operatorname{Loc}_{L_{G}}$.

It is natural to ask what kind of statement we may obtain if we consider instead the RHom's of sheaves on the stacks $\operatorname{Bun}_{G}$ and $\operatorname{Loc}_{L_{G}}$ themselves.

\footnotetext{
${ }^{6}$ After these notes were posted on the arXiv, we were informed by A. Polishchuk that our conjecture could be proved using the methods of $[\mathrm{P}]$. We thank Polishchuk for a useful discussion, which helped us to correct an inaccuracy in formula (6.12).
} 
In this section we will show, following closely [FN], that this way we obtain what may be viewed as a geometric analogue of the so-called relative trace formula. On the spectral side of this formula we also have a sum like (6.1), but with one important modification; namely, the eigenvalues $N_{\sigma}$ are weighted with the factor $L(\sigma, \operatorname{ad}, 1)^{-1}$. The insertion of this factor was originally suggested by Sarnak in $\left[\right.$ S and further studied by Venkatesh Ve, for the group $G L_{2}$ in the number field context. The advantage of this formula is that the summation is expected to be only over tempered representations and we remove the multiplicity factors $m_{\sigma}$.

7.1. Relative trace formula. We recall the setup of the relative trace formula.

Let $G$ be a split simple algebraic group over $k=\mathbb{F}_{q}$. In order to state the relative trace formula, we need to choose a non-degenerate character of $N(F) \backslash N\left(\mathbb{A}_{F}\right)$, where $N$ is a maximal unipotent subgroup of $G$. A convenient way to define it is to consider a twist of the group $G$. Let us pick a maximal torus $T$ such that $B=T N$ is a Borel subgroup. If the maximal torus $T$ admits the cocharacter $\check{\rho}: \mathbb{G}_{m} \rightarrow T$ equal to half-sum of all positive roots (corresponding to $B$ ), then let $K_{X}^{\check{\rho}}$ be the $T$-bundle on our curve $X$ which is the pushout of the $\mathbb{G}_{m}$-bundle $K_{X}^{\times}$(the canonical line bundle on $X$ without the zero section) under $\check{\rho}$. If $\check{\rho}$ is not a cocharacter of $T$, then its square is, and hence this $T$-bundle is well defined for each choice of the square root $K_{X}^{1 / 2}$ of $K_{X}$. We will make that choice once and for all 7

Now set

$$
G^{K}=K_{X}^{\check{\rho}} \underset{T}{\times} G, \quad N^{K}=K_{X}^{\check{\rho}} \underset{T}{\times} N,
$$

where $T$ acts via the adjoint action. For instance, if $G=G L_{n}$, then $G L_{n}^{K}$ is the group scheme of automorphism of the rank $n$ bundle $\mathcal{O} \oplus K_{X} \oplus \ldots \oplus K_{X}^{\otimes(n-1)}$ on $X$ (rather than the trivial bundle $\mathcal{O}^{\oplus n}$ ).

We have

$$
N^{K} /\left[N^{K}, N^{K}\right]=K_{X}^{\oplus \operatorname{rank}(G)} .
$$

Now let $\psi: \mathbb{F}_{q} \rightarrow \mathbb{C}^{\times}$be an additive character, and define a character $\Psi$ of $N^{K}\left(\mathbb{A}_{F}\right)$ as follows

$$
\Psi\left(\left(u_{x}\right)_{x \in|X|}\right)=\prod_{x \in|X|} \prod_{i=1}^{\operatorname{rank} G} \psi\left(\operatorname{Tr}_{k_{x} / k} \operatorname{Res}_{x}\left(u_{x, i}\right)\right),
$$

where $u_{x, i} \in K_{X}\left(F_{x}\right)$ is the $i$ th projection of $u_{x} \in N^{K}\left(F_{x}\right)$ onto $K_{X}\left(F_{x}\right)$ via the isomorphism (7.1). We denote by $k_{x}$ the residue field of $x$, which is a finite extension of the ground field $k=\mathbb{F}_{q}$.

By the residue formula, $\Psi$ is trivial on the subgroup $N^{K}(F)$ (this was the reason why we introduced the twist). It is also trivial on $N^{K}(\mathcal{O})$.

In what follows, in order to simplify notation, we will denote $G^{K}$ and $N^{K}$ simply by $G$ and $N$.

Given an automorphic representation $\pi$ of $G\left(\mathbb{A}_{F}\right)$, we have the Whittaker functional $W: \pi \rightarrow \mathbb{C}$,

$$
W(f)=\int_{N(F) \backslash N\left(\mathbb{A}_{F}\right)} f(u) \Psi^{-1}(u) d u,
$$

where $d u$ is the Haar measure on $N\left(\mathbb{A}_{F}\right)$ normalized so that the volume of $N\left(\mathcal{O}_{F}\right)$ is equal to 1 .

\footnotetext{
${ }^{7}$ This choice is related to the ambiguity of the equivalence (3.10); see footnote 2 on page 19
} 
We choose, for each unramified automorphic representation $\pi$, a non-zero $G\left(\mathcal{O}_{F}\right)$ invariant function $f_{\pi} \in \pi$ on $G(F) \backslash G\left(\mathbb{A}_{F}\right)$.

Let $K$ again be a kernel on the square of $\operatorname{Bun}_{G}(k)=G(F) \backslash G\left(\mathbb{A}_{F}\right) / G\left(\mathcal{O}_{F}\right)$ and $\mathbf{K}$ the corresponding integral operator acting on unramified automorphic functions. The simplest unramified version of the relative trace formula reads (here we restrict the summation to cuspidal automorphic representations $\pi$ )

$$
\begin{aligned}
\sum_{\pi} \overline{W_{\Psi}\left(f_{\pi}\right)} W_{\Psi}\left(K \cdot f_{\pi}\right)\left\|f_{\pi}\right\|^{-2} & \int_{N(F) \backslash N\left(\mathbb{A}_{F}\right)} \int_{N(F) \backslash N\left(\mathbb{A}_{F}\right)} K\left(u_{1}, u_{2}\right) \Psi^{-1}\left(u_{1}\right) \Psi\left(u_{2}\right) d u_{1} d u_{2}
\end{aligned}
$$

(see, e.g., [J]), where

$$
\|f\|^{2}=\int_{G(F) \backslash G\left(\mathbb{A}_{F}\right)}|f(g)|^{2} d g,
$$

and $d g$ denotes the invariant Haar measure normalized so that the volume of $G\left(\mathcal{O}_{F}\right)$ is equal to 1 . Note that

$$
\|f\|^{2}=q^{d_{G}} L(G)\|f\|_{T}^{2},
$$

where $\|f\|_{T}^{2}$ is the norm corresponding to the Tamagawa measure, $d_{G}=$ $(g-1) \operatorname{dim} G$,

$$
L(G)=\prod_{i=1}^{\ell} \zeta\left(m_{i}+1\right),
$$

where the $m_{i}$ are the exponents of $G$.

The following conjecture was communicated to us by B. Gross and A. Ichino. In the case of $G=\mathrm{SL}_{n}$ or $\mathrm{PGL}_{n}$, formula (7.3) follows from the Rankin-Selberg convolution formulas (see [FN]). Other cases have been considered in [GP, Ic, IcIk]. For a general semi-simple group $G$ of adjoint type formula (7.3) has been conjectured by $\mathrm{A}$. Ichino and T. Ikeda assuming that $\pi$ is square integrable. Note that if a square-integrable representation is tempered, then it is expected to be cuspidal, and that is why formula (17.3) is stated only for cuspidal representations.

Recall that an $L$-packet of automorphic representations is called generic if each irreducible representation $\pi=\bigotimes^{\prime} \pi_{x}$ from this $L$-packet has the property that the local $L$-packet of $\pi_{x}$ contains a generic representation (with respect to a particular choice of non-degenerate character of $N\left(F_{x}\right)$ ).

Conjecture 8. Suppose that the L-packet corresponding to an unramified $\sigma$ : $W_{F} \rightarrow{ }^{L} G$ is generic. Then it contains a unique, up to an isomorphism, irreducible representation $\pi$ such that $W_{\Psi}\left(f_{\pi}\right) \neq 0$, with multiplicity $m_{\pi}=1$. Moreover, if this $\pi$ is in addition cuspidal, then the following formula holds:

$$
\left|W_{\Psi}\left(f_{\pi}\right)\right|^{2}\left\|f_{\pi}\right\|^{-2}=q^{d_{N}-d_{G}} L(\sigma, \operatorname{ad}, 1)^{-1}\left|S_{\sigma}\right|^{-1},
$$

where $S_{\sigma}$ is the (finite) centralizer of the image of $\sigma$ in ${ }^{L} G$,

$$
d_{N}=-(g-1)(4\langle\rho, \check{\rho}\rangle-\operatorname{dim} N)
$$

(see formula (7.6) ), and $d_{G}=(g-1) \operatorname{dim} G$. 
Furthermore, we expect that if the $L$-packet corresponding to $\sigma: W_{F} \times \mathrm{SL}_{2} \rightarrow{ }^{L} G$ is non-generic, then

$$
L(\sigma, \operatorname{ad}, 1)^{-1}=0 .
$$

Ichino has explained to us that according to Arthur's conjectures, square-integrable non-tempered representations are non-generic.

Recall that we have $\mathbf{K} \cdot f_{\pi}=N_{\sigma} f_{\pi}$. Therefore Conjecture 8 and formula (7.2) give us the following:

$$
\begin{aligned}
q^{-d_{G}} \sum_{\sigma: W_{F} \rightarrow{ }^{L} G} N_{\sigma} \cdot L(\sigma, \mathrm{ad}, 1)^{-1}\left|S_{\sigma}\right|^{-1} \\
=q^{-d_{N}} \iint K\left(u_{1}, u_{2}\right) \Psi^{-1}\left(u_{1}\right) \Psi\left(u_{2}\right) d u_{1} d u_{2} .
\end{aligned}
$$

On the left-hand side we sum only over unramified $\sigma$, and only those of them contribute for which the corresponding $L$-packet of automorphic representations $\pi$ is generic.

We expect that the left-hand side of formula (17.5) has the following properties:

(1) It does not include homomorphisms $\sigma: \mathrm{SL}_{2} \times W_{F} \rightarrow{ }^{L} G$ which are nontrivial on the Artur's $\mathrm{SL}_{2}$.

(2) The multiplicity factor $m_{\sigma}$ of formula (6.2) disappears, because only one irreducible representation from the $L$-packet corresponding to $\sigma$ shows up (with multiplicity one).

(3) Since $S_{\sigma}$ is the group of automorphisms of $\sigma$, the factor $\left|S_{\sigma}\right|^{-1}$ makes the sum on the left-hand side (7.5) look like the Lefschetz fixed point formula for stacks.

7.2. Geometric meaning: right-hand side. Now we discuss the geometric meaning of formula (7.5), starting with the right-hand side. Let $\operatorname{Bun}_{N}^{\mathcal{F}_{T}}$ be the moduli stack of $B=B^{K}$ bundles on $X$ such that the corresponding $T$-bundle is $\mathcal{F}_{T}=K_{X}^{\check{\rho}}$. Note that

$$
\operatorname{dim} \operatorname{Bun}_{N}^{\mathcal{F}_{T}}=d_{N}=-(g-1)(4\langle\rho, \check{\rho}\rangle-\operatorname{dim} N) .
$$

Let ev : $\operatorname{Bun}_{N}^{\mathcal{F}_{T}} \rightarrow \mathbb{G}_{a}$ be the map constructed in FGV1.

For instance, if $G=G L_{2}$, then $\operatorname{Bun}_{N}^{\mathcal{F}_{T}}$ classifies rank-two vector bundles $\mathcal{V}$ on $X$ which fit in the exact sequence

$$
0 \rightarrow K_{X}^{1 / 2} \rightarrow \mathcal{V} \rightarrow K_{X}^{-1 / 2} \rightarrow 0
$$

where $K_{X}^{1 / 2}$ is a square root of $K_{X}$ which we have fixed. The map ev assigns to such $\mathcal{V}$ its extension class in $\operatorname{Ext}\left(\mathcal{O}_{X}, K_{X}\right)=H^{1}\left(X, K_{X}\right) \simeq \mathbb{G}_{a}$. For other groups the construction is similar (see FGV1).

On $\mathbb{G}_{a}$ we have the Artin-Schreier sheaf $\mathcal{L}_{\psi}$ associated to the additive character $\psi$. We define the sheaf

$$
\widetilde{\Psi}=\operatorname{ev}^{*}\left(\left.\mathcal{L}\right|_{\psi}\right)
$$

on $\operatorname{Bun}_{N}^{\mathcal{F}_{T}}$. Next, let $p: \operatorname{Bun}_{N}^{\mathcal{F}_{T}} \rightarrow \operatorname{Bun}_{G}$ be the natural morphism. Let

$$
\Psi=p_{!}(\widetilde{\Psi})\left[d_{N}-d_{G}\right]\left(\left(d_{N}-d_{G}\right) / 2\right) .
$$


Then the right-hand side of (7.5) is equal to the trace of the Frobenius on the vector space

$$
\operatorname{RHom}\left(\Psi, \mathbb{K}_{d, \rho}(\Psi)\right) .
$$

Here we use the fact that $\mathbb{D} \circ \mathbb{K} \simeq \mathbb{K} \circ \mathbb{D}$ and $\mathbb{D}(\widetilde{\Psi}) \simeq \operatorname{ev}^{*}\left(\left.\mathcal{L}\right|_{\psi^{-1}}\right)\left[2 d_{N}\right]\left(d_{N}\right)$.

7.3. Geometric meaning: left-hand side. As discussed above, we do not have an algebraic stack parametrizing homomorphisms $\sigma: W_{F} \rightarrow{ }^{L} G$ if our curve $X$ is defined over a finite field $\mathbb{F}_{q}$. But such a stack exists when $X$ is over $\mathbb{C}$, though in this case there is no Frobenius operator on the cohomology whose trace would yield the desired number (the left-hand side of (7.5)). In this subsection we will define a certain vector space (when $X$ is over $\mathbb{C}$ ) and conjecture that it is isomorphic to the vector space (7.8) which is the geometrization of the right-hand side of (7.5) (and which is well defined for $X$ over both $\mathbb{F}_{q}$ and $\mathbb{C}$ ). This will be our relative geometric trace formula. In the next subsection we will show that this isomorphism is a corollary of the categorical version of the geometric Langlands correspondence.

In order to define this vector space, we will use the coherent sheaf $\mathcal{F}_{d, \rho}$ on $\operatorname{Loc}_{L_{G}}$ introduced in Section 6.3. We propose that the geometrization of the left-hand side of (17.5) in the case when $\mathbb{K}=\mathbb{K}_{d, \rho}$ is the cohomology

$$
H^{\bullet}\left(\operatorname{Loc}_{L}, \mathcal{F}_{d, \rho}\right) .
$$

The heuristic explanation for this proceeds along the lines of the explanation given in the case of the ordinary trace formula in Section 6.4, using the AtiyahBott-Lefschetz fixed point formula 8 We wish to apply it to the cohomology (7.9). If $\operatorname{Loc}_{L_{G}}$ were a smooth scheme, then we would have to multiply the number $N_{\sigma}$ which corresponds to the stalk of $\mathcal{F}_{d, \rho}$ at $\sigma$, by the factor

$$
\operatorname{det}\left(1-\mathrm{Fr}, T_{\sigma}^{*} \operatorname{Loc}_{L}\right)^{-1} \text {. }
$$

Recall that the tangent space to $\sigma$ (in the derived sense) may be identified with the cohomology $H^{\bullet}(X$, ad $\circ \sigma)[1]$. Using the Poincaré duality, we find that the factor (7.10) is equal to

$$
L(\sigma, \operatorname{ad}, 1)^{-1} .
$$

Therefore, if we could apply the Lefschetz fixed point formula to the cohomology (7.9) and write it as a sum over all $\sigma: W_{F} \rightarrow{ }^{L} G$, then the result would be the left-hand side of (7.5) (up to a factor that is a power of $q$ ). (Note however that since $\operatorname{Loc}_{L_{G}}$ is not a scheme, but an algebraic stack, the weighting factor should be more complicated for those $\sigma$ which admit non-trivial automorphisms; see the conjectural fixed point formula (6.12) in Section 6.4.)

This leads us to the following relative geometric trace formula (in the case of the functor $\mathbb{K}_{d, \rho}$ ).

Conjecture 9. We have the following isomorphism of vector spaces:

$$
H^{\bullet}\left(\operatorname{Loc}_{L_{G}}, \mathcal{F}_{d, \rho}\right) \simeq \mathrm{RHom}_{\mathrm{Bun}_{\mathrm{G}}}\left(\Psi, \mathbb{K}_{d, \rho}(\Psi)\right) .
$$

Now we explain how the isomorphism (7.11) fits in the framework of a categorical version of the geometric Langlands correspondence.

\footnotetext{
${ }^{8}$ We note that applications of the Atiyah-Bott-Lefschetz fixed point formula in the context of Galois representations have been previously considered by M. Kontsevich in $\mathrm{K}$.
} 
7.4. Interpretation from the point of view of the categorical Langlands correspondence. We start by asking what is the $\mathcal{D}$-module on $\operatorname{Bun}_{G}$ corresponding to the structure sheaf $\mathcal{O}$ on $\operatorname{Loc}_{L_{G}}$ under the categorical Langlands correspondence of Section 3.7. The following answer was suggested by Drinfeld (see [VLaf]): it is the sheaf $\Psi$ that we have discussed above.

The rationale for this proposal is the following: We have

$$
\operatorname{RHom}_{\operatorname{Loc}_{L_{G}}}\left(\mathcal{O}, \mathcal{O}_{\sigma}\right)=\mathbb{C}, \quad \forall \sigma,
$$

where $\mathcal{O}_{\sigma}$ is again the skyscraper sheaf supported at $\sigma$. Therefore, since $C\left(\mathcal{O}_{\sigma}\right)=$ $\mathcal{F}_{\sigma}$, we should have, according to (6.4),

$$
\mathrm{RHom}_{\mathrm{Bun}_{G}}\left(C(\mathcal{O}), \mathcal{F}_{\sigma}\right)=\mathbb{C}, \quad \forall \sigma .
$$

According to the conjecture of [LafL, the sheaf $\Psi$ has just this property:

$$
\operatorname{RHom}_{\mathrm{Bun}_{G}}\left(\Psi, \mathcal{F}_{\sigma}\right)
$$

is the one-dimensional vector space in cohomological degree 0 (if we use appropriate normalization for $\mathcal{F}_{\sigma}$ ).

This vector space should be viewed as a geometrization of the Fourier coefficient of the automorphic function corresponding to $\mathcal{F}_{\sigma}$.

This provides some justification for the assertion that 9

$$
C(\mathcal{O})=\Psi
$$

Next, we rewrite (17.9) as

$$
\operatorname{RHom}_{\operatorname{Loc}_{L_{G}}}\left(\mathcal{O}, \mathbb{W}_{d, \rho}(\mathcal{O})\right) \text {. }
$$

Using the compatibility (3.12) of $C$ with the Wilson/Hecke operators and formulas (6.4) and (7.12), we obtain that (7.13) should be isomorphic to

$$
\operatorname{RHom}_{\mathrm{Bun}_{G}}\left(\Psi, \mathbb{K}_{d, \rho}(\Psi)\right),
$$

which is the right-hand side of (7.11).

Thus, we obtain that the relative geometric trace formula (7.11) follows from the categorical version of the geometric Langlands correspondence. We hope that this formula may also be applied to the Functoriality Conjecture.

\section{ACKNOWLEDGMENTS}

The author is grateful to Robert Langlands and Ngô Bao Châu for their collaboration on the papers [FLN, [FN] which are reviewed in these notes.

The author also thanks Ivan Fesenko for his comments on a draft of this paper.

\section{ABOUt THE AUTHOR}

Edward Frenkel is professor of mathematics at University of California, Berkeley. He has written two books, most recently, Langlands Correspondence for Loop Groups, and is now working on a third about the Langlands Program and Quantum Field Theory. He was awarded the Hermann Weyl Prize in 2002. In recent years, Frenkel turned to cinema and writing in order to convey the beauty of mathematics to the wide audience. His book Love and Math will be published by Basic Books in October 2013. In January 2012 he delivered the AMS Colloquium Lectures at the Joint Mathematics Meetings in Boston. This article is based on these lectures.

\footnotetext{
${ }^{9}$ As explained in footnote 2 on page 19 corresponds to a particular choice of $K_{X}^{1 / 2}$. Given such a choice, $C(\mathcal{O})$ should be the character sheaf $\Psi$ associated to that $K_{X}^{1 / 2}$.
} 


\section{REFERENCES}

[AG] D. Arinkin and D. Gaitsgory, Singular support of coherent sheaves, and the geometric Langlands conjecture, Preprint arXiv:1201.6343.

[Art1] J. Arthur, The principle of functoriality, Bull. Amer. Math. Soc. 40 (2002) 39-53.

[Art2] J. Arthur, An Introduction to the trace formula, Clay Mathematics Proceedings 4, American Mathematical Society, Providence, RI, 2005.

[AB] M. Atiyah and R. Bott, A Lefschetz fixed point formula for elliptic complexes, I. Ann. of Math. (2) 86 (1967) 374-407; and II. Ann. of Math. (2) 881968 451-491.

[BCDT] C. Breuil, B. Conrad, F. Diamond and R. Taylor, On the modularity of elliptic curves over Q: wild 3-adic exercises, J. Amer. Math. Soc. 14 (2001) 843-939.

[BL] A. Beauville and Y. Laszlo, Un lemme de descente, C.R. Acad. Sci. Paris, Sér. I Math. 320 (1995) 335-340.

[Be] K. Behrend, Derived l-adic categories for algebraic stacks, Mem. Amer. Math. Soc. 163 (2003), no. 774 .

[BeDh] K. Behrend and A. Dhillon, Connected components of moduli stacks of torsors via Tamagawa numbers, Canad. J. Math. 61 (2009) 3-28.

[BFN] D. Ben-Zvi, J. Francis, and D. Nadler, Integral transforms and Drinfeld centers in derived algebraic geometry, Preprint arXiv:0805.0157, to appear in Journal of AMS.

[BN] D. Ben-Zvi and D. Nadler, Loop spaces and connections, Preprint arXiv:1002.3636.

[BD] A. Beilinson and V. Drinfeld, Quantization of Hitchin's integrable system and Hecke eigensheaves, Preprint, available at www.math.uchicago.edu/ mitya/langlands

[Bou] N. Bourbaki, Groupes et algèbres de Lie Chapitres IV, V, VI, Hermann, Paris 1968.

[CL] P.-H. Chaudouard and G. Laumon, Le lemme fondamental pondéré I : constructions géométriques, Preprint arXiv:0902.2684.

[D1] V.G. Drinfeld, Two-dimensional $\ell$-adic representations of the fundamental group of a curve over a finite field and automorphic forms on GL(2), Amer. J. Math. 105 (1983) $85-114$

[D2] V.G. Drinfeld, Langlands conjecture for $G L(2)$ over function field, Proc. Int. Congress of Math. (Helsinki, 1978), pp. 565-574; Moduli varieties of F-sheaves, Funct. Anal. Appl. 21 (1987) 107-122; The proof of Petersson's conjecture for $G L(2)$ over a global field of characteristic p, Funct. Anal. Appl. 22 (1988) 28-43.

[F1] E. Frenkel, "Lectures on the Langlands Program and Conformal Field Theory", in Frontiers in Number Theory, Physics and Geometry II, eds. P. Cartier, et al., pp. 387-536, Springer Verlag, 2007 (hep-th/0512172).

[F2] E. Frenkel, Gauge theory and Langlands duality, Séminaire Bourbaki, Juin 2009 (arXiv:0906.2747).

[FGV1] E. Frenkel, D. Gaitsgory and K. Vilonen, Whittaker patterns in the geometry of moduli spaces of bundles on curves, Ann. of Math. 153 (2001) 699-748, (arXiv:math/9907133).

[FGV2] E. Frenkel, D. Gaitsgory and K. Vilonen, On the geometric Langlands conjecture, Jour. Amer. Math. Soc. 15 (2001) 367-417 (arXiv:math/0012255).

[FLN] E. Frenkel, R. Langlands and B.C. Ngô, La formule des traces et la functorialité. Le début d'un Programme, Ann. Sci. Math. Québec 34 (2010) 199-243 (arXiv:1003.4578).

[FN] E. Frenkel and B.C. Ngô, Geometrization of trace formulas, Bull. Math. Sci. 1 (2011) 1-71 (arXiv:1004.5323). MR2823791

[FW] E. Frenkel and E. Witten, Geometric endoscopy and mirror symmetry, Communications in Number Theory and Physics, 2 (2008) 113-283 (arXiv:0710.5939).

[FK] E. Freitag, R. Kiehl, Etale Cohomology and the Weil conjecture, Springer, 1988.

[G] D. Gaitsgory, On a vanishing conjecture appearing in the geometric Langlands correspondence, Ann. Math. 160 (2004) 617-682.

[GM] S.I. Gelfand and Yu.I. Manin, Homological Algebra, Encyclopedia of Mathematical Sciences 38, Springer, 1994. MR2417848 (2009e:14017)

[GP] B.H. Gross and D. Prasad, On the decomposition of a representation of $S_{n}$ when restricted to $\mathrm{SO}_{n-1}$, Canad. J. Math. 44 (1992) 974-1002.

[Ha] G. Harder. Über die Galoiskohomologie halbenfacher algebraischer Gruppen. III, J. Reine Angew. Math. 274/275 (1975) 125-138.

[H1] N. Hitchin, The self-duality equations on a Riemann surface, Proc. London Math. Soc. (3) 55 (1987) 59-126. 
[H2] N. Hitchin, Stable bundles and integrable systems, Duke Math. J. 54 (1987) 91-114.

[Il] L. Illusie, Formule de Lefschetz, SGA 5, Lect. Notes in Math. 589, pp. 73-137, Springer Verlag, 1977.

[Ic] A. Ichino, On critical values of adjoint L-functions for GSp(4), Preprint, available at http://www.math.ias.edu/ ichino/ad.pdf

[IcIk] A. Ichino and T. Ikeda, On the periods of automorphic forms on special orthogonal groups and the Gross-Prasad conjecture, Geom. Func. Anal. 19 (2010) 1378-1425.

[J] H. Jacquet, "A guide to the relative trace formula", in Automorphic Representations, Lfunctions and Applications: Progress and Prospects, Ohio State University Mathematical Research Institute Publications, Volume 11, pp. 257-272, De Gruyter, Berlin, 2005.

[JL] H. Jacquet and R. Langlands, Automorphic forms on GL(2), Lect. Notes in Math. 114, Springer, 1970.

$\mathrm{X}$

[KW] A. Kapustin and E. Witten, Electric-magnetic duality and the geometric Langlands Program, Preprint hep-th/0604151.

[KS] M. Kashiwara and P. Schapira, Sheaves on Manifolds, Springer, 1990.

[K] M. Kontsevich, Notes on motives in finite characteristic, Preprint arXiv:math/0702206.

[LLaf] L. Lafforgue, Chtoucas de Drinfeld et correspondance de Langlands, Invent. Math. 147 (2002) 1-241.

[VLaf] V. Lafforgue, Quelques calculs reliés à la correspondance de Langlands géométrique pour $\mathbb{P}^{1}$, available at http://people.math.jussieu.fr/ vlafforg/geom.pdf

[LL] J.-P. Labesse and R.P. Langlands, L-indistinguishability for SL(2), Canad. J. Math. 31 (1979) $726-785$.

[LafL] V. Lafforgue and S. Lysenko, Compatibility of the theta correspondence with the Whittaker functors, Preprint arXiv:0902.0051.

[L1] R. Langlands, Problems in the theory of automorphic forms, in Lect. Notes in Math. 170, pp. 18-61, Springer Verlag, 1970.

[L2] R. Langlands, "Beyond endoscopy", in Contributions to automorphic forms, geometry, and number theory, pp. 611-697, Johns Hopkins Univ. Press, Baltimore, MD, 2004.

[L3] R. Langlands, Un nouveau point de repère dans la théorie des formes automorphes, Canad. Math. Bull. 50 (2007) no. 2, 243-267.

[L4] E. Langlands, Singularités et Transfert, 2010, available at http://publications.ias.edu/rpl

[L5] E. Langlands, Functoriality and Reciprocity (in Russian), 2011, http://publications.ias.edu/rpl

[LO] Y. Laszlo and M. Olsson, The six operations for sheaves on Artin stacks. I. Finite coefficients, Publ. Math. IHES 107 (2008) 109-168.

[Lau1] G. Laumon, Transformation de Fourier, constantes d'équations fonctionelles et conjecture de Weil, Publ. IHES 65 (1987) 131-210.

[Lau2] G. Laumon, Transformation de Fourier généralisée, Preprint alg-geom/9603004.

[Lau3] G. Laumon, Correspondance de Langlands géométrique pour les corps de fonctions, Duke Math. J. 54 (1987) 309-359.

[Ly1] S. Lysenko, Geometric theta-lifting for the dual pair $S O_{2 m}, S p_{2 n}$, Ann. Sci. École Norm. Sup. 44 (2011) 427-493.

[Ly2] S. Lysenko, Geometric theta-lifting for the dual pair $G S p_{2 n}, G_{2 m}$, Preprint arXiv:0802.0457.

[M] J.S. Milne, Étale cohomology, Princeton University Press, 1980.

[MV] I. Mirković and K. Vilonen, Geometric Langlands duality and representations of algebraic groups over commutative rings, Preprint math.RT/0401222.

[N1] B.C. Ngô, Fibration de Hitchin et endoscopie, Invent. Math. 164 (2006) 399-453.

[N2] B.C. Ngô, Le lemme fondamental pour les algebres de Lie, Preprint arXiv:0801.0446.

[Ni1] Ye. Nisnevich, Étale cohomology and arithmetic of semisimple groups, Ph.D. Thesis. Harvard University, 1982. Available at http://proquest.umi.com/pqdlink?RQT=306

[Ni2] Ye. Nisnevich, Espaces homogènes principaux rationnellement triviaux et arithmétique des schémas en groupes réductifs sur les anneaux de Dedekind, C. R. Acad. Sci. Paris Sér. I Math. 299 (1984) 5-8.

[P] A. Polishchuk, Lefschetz type formulas for dg-categories, Preprint arXiv:1111.0728.

[R] M. Rothstein, Connections on the total Picard sheaf and the KP hierarchy, Acta Applicandae Mathematicae 42 (1996) 297-308. 
[S] P. Sarnak, Comments on Robert Langland's Lecture: "Endoscopy and Beyond", available at http://www.math.princeton.edu/sarnak/SarnakLectureNotes-1.pdf

[TW] R. Taylor and A. Wiles, Ring-theoretic properties of certain Hecke algebras, Ann. of Math. (2) 141 (1995) 553-572.

[Ve] A. Venkatesh, "Beyond endoscopy" and special forms on GL(2), J. Reine Angew. Math. 577 (2004) 23-80.

[Vi] E. B. Vinberg, On reductive algebraic semigroups, Lie Groups and Lie Algebras, in E. B. Dynkin Seminar (S. Gindikin, E. Vinberg, eds.), AMS Transl. (2) 169 (1995), 145-182.

[W] A. Wiles, Modular elliptic curves and Fermat's Last Theorem, Ann. of Math. (2) 141 (1995) 443-551.

Department of Mathematics, University of California, Berkeley, California 94720 